\title{
Semistrict higher gauge theory
}

\author{
Branislav Jurčo, ${ }^{a}$ Christian Sämann $^{b}$ and Martin Wolf ${ }^{c}$ \\ ${ }^{a}$ Mathematical Institute, Faculty of Mathematics and Physics, Charles University in Prague, \\ Prague 186 75, Czech Republic \\ ${ }^{b}$ Maxwell Institute for Mathematical Sciences, Department of Mathematics, \\ Heriot-Watt University, \\ Edinburgh EH14 4 AS, United Kingdom \\ ${ }^{c}$ Department of Mathematics, University of Surrey, \\ Guildford GU2 7XH, United Kingdom \\ E-mail: branislav.jurco@gmail.com, c.saemann@hw.ac.uk, \\ m.wolf@surrey.ac.uk
}

ABSTRACT: We develop semistrict higher gauge theory from first principles. In particular, we describe the differential Deligne cohomology underlying semistrict principal 2-bundles with connective structures. Principal 2-bundles are obtained in terms of weak 2-functors from the Cech groupoid to weak Lie 2-groups. As is demonstrated, some of these Lie 2-groups can be differentiated to semistrict Lie 2-algebras by a method due to Ševera. We further derive the full description of connective structures on semistrict principal 2bundles including the non-linear gauge transformations. As an application, we use a twistor construction to derive superconformal constraint equations in six dimensions for a nonAbelian $\mathcal{N}=(2,0)$ tensor multiplet taking values in a semistrict Lie 2-algebra.

KEYwords: Extended Supersymmetry, Differential and Algebraic Geometry, M-Theory, Integrable Field Theories

ARXIV EPRINT: 1403.7185 


\section{Contents}

1 Introduction, summary, and outlook 2

1.1 Motivation 2

1.2 Summary of results 4

1.3 Outlook 6

2 Preliminaries $\quad 7$

2.1 Weak 2-categories $\quad 7$

$\begin{array}{lll}2.2 & \text { Weak 2-groupoids } & 12\end{array}$

$\begin{array}{lll}2.3 & \text { Lie 2-groups } & 15\end{array}$

$\begin{array}{lll}2.4 & \text { Lie 2-algebras } & 17\end{array}$

3 Principal 2-bundles with Lie 2-groups 19

$\begin{array}{lll}3.1 & \text { Principal bundles as functors } & 20\end{array}$

3.2 Principal 2-bundles as 2-functors 21

$\begin{array}{lll}3.3 & \text { Semistrict and strict principal 2-bundles } & 26\end{array}$

4 Differentiating semistrict Lie 2-groups 28

$\begin{array}{lll}4.1 \text { Basic ideas } & 29\end{array}$

4.2 Lie algebra of a Lie group 31

4.3 Semistrict Lie 2-algebra of a semistrict Lie 2-group 33

4.4 Example: strict Lie 2-groups 41

4.5 Comment on differentiation and categorical equivalence 42

5 Semistrict higher gauge theory $\quad 43$

5.1 Local semistrict higher gauge theory 43

$\begin{array}{lll}5.2 & \text { Finite gauge transformations } & 45\end{array}$

$\begin{array}{lll}5.3 & \text { Connective structure } & 47\end{array}$

5.4 Semistrict non-Abelian Deligne cohomology 49

6 Application: Penrose-Ward transform $\quad \mathbf{5 2}$

$\begin{array}{lll}6.1 & \text { Supertwistor space } & 52\end{array}$

6.2 Penrose-Ward transform 54

$\begin{array}{lr}\text { A Strong homotopy Lie algebras } & 59\end{array}$

B Groupoid bundles $\quad 62$ 


\section{Introduction, summary, and outlook}

\subsection{Motivation}

Gauge theory is one of the most far-reaching concepts in modern theoretical physics as is exemplified by the impressive success of the standard model of elementary particles as well as many of the more recent developments in string theory such as the gauge/gravity correspondence. From a mathematical point of view, the kinematic data of classical gauge theory is described in terms of principal bundles with connection. Equivalence relations on this data, known as gauge transformations, are captured by a non-Abelian generalisation of the so-called first Abelian Deligne cohomology group.

By now, there is a well-established way of categorifying gauge theory to what is known as higher gauge theory. Here, the kinematic data lives on non-Abelian gerbes $[1,2]$ or the more general principal 2-bundles of Bartels [3]; higher categorifications leading to $p$-gerbes or principal $(p+1)$-bundles are also known, though explicit details are somewhat limited. The notion of a connection on principal bundles is generalised to so-called connective structures on principal 2-bundles. This is a well established approach albeit with one limitation: instead of featuring the most general, weak Lie 2-group as structure 2-group, the standard formulations employ so-called crossed modules of Lie groups, which are equivalent to strict Lie 2-groups.

The main aims of this paper are to lift this limitation and to discuss in full detail principal 2-bundles with connective structures that have semistrict Lie 2-groups as structure 2-groups. This involves considerably more technical effort than the strict case, and we would therefore like to give ample motivation for our goal.

The most general notion of a categorified group or 2-group which we shall consider here is what is usually called a weak 2 -group. Just as a group is a groupoid with a single object, a weak 2-group is a weak 2-groupoid with a single object. As shown by Baez \& Lauda [4], every weak 2-group can be enhanced to a coherent 2-group, and, furthermore, coherent 2-groups are categorically equivalent to strict 2-groups. Categorical equivalence, however, seems to be too coarse in many cases. Perhaps a prime example in this regard is the categorified operation of integrating a Lie 2-algebra: it is known that the string Lie 2-algebra cannot be integrated to a topological 2-group [4]. This semistrict Lie 2-algebra, however, is categorically equivalent to an infinite dimensional strict Lie 2-algebra, which can be integrated to a strict Lie 2-group [5]. Similarly, it is natural to expect that dynamical models of connective structures on principal 2-bundles will not necessarily agree even if the underlying structure 2-groups are categorically equivalent.

Our motivation for considering categorified differential geometry stems mostly from M-theory. Within M-theory, principal 2-bundles with connective structures arise quite naturally in a non-Abelian generalisation of the effective description of M5-branes. In particular, they capture the kinematic structure of the mysterious $\mathcal{N}=(2,0)$ superconformal field theory in six dimensions, or $(2,0)$-theory for short.

The existence of the $(2,0)$-theory has been shown by Witten [6] a long time ago. However, it remains unclear if this theory should have a classical description in terms of equations of motion or even a Lagrangian. Quite recently, there has been impressive success 
in the effective description of multiple M2-branes. Contrary to popular belief, it turned out that there are M2-brane models with a Lagrangian formulation, which pass many nontrivial consistency checks, see [7] for a review. Spurred by this success, various directions of research have been pursued to try to arrive at an analogous classical description for multiple M5-branes. In fact, much of the current research activities in string theory is devoted to a more detailed understanding of the $(2,0)$-theory.

Since the Abelian tensor multiplet in six dimensions contains a 2-form gauge potential described by a U(1)-gerbe, it is only natural to expect that the non-Abelian case is described by the connective structure of a principal 2-bundle. Principal 2-bundles with connective structures allow for the parallel transport of one-dimensional objects, which is certainly relevant in the description of the self-dual strings that form the boundaries of the M2branes mediating M5-brane interactions. A detailed explanation of the higher gauge theory approach to M5-branes can be found in Fiorenza, Sati \& Schreiber [8].

Besides its mathematical appeal, an important argument for the use of higher gauge theory is that principal 2-bundles can indeed yield superspace constraint equations for the $\mathcal{N}=(2,0)$ tensor multiplet in six dimensions. This was shown recently in Saemann \& Wolf $[9,10]$, and the derivation of these equations involved a description of the tensor multiplet in terms of certain holomorphic principal 2- and 3-bundles over a twistor space. Interestingly, such a twistorial description might also yield a Lagrangian formulation of the theory, as was already demonstrated for the Abelian case in [11, 12].

The constraint equations arising from a twistorial description starting from principal 2bundles with strict structure 2-groups turned out to be somewhat restrictive. A first reason for considering semistrict principal 2-bundles is therefore to generalise the superconformal constraint equations arising from a twistor description of the (2,0)-theory and we shall present the outcome in section 6. In particular, we shall see that semistrict principal 2bundles will allow for incorporating cubic terms in the connection 1-form in the definition of the 3-form curvature.

Another popular approach to deriving a classical description of the $(2,0)$-theory is based on a non-Abelian generalisation of the tensor hierarchy [13-17] with the closely related proposals of $[18,19]$. Here, one obtains $\mathcal{N}=(1,0)$ superconformal equations of motion as well as a Lagrangian description. These (1,0)-models have an underlying gauge algebraic structure which is strongly reminiscent of a semistrict Lie 3 -algebra. The detailed analysis of this algebraic structures in [20] showed that there is indeed a large overlap. Moreover, it was shown that certain classes of $(1,0)$-models are reformulations of higher gauge theories with strict Lie 3-groups. To fully compare the (1,0)-models with higher gauge theory, however, it is indispensable to develop a detailed description of gauge theory based on semistrict principal $n$-bundles. This is a second motivation for studying semistrict principal 2-bundles.

Further motivation for our study stems from the problem of differentiating semistrict Lie 2-groups to semistrict Lie 2-algebras. While there has been some effort to understand the integration of Lie 2-algebras to Lie 2-groups, see for example Getzler [21] and Henriques [22], the inverse operation does not seem to have attracted the same amount of attention. In the present work, we shall follow a general approach to this problem that 
was proposed by Ševera [23]. In this construction, one considers a simplicial manifold and extracts a corresponding $L_{\infty}$-algebra as its first jet. A Lie 2-group can be encoded in terms of a simplicial manifold as the so-called Duskin nerve of its delooping. The first jet of this simplicial manifold is then constructed as a functor acting on descent data of a trivial principal 2-bundle.

Finally, we would like to mention that a different proposal for semistrict higher gauge theory was given previously by Zucchini [24]. In this approach, the higher Maurer-Cartan forms are incorporated abstractly as constrained parameters into the gauge transformation. This is not the case in our approach; our detailed understanding of the differential cohomology underlying semistrict principal 2-bundles with connective structures makes the parameters of gauge transformations explicit.

\subsection{Summary of results}

For the reader's convenience, let us summarise our key results in an easily accessible way. In the following, we let $X$ be a smooth manifold with covering $\mathfrak{U}:=\left\{U_{a}\right\}$. Moreover, we let $\mathscr{G}=(M, N)$ be a weak Lie 2 -group, which can be equivalently regarded as a smooth weak 2 -groupoid with a single 0 -cell $e, \mathrm{~B} \mathscr{G}=(\{e\}, M, N)$. We denote the source and target maps by $\mathrm{s}$ and $\mathrm{t}$. Vertical and horizontal composition in this weak 2-groupoid are denoted by $\circ$ and $\otimes$, respectively, a stands for the associator and $I$ and $r$ label the left- and right-unitors.

A weak principal 2-bundle is described by a $\mathscr{G}$-valued Čech 2-cocycle. Such a cocycle is given by an $M$-valued Čech 1-cochain $\left\{m_{a b}\right\}$ together with an $N$-valued Čech 0 -cochain $\left\{n_{a}\right\}$ and an $N$-valued Čech 2-cochain $\left\{n_{a b c}\right\}$ which satisfy the following cocycle conditions, cf. Definition 3.8:

$$
\begin{aligned}
n_{a b c}: m_{a b} \otimes m_{b c} & \Rightarrow m_{a c} \\
n_{a c d} \circ\left(n_{a b c} \otimes \mathrm{id}_{m_{c d}}\right) & =n_{a b d} \circ\left(\operatorname{id}_{m_{a b}} \otimes n_{b c d}\right) \circ \mathrm{a}_{m_{a b}, m_{b c}, m_{c d}}
\end{aligned}
$$

and

$$
n_{a b b} \circ\left(\mathrm{id}_{m_{a b}} \otimes n_{b}\right)=\mathrm{I}_{m_{a b}} \quad \text { and } \quad n_{a a b} \circ\left(n_{a} \otimes \mathrm{id}_{m_{a b}}\right)=\mathrm{r}_{m_{a b}} .
$$

Two weak principal 2-bundles are called equivalent whenever their degree-2 Čech cocycles are related by a $\mathscr{G}$-valued Čech 2 -coboundary. This coboundary consists of an $M$ valued Čech 0-cochain $\left\{m_{a}\right\}$ and an $N$-valued Čech 1-cochain $\left\{n_{a b}\right\}$ such that for degree-2 Cech cocycles $\left(\left\{m_{a b}\right\},\left\{n_{a b c}\right\},\left\{n_{a}\right\}\right)$ and $\left(\left\{\tilde{m}_{a b}\right\},\left\{\tilde{n}_{a b c}\right\},\left\{\tilde{n}_{a}\right\}\right)$ the following holds, cf. Definition 3.10:

$$
\begin{aligned}
& n_{a b}: m_{a b} \otimes m_{b} \Rightarrow m_{a} \otimes \tilde{m}_{a b}, \\
& n_{a c} \circ\left(n_{a b c} \otimes \mathrm{id}_{m_{c}}\right)=\left(\mathrm{id}_{m_{a}} \otimes \tilde{n}_{a b c}\right) \circ \mathrm{a}_{m_{a}, \tilde{m}_{a b}, \tilde{m}_{b c}} \circ\left(n_{a b} \otimes \mathrm{id}_{\tilde{m}_{b c}}\right) \circ \\
& \circ \mathrm{a}_{m_{a b}, m_{b}, \tilde{m}_{b c}}^{-1} \circ\left(\operatorname{id}_{m_{a b}} \otimes n_{b c}\right) \circ \mathrm{a}_{m_{a b}, m_{b c}, m_{c}} \text {. }
\end{aligned}
$$

and

$$
n_{a a} \circ\left(n_{a} \otimes \mathrm{id}_{m_{a}}\right)=\left(\mathrm{id}_{m_{a}} \otimes \tilde{n}_{a}\right) \circ \mathrm{I}_{m_{a}}^{-1} \circ \mathrm{r}_{m_{a}} .
$$

As demonstrated in Proposition 3.15, every $\mathscr{G}$-valued Čech 2-cocycle is equivalent to a $\mathscr{G}$-valued Čech 2 -cocycle with all $\left\{n_{a}\right\}$ being trivial. 
Furthermore, we define semistrict Lie 2-groups $\mathscr{G}$ as weak Lie 2-groups in which leftand right-unitors as well as the unit and counit are all trivial. Following [23], we then consider a functor from the category of smooth manifolds to the category of $\mathscr{G}$-valued descent data on surjective submersions $\mathbb{R}^{0 \mid 1} \times X \rightarrow X$. This functor is parameterised by a 2 -term $L_{\infty}$-algebra as shown in Theorem 4.24 . This 2 -term $L_{\infty}$-algebra is, in turn, equivalent to the semistrict Lie 2 -algebra associated with the semistrict Lie 2 -group $\mathscr{G}$. Deriving the parametrisation of this functor is the higher equivalent of computing the Lie algebra of a Lie group.

Moreover, we demonstrate that local connective structures on principal 2-bundles with semistrict structure 2-group (as well as principal $n$-bundles with semistrict structure $n$ group) are readily derived. To this end, we consider the tensor product of the aforementioned 2-term $L_{\infty}$-algebra with the differential graded algebra of differential forms on $X$. This leads to another $L_{\infty}$-algebra as well as its homotopy Maurer-Cartan equation including infinitesimal gauge transformations as shown in Propositions 5.3 and 5.9.

The finite gauge transformations are derived from an equivalence relation among the functors considered in the above differentiation of a Lie 2-group $\mathscr{G}=(M, N)$ to a 2 -term $L_{\infty}$-algebra $\mathfrak{v} \stackrel{\mu_{1}}{\longrightarrow} \mathfrak{w}$ with $\mathfrak{w}:=T_{\mathrm{id}_{e}} M$ and $\mathfrak{v}:=\operatorname{ker}(\mathrm{t}) \subseteq T_{\mathrm{id}_{\mathrm{id}_{e}}} N$ and higher or homotopy products $\mu_{1,2,3}$. This relation is presented in Theorem 4.26, from which Proposition 5.9 can be gleaned: a connective structure over $U_{a} \subseteq X$ on a semistrict principal 2-bundle is given locally on a patch $U_{a}$ in terms of a $\mathfrak{w}$-valued differential 1-form $A_{a}$ and a $\mathfrak{v}$-valued differential 2-form $B_{a}$ such that the fake 2-form curvature

$$
\mathcal{F}_{a}:=\mathrm{d} A_{a}+A_{a} \otimes A_{a}+\mathrm{s}\left(B_{a}\right)
$$

vanishes. In addition, the curvature 3 -form $H_{a}$ is defined by

$$
H_{a}:=\mathrm{d} B_{a}+\mathrm{id}_{A_{a}} \otimes B_{a}-B_{a} \otimes \mathrm{id}_{A_{a}}+\mu\left(A_{a}, A_{a}, A_{a}\right),
$$

where $\mu\left(A_{a}, A_{a}, A_{a}\right): A_{a} \otimes\left(A_{a} \otimes A_{a}\right)-\left(A_{a} \otimes A_{a}\right) \otimes A_{a} \Rightarrow 0$. Finite gauge transformations $\left(A_{a}, B_{a}\right) \mapsto\left(\tilde{A}_{a}, \tilde{B}_{a}\right)$ are then parameterised by $M$-valued functions $p_{a}$ and $T_{p_{a}} N$-valued 1-forms $\Lambda_{p_{a}}$ and read explicitly as

$$
\begin{aligned}
\Lambda_{p_{a}}: \tilde{A}_{a} \otimes p_{a} \Rightarrow & p_{a} \otimes A_{a}-\mathrm{d} p_{a} \\
\tilde{B}_{a} \otimes \mathrm{id}_{p_{a}}= & \mu\left(\tilde{A}_{a}, \tilde{A}_{a}, p_{a}\right)+\left[\operatorname{id}_{p_{a}} \otimes B_{a}+\mu\left(p_{a}, A_{a}, A_{a}\right)\right] \circ \\
& \circ\left[-\mathrm{d} \Lambda_{p_{a}}-\Lambda_{p_{a}} \otimes \operatorname{id}_{A_{a}}-\mu\left(\tilde{A}_{a}, p_{a}, A_{a}\right)\right] \circ \\
& \circ\left[-\mathrm{id}_{\mathbf{s}\left(\mathrm{d}_{p_{a}}\right)}-\operatorname{id}_{\tilde{A}_{a}} \otimes\left(\Lambda_{p_{a}}+\operatorname{id}_{\mathrm{d} p_{a}}\right)\right] .
\end{aligned}
$$

Eventually, we combine our findings on Čech cohomology with values in a semistrict Lie 2-group with those on finite gauge transformations of local connective structures and develop full semistrict Deligne cohomology of degree 2. The corresponding Deligne cocycle and coboundary relations are concisely listed in Definitions 5.16 and 5.17.

As a first application of our results, we employ semistrict Deligne cohomology of degree 2 in a twistor description of $\mathcal{N}=(2,0)$ tensor multiplet equations in six dimensions. This is a generalisation of the previous results obtained in $[9,10]$ from strict to semistrict 
gauge 2-groups. The main result here is Theorem 6.5 in which a bijection is established between equivalence classes of certain holomorphic semistrict principal 2-bundles over a twistor space and equivalence classes of solutions to certain superconformal tensor multiplet equations in six dimensions. We hope that the latter equations may serve as an inspiration for a classical formulation of the $(2,0)$-theory.

\subsection{Outlook}

There are a number of questions arising from this paper that we plan to address in future work. First of all, there should be an integration operation, inverse to our differentiation of a Lie 2-group to a semistrict Lie 2-algebra. An obvious question is how this integration is related to that of Getzler [21] and Henriques [22]. The answer seems to be similar to that found in [25] for the strict case. Here, straightforward Lie integration of a strict Lie 2-algebra led to a Lie 2-group which is Morita equivalent to the 2-group obtained by the method of Getzler and Henriques.

As mentioned above, we hope that the detailed description of semistrict principal 2-bundles with connective structure allows for a more detailed understanding of the framework of higher gauge theory. More general theories than those derived in this present work can be considered so that the relation to alternative approaches such as the abovementioned non-Abelian tensor hierarchies should become clearer.

The most interesting dynamical theories involving connective structures on semistrict principal 2-bundles are certainly the $(2,0)$-theory and its dimensional reductions. As is common to supersymmetric theories, particular attention should be paid to the BPS subsectors of this theory. Higher analogues of instantons and monopoles, such as, for example, self-dual strings, should be studied in more detail from a mathematical perspective. Especially, the relevant topological invariants should be analysed. Some preliminary comments in this direction were already given in [26]. General considerations concerning topological invariants in higher gauge theory can be found in [27] as well as in [28] from the perspective of so-called $Q$-manifolds.

An important issue is to couple matter fields satisfyingly to higher gauge theories. Mathematically speaking, we would like to consider 2-vector bundles associated to our semistrict principal 2-bundles. Zucchini has suggested such a coupling in his approach to semistrict gauge theory [24]. However, the existence of so-called gauge rectifiers necessary in his approach could not be proved so far. Our twistor construction gives illuminating insights into how such couplings should be achieved. In particular, our approach yields the explicit example of the matter fields contained in the tensor multiplet, discusses the properties they satisfy, how gauge transformations act on them, and how they couple to connective structures.

The most important consistency test for a classical $(2,0)$-theory is to reproduce fivedimensional maximally supersymmetric Yang-Mills theory in a certain limit. This is a requirement from string theory and so far, this has neither been achieved for higher gauge theories nor for the models arising from tensor hierarchies. 


\section{Preliminaries}

In this paper, we require basics of weak 2-category theory. We shall try to be as selfcontained as possible and therefore we present the relevant definitions together with some useful examples in this section.

\subsection{Weak 2-categories}

We assume that the reader is familiar with elementary category theory. In the following, let $\mathscr{C}=\left(C_{0}, C_{1}\right)$ be a category with $C_{0}$ the objects of $\mathscr{C}$ and $C_{1}$ the morphisms of $\mathscr{C}$, respectively. In addition, the source and target maps in $\mathscr{C}$ are denoted by $\mathrm{s}$ and $\mathrm{t}$, that is, $\mathrm{s}, \mathrm{t}: C_{1} \rightarrow C_{0}$.

In higher category theory, there is always an issue concerning the level of strictness of the categorification under consideration. For example, 2-categories usually refer to strict 2-categories while weak 2-categories are often called bicategories. We shall exclusively use the terms weak 2-category, weak 2-groupoid etc. and avoid the notions of bicategory, bigroupoid etc.

We start off with the definition of a weak 2-category. The original definition stems from Benabou [29], and a good introduction to the topic can be found, for instance, in [30] and in particular in [31]. The following discussion follows mostly these references.

Definition 2.1. (Benabou [29]) $A$ weak 2-category $\mathscr{B}=\left(B_{0}, B_{1}, B_{2}\right)$ consists of a collection $B_{0}$ of objects $a, b, \ldots \in B_{0}$ and, for any pair of objects $a, b \in B_{0}$, an assignment $(a, b) \rightarrow \mathscr{C}(a, b)$ where $\mathscr{C}(a, b)=\left(C_{0}(a, b), C_{1}(a, b)\right)$ is a category. The objects $B_{0}$ are called 0-cells, the objects $C_{0}(a, b)$ are called 1-cells or 1-morphisms, and the morphisms $C_{1}(a, b)$ are called 2-cells or 2-morphisms. Composition of 2-morphisms in $C_{1}(a, b)$ will be called vertical composition and denoted by $\circ$.

In addition, $\mathscr{B}$ comes equipped with a bifunctor $\otimes: \mathscr{C}(a, b) \times \mathscr{C}(b, c) \rightarrow \mathscr{C}(a, c)$ for all $a, b, c \in B_{0}$ describing horizontal composition in $\mathscr{B}$, a functor ${ }^{1}$ id $: 1 \mapsto \operatorname{id}_{a} \in C_{0}(a, a)$ for all $a \in B_{0}$, and natural isomorphisms $\mathrm{a}$, I, and $\mathrm{r}$ defined by the following diagrams:

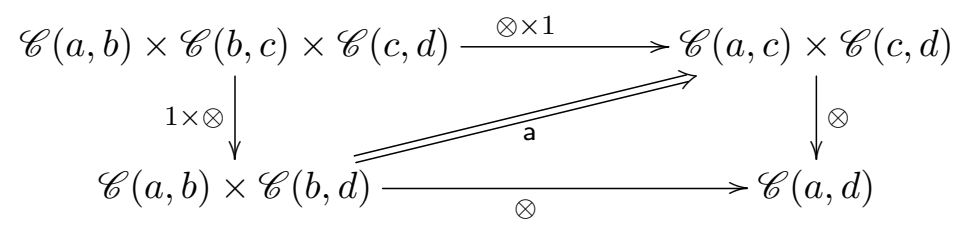

and
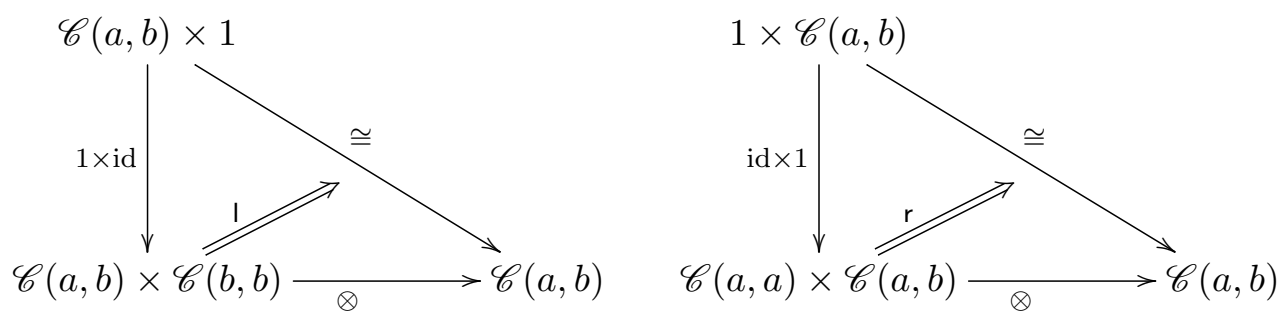

\footnotetext{
${ }^{1}$ Here, the 1 is the terminal object in the category Cat, that is, the singleton category consisting of one object $e$ and the corresponding morphism id . $_{\text {. }}$
} 
Here, the 1 attached to the arrows refers to the identity functor and $\cong$ denotes the natural isomorphisms $1 \times \mathscr{C}(a, b) \cong \mathscr{C}(a, b) \cong \mathscr{C}(a, b) \times 1$. The natural isomorphisms $\mathrm{a}$, I, and $\mathrm{r}$ are referred to as the associator, left unitor, and right unitor, and they yield the 2-cells

$$
\mathrm{a}:(x \otimes y) \otimes z \stackrel{\cong}{\Rightarrow} x \otimes(y \otimes z), \quad \mathrm{I}: x \otimes \mathrm{id}_{b} \cong x, \quad \mathrm{r}: \mathrm{id}_{a} \otimes x \stackrel{\cong}{\Rightarrow} x
$$

for $x \in C_{0}(a, b), y \in C_{0}(b, c)$, and $z \in C_{0}(c, d)$. These isomorphisms are required to satisfy the pentagon and triangle identities, that is, the diagrams

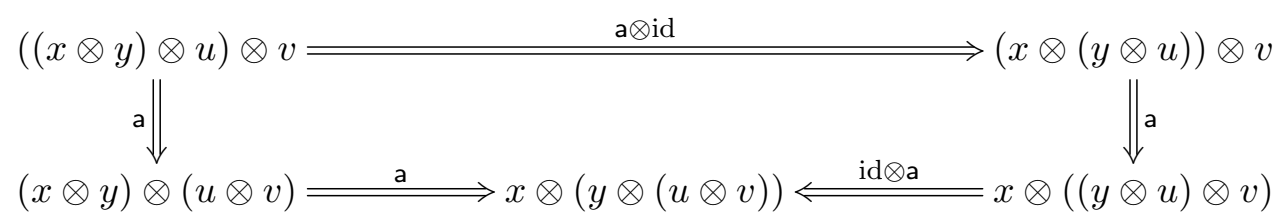

and

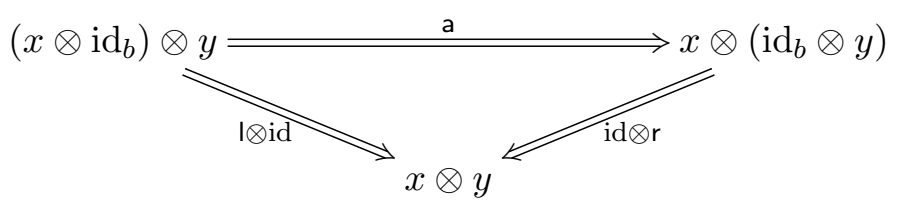

are commutative.

Remark 2.2. The fact that $\otimes$ is a bifunctor implies the so-called interchange law, that is, the diagram

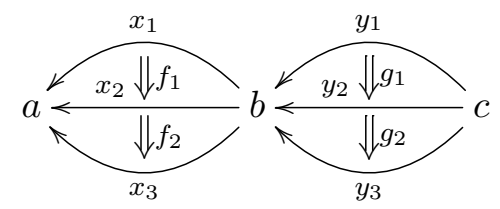

for $x_{1,2,3} \in C_{0}(a, b), y_{1,2,3} \in C_{0}(b, c)$ and $f_{1,2} \in C_{1}(a, b), g_{1,2} \in C_{1}(b, c)$ and $a, b, c \in B_{0}$ translates into

$$
\left(f_{2} \otimes g_{2}\right) \circ\left(f_{1} \otimes g_{1}\right)=\left(f_{2} \circ f_{1}\right) \otimes\left(g_{2} \circ g_{1}\right),
$$

where o denotes again vertical composition.

Remark 2.3. The naturalness of the associator a implies that diagrams of the form

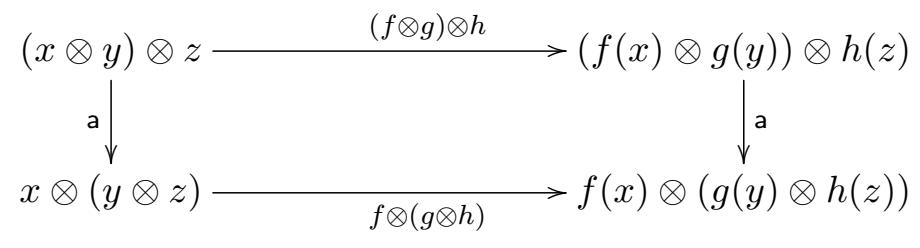

are commutative. There are similar commutative diagrams involving the unitors or a combination of the unitors and the associator.

Definition 2.4. A strict 2-category is a weak 2-category for which the associator and the left- and right-unitors are all trivial. 
Example 2.5. The standard example of a strict 2-category is Cat, regarded as a 2-category, in which the 0-cells are given by small categories, the 1-cells are functors between those, and the 2-cells are natural transformations between the latter. Horizontal composition is then the obvious composition of functors and natural transformations.

Definition 2.6. A weak 2-category with a single 0-cell can be identified with a weak monoidal category. If, in addition, the natural isomorphisms $\mathrm{a}, \mathrm{I}$, and $\mathrm{r}$ are all trivial, then we shall speak of a strict monoidal category.

The process of identifying $n$-categories with a single object or 0 -cell with $(n-1)$ categories is called looping. Below, we shall also encounter the inverse operation called delooping, see Example 2.18.

Example 2.7. An example of a strict monoidal category is the category of sets endowed with a monoidal product given either by the Cartesian product or the disjoint union of sets. Here, $B_{0}=\{e\}$ and $\mathscr{C}(e, e)$ is the category Set whose objects $C_{0}$ are sets and whose morphisms $C_{1}$ are functions between sets.

In weak 2 -categories with a single 0 -cell $e$, that is, in weak monoidal categories, we have the following result.

Proposition 2.8. (Kelly [32]) In a weak monoidal category $\mathscr{B}$, the diagrams

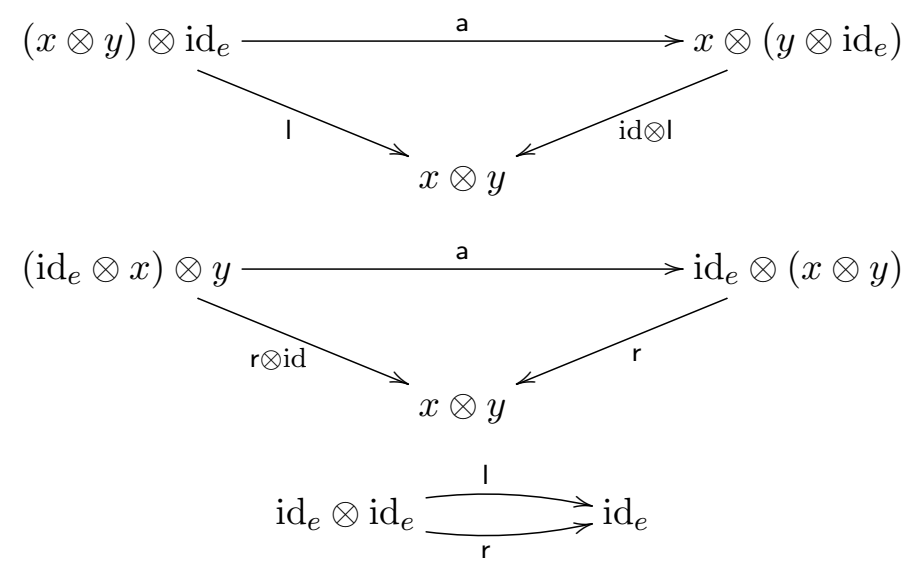

are commutative.

Morphisms between categories are called functors. Similarly, morphisms between 2categories are called 2-functors. These come in a number of variants, the most general of which are the lax 2-functors.

Definition 2.9. Let $\mathscr{B}$ and $\tilde{\mathscr{B}}$ be two weak 2-categories. $A$ lax 2 -functor $\Phi: \mathscr{B} \rightarrow \tilde{\mathscr{B}}$ is a triple $\Phi=\left(\Phi_{0}, \Phi_{1}, \Phi_{2}\right)$ consisting of a function $\Phi_{0}: B_{0} \rightarrow \tilde{B}_{0}$, a collection $\Phi_{1}$ of functors

$$
\Phi_{1}^{a b}: \mathscr{C}(a, b) \rightarrow \tilde{\mathscr{C}}\left(\Phi_{0}(a), \Phi_{0}(b)\right),
$$

and a collection $\Phi_{2}$ of 2 -cells,

$$
\begin{aligned}
\Phi_{2}^{a b c}: \Phi_{1}^{a b}(x) \tilde{\otimes} \Phi_{1}^{b c}(y) & \Rightarrow \Phi_{1}^{a c}(x \otimes y), \\
\Phi_{2}^{a}: \operatorname{id}_{\Phi_{0}(a)} & \Rightarrow \Phi_{1}^{a a}\left(\operatorname{id}_{a}\right),
\end{aligned}
$$


where $a, b, c \in B_{0}$ and $x \in C_{0}(a, b)$ and $y \in C_{0}(b, c)$ such that the following diagrams are commutative:

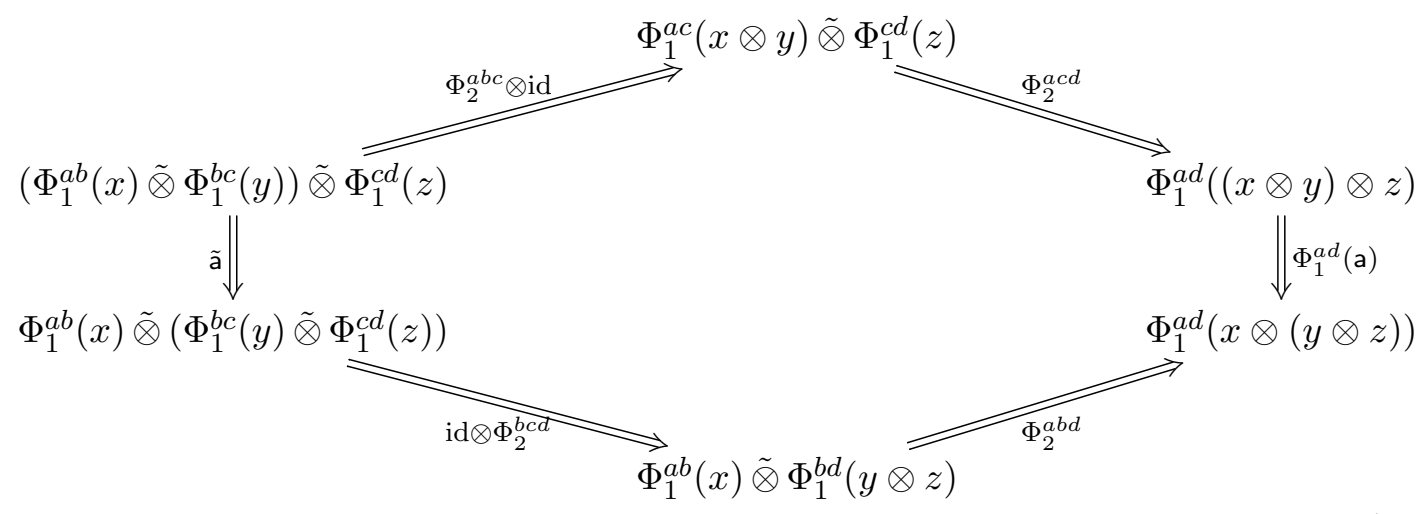

and

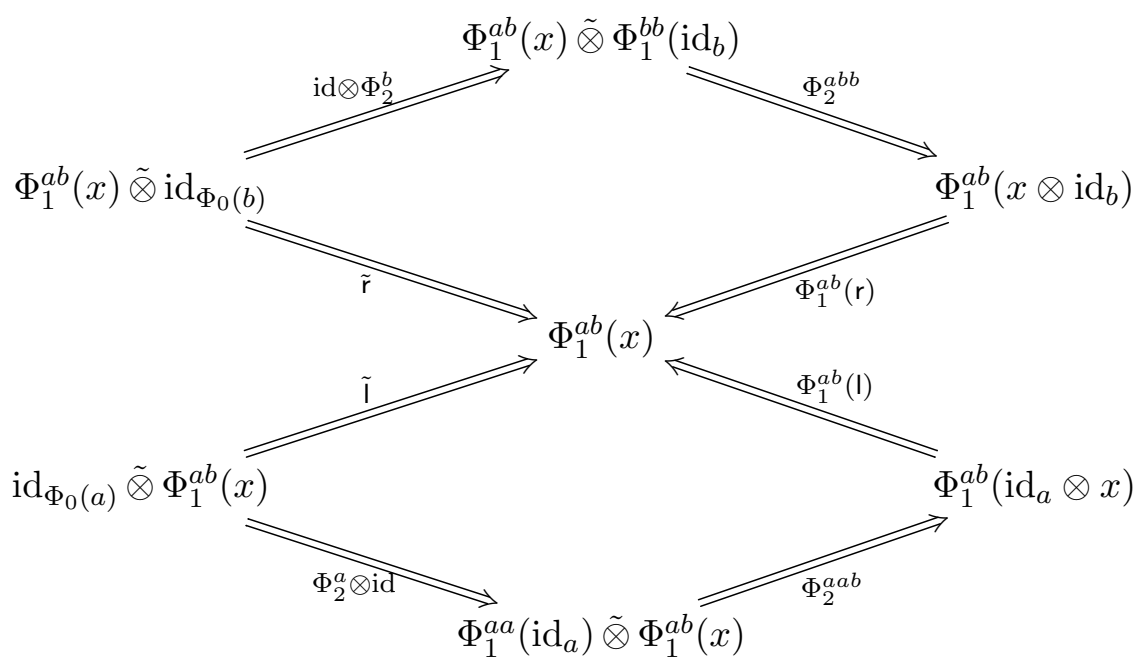

Definition 2.10. A lax 2-functor $\Phi=\left(\Phi_{0}, \Phi_{1}, \Phi_{2}\right)$ for which the 2-cells $\Phi_{2}$ are natural isomorphisms is called a weak 2-functor. ${ }^{2}$ A lax 2-functor $\Phi=\left(\Phi_{0}, \Phi_{1}, \Phi_{2}\right)$ for which the 2-cells $\Phi_{2}$ are trivial is called a strict 2-functor.

Remark 2.11. Given two lax 2-functors $\Phi=\left(\Phi_{0}, \Phi_{1}, \Phi_{2}\right): \mathscr{B} \rightarrow \tilde{\mathscr{B}}$ and $\Psi=\left(\Psi_{0}, \Psi_{1}, \Psi_{2}\right)$ : $\tilde{\mathscr{B}} \rightarrow \hat{\mathscr{B}}$, their composition $\Phi \circ \Psi$ yields another lax 2 -functor $\Xi=\left(\Xi_{0}, \Xi_{1}, \Xi_{2}\right)$ with

$$
\begin{aligned}
\Xi_{0} & =\Psi_{0} \circ \Phi_{0}: B_{0} \rightarrow \hat{B}_{0}, \\
\Xi_{1} & =\Psi_{1}^{\tilde{a} \tilde{b}} \circ \Phi_{1}^{a b}: \mathscr{C}(a, b) \rightarrow \hat{\mathscr{C}}\left(\Xi_{0}(a), \Xi_{0}(b)\right), \\
\Xi_{2}^{a b c} & =\Psi_{1}^{\tilde{a} \tilde{b}}\left(\Phi_{2}^{a b c}\right) \circ \Psi_{2}^{\tilde{a} \tilde{c} \tilde{c}}: \Xi_{1}^{a b}(x) \tilde{\otimes} \Xi_{1}^{b c}(y) \Rightarrow \Xi_{1}^{a c}(x \otimes y), \\
\Xi_{2}^{a} & =\Psi_{1}^{\tilde{a} \tilde{a}}\left(\Phi_{2}^{a}\right) \circ \Psi_{2}^{\tilde{a}}: \operatorname{id}_{\Xi_{0}(a)} \Rightarrow \Xi_{1}^{a a}\left(\operatorname{id}_{a}\right),
\end{aligned}
$$

where $a, b, c \in B_{0}$ and $\tilde{a}=\Phi_{0}(a)$ etc.

\footnotetext{
${ }^{2}$ Weak 2-functors are also known as pseudo-functors.
} 
As expected, there are also generalisations of the notion of natural transformation to the case of weak 2-categories. Because we shall need these natural 2-transformation when defining coboundary conditions, we shall introduce them now in full detail.

Definition 2.12. Let $\Phi, \Psi: \mathscr{B} \rightarrow \tilde{\mathscr{B}}$ be two lax 2-functors between two weak 2-categories $\mathscr{B}$ and $\tilde{\mathscr{B}}$. $A$ lax natural 2-transformation $\alpha: \Phi \Rightarrow \Psi$ with $\alpha=\left(\alpha_{1}, \alpha_{2}\right)$ consists of a family of 1-cells $\alpha_{1}^{a}: \Phi_{0}(a) \rightarrow \Psi_{0}(a)$ for each $a \in B_{0}$ together with a family of 2-cells $\alpha_{2}^{a b}$ defined by

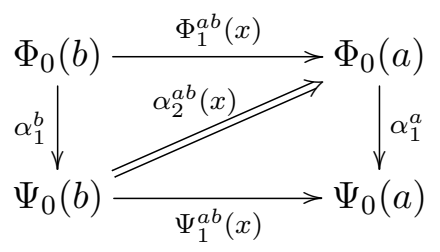

for each 1-cell $x \in C_{0}(a, b)$ in $\mathscr{B}$, such that for all $x \in C_{0}(a, b), y \in C_{0}(b, c)$ and $a, b, c \in B_{0}$ the diagrams

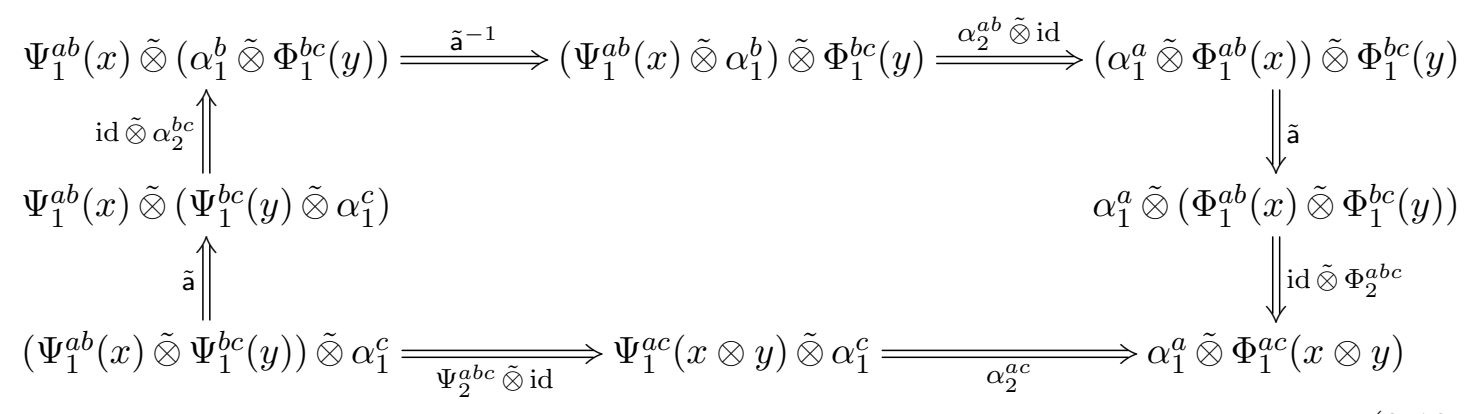

and

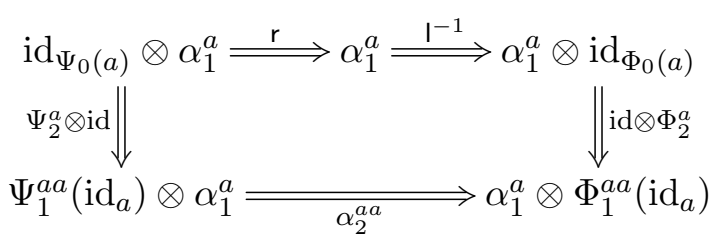

are commutative.

Definition 2.13. A lax natural 2-transformation $\alpha=\left(\alpha_{1}, \alpha_{2}\right)$ for which the 2-cells $\alpha_{2}$ are natural isomorphisms is called a weak natural 2-transformation. ${ }^{3}$ A lax natural 2transformation $\alpha=\left(\alpha_{1}, \alpha_{2}\right)$ for which the 2-cells $\alpha_{2}$ are trivial is called a strict natural 2-transformation.

The composition of natural 2-transformations is governed by the following proposition.

Proposition 2.14. Given three lax 2-functors $\Phi, \Psi, \Xi: \mathscr{B} \rightarrow \tilde{\mathscr{B}}$ between two weak 2categories $\mathscr{B}$ and $\tilde{\mathscr{B}}$ and two lax natural 2-transformations $\alpha: \Phi \Rightarrow \Psi$ and $\beta: \Psi \Rightarrow \Xi$,

\footnotetext{
${ }^{3}$ Weak natural 2-transformations are also known as pseudo-natural transformations.
} 
then there is a lax natural 2-transformation $\gamma: \Phi \Rightarrow \Xi$ such that

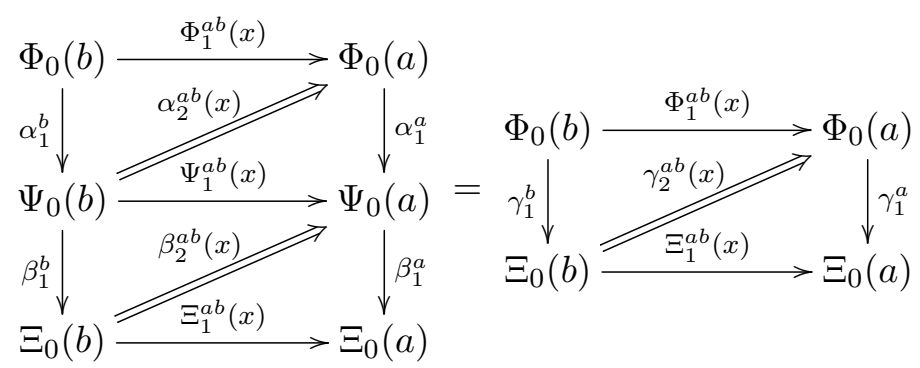

with $\gamma_{1}^{a}: \Phi_{0}(a) \rightarrow \Xi_{0}(a)$ and $\gamma_{2}^{a b}: \Xi_{1}^{a b}(x) \tilde{\otimes} \gamma_{1}^{b} \Rightarrow \gamma_{1}^{a} \tilde{\otimes} \Phi_{1}^{a b}(x)$ and

$$
\begin{aligned}
\gamma_{1}^{a} & =\beta_{1}^{a} \tilde{\otimes} \alpha_{1}^{a}, \\
\gamma_{2}^{a b} & =\tilde{\mathrm{a}}_{\beta_{1}^{a}, \alpha_{1}^{a}, \Phi^{a b}(x)}^{-1} \tilde{\mathrm{o}}\left(\operatorname{id}_{\beta_{1}^{a}} \tilde{\otimes} \alpha_{2}^{a b}(x)\right) \tilde{o} \tilde{\mathrm{a}}_{\beta_{1}^{a}, \Psi^{a b}(x), \alpha_{1}^{b}} \tilde{\circ}\left(\beta_{2}^{a b}(x) \tilde{\otimes} \operatorname{id}_{\alpha_{1}^{b}}\right) \tilde{o} \tilde{\mathrm{a}}_{\Xi^{a b}(x), \beta_{1}^{b}, \alpha_{1}^{b}}^{-1}
\end{aligned}
$$

for all $a, b \in B_{0}$ and $x \in C_{0}(a, b)$.

Proof. It is straightforward to see that $\gamma=\left(\gamma_{1}, \gamma_{2}\right)$ given in (2.13b) is a map $\gamma_{1}^{a}: \Phi_{0}(a) \rightarrow$ $\Xi_{0}(a)$ and $\gamma_{2}^{a b}: \Xi_{1}^{a b}(x) \tilde{\otimes} \gamma_{1}^{b} \Rightarrow \gamma_{1}^{a} \tilde{\otimes} \Phi_{1}^{a b}(x)$ between the lax 2-functors $\Phi$ and $\Xi$. That this is indeed a lax natural 2-transformation is a consequence of the pasting theorem for weak 2-categories, see Verity [33].

Finally, for 2-categories, it is useful to continue the sequence of 2-categories, 2-functors, 2 -transformations to 2-modifications.

Definition 2.15. Let $\Phi, \Psi: \mathscr{B} \rightarrow \tilde{\mathscr{B}}$ be two lax 2-functors between two weak 2-categories $\mathscr{B}$ and $\tilde{\mathscr{B}}$. A 2-modification between two lax natural 2-transformations $\alpha, \beta: \Phi \rightarrow \Psi$ is a collection of morphisms $\varphi_{a}: \alpha_{1}^{a} \Rightarrow \beta_{1}^{a}$ for each $a \in B_{0}$ such that

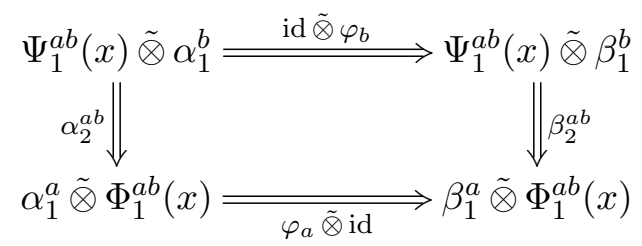

is commutative. If the morphisms $\varphi_{a}$ are invertible, we call the 2-modification invertible.

Note that composition of 2-modifications is trivially obtained by concatenation.

\section{$2.2 \quad$ Weak 2-groupoids}

In this section, we would like to introduce the notion of 2-groupoids as they play key roles in the definition of principal 2-bundles. We begin by recalling the definition of a groupoid first.

Definition 2.16. A groupoid is a small category in which every morphism is invertible.

Two important examples of groupoids that we shall frequently encounter throughout this work are those of the Čech groupoid and the delooping of a group. 
Example 2.17. The Čech groupoid relative to a covering $\mathfrak{U}:=\left\{U_{a}\right\}$ of a topological manifold $X$, denoted by $\check{\mathscr{C}}(\mathfrak{U})$ in the following, is defined to be the groupoid that has the covering sets as objects and the intersection of covering sets as morphisms. Concretely, the set of objects of $\check{\mathscr{C}}(\mathfrak{U})$ is defined to be the disjoint union $\dot{\bigcup}_{a} U_{a}:=\bigcup_{a}\left\{(x, a) \mid x \in U_{a}\right\}$ and the set of morphisms of $\check{\mathscr{C}}(\mathfrak{U})$ is defined to be the disjoint union $\dot{U}_{a, b} U_{a} \cap U_{b}:=$ $\bigcup_{a, b}\left\{(x, a, b) \mid x \in U_{a} \cap U_{b}\right\}$, together with the structure maps

$$
\begin{aligned}
\mathrm{s}(x, a, b):= & (x, b), \quad \mathrm{t}(x, a, b):=(x, a), \quad \operatorname{id}_{(x, a)}:=(x, a, a), \\
& (x, a, b) \circ(x, b, c):=(x, a, c) .
\end{aligned}
$$

Example 2.18. Let $\mathrm{G}$ be a group. The delooping of $\mathrm{G}$, denoted by $\mathrm{BG}$, is defined to be the groupoid that has only a single object, denoted by e, and the elements of the group $\mathrm{G}$ as its morphisms, $g: e \rightarrow e$ with $g \in \mathrm{G}$. In $\mathrm{BG}$, the composition of morphisms is then simply given by the group multiplication on $\mathrm{G}$, that is, $g_{2} \circ g_{1}:=g_{2} g_{1}$ for any $g_{1,2} \in \mathrm{G}$.

We are interested in the categorification of the notion of a groupoid, which is defined as follows.

Definition 2.19. A weak 2-groupoid is a weak 2-category such that all morphisms are equivalences. A weak 2-groupoid with an underlying strict 2-category is a called a strict 2-groupoid.

All morphisms being equivalences implies that the 2-cells are strictly invertible and the 1-cells are invertible up to isomorphisms. Unpacking this definition further, ${ }^{4}$ a weak 2 groupoid is a weak 2-category $\mathscr{B}$ such that for every pair of objects $a, b \in B_{0}$, the category $\mathscr{C}(a, b)$ is a groupoid. Moreover, for every pair $a, b \in B_{0}$ there is a functor ${ }^{-}: \mathscr{C}(a, b) \rightarrow$ $\mathscr{C}(b, a)$ and for every 1-cell $x \in C_{0}(a, b)$ there are natural isomorphisms $\mathrm{i}_{x}: \operatorname{id}_{a} \Rightarrow x \otimes \bar{x}$ and $\mathrm{e}_{x}: \bar{x} \otimes x \Rightarrow \mathrm{id}_{b}$ called the unit and counit. These have to satisfy coherence axioms, which state that for any 1-cell $x \in C_{0}(a, b)$ and $a, b \in B_{0}$, the diagrams

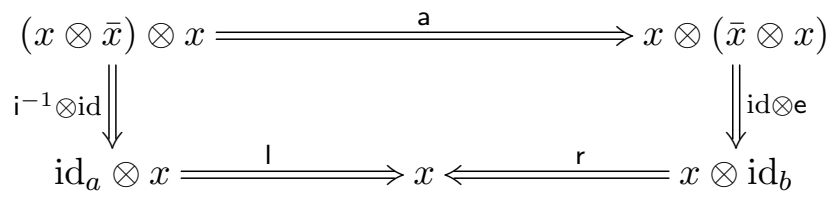

and

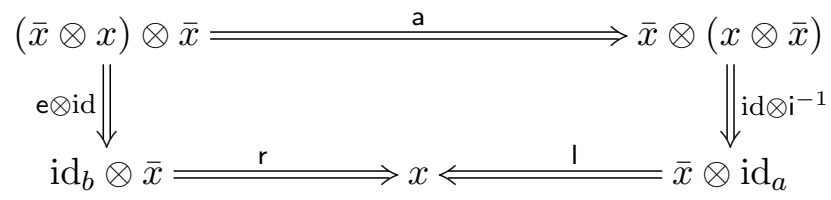

are commutative.

Example 2.20. An example of a strict 2-groupoid important in our subsequent discussion is the so-called Cech 2-groupoid. The 0- and 1-cells are given by the objects and morphisms of the Cech groupoid (see Example 2.17), and all 2-cells defined to be trivial.

\footnotetext{
${ }^{4}$ Cf. Hardie et al. [34].
} 
In Example 2.18, we have seen that any group can be viewed as a groupoid with a single object. Analogously, we give the following definition.

Definition 2.21. A weak 2-group $\mathscr{G}=(M, N)$ is the looping of a weak 2-groupoid with a single 0 -cell $\mathscr{B}=(\{e\}, M, N)$.

Remark 2.22. This definition is equivalent to that given by Baez 8 Lauda [4]. In particular, they define weak 2-groups as weak monoidal categories in which all morphisms are invertible and all objects are weakly invertible. They also introduce so-called coherent 2groups as weak monoidal categories in which all morphisms are invertible and all objects come with an adjoint equivalence. Both notions are shown to be equivalent. Our definition 2.21 uses the looping, as the weak 2-groups we are interested in will mostly appear as deloopings of coherent 2-groups in the sense of Baez \& Lauda. We shall therefore write $\mathrm{B} \mathscr{G}=(\{e\}, M, N)$ : the single 0-cell is denoted by $e$ in the following while the 1-and 2-cells are denoted by $M$ and $N$, respectively. The (monoidal) category $\mathscr{C}(e, e)$ contained in $\mathrm{B} \mathscr{G}$ is then the actual weak 2-group.

Definition 2.23. A strict 2-group is the looping of a strict 2-groupoid with a single 0-cell.

Put differently, a strict 2-group is a weak 2-group in which the unitors, the unit and counit, and the associator are all trivial. Furthermore, we will need the notion of a skeletal 2-group which is as follows.

Definition 2.24. A skeletal 2-group is a weak 2-group, in which the underlying category is skeletal.

Recall that a category is skeletal whenever all isomorphic objects are equal: for all morphisms $f$ in the category, $\mathrm{s}(f)=\mathrm{t}(f)$.

One version of Mac Lane's coherence theorem [35] states that every weak monoidal category is equivalent to a strict monoidal category. In the case of weak 2-groups, we have the following proposition from ([4], section 8.3), which can be used to classify weak Lie 2-groups.

Proposition 2.25. (Baez \& Lauda [4]) Every weak 2-group is categorically equivalent to a 'special' weak 2-group which is skeletal and in which all unitors, units, and counits are identity natural transformations. In particular, a special weak 2-group can be given in terms of a group $\mathrm{G}$, an Abelian group $\mathrm{H}$, a representation $\alpha$ of $\mathrm{G}$ as automorphisms of $\mathrm{H}$ and an element $[\mathrm{a}] \in H^{3}(\mathrm{G}, \mathrm{H})$.

In addition, we have the following result.

Proposition 2.26. (Baez \& Lauda [4]) Every weak 2-group is categorically equivalent to a strict 2-group.

The notion of 2-groups relevant for our subsequent discussion will be the following.

Definition 2.27. A semistrict 2-group is a weak 2-group in which the unitors and the unit and counit are all trivial. 
We would like to emphasise that this notion is weaker than that of a strict 2-group, because the associator remains unrestricted. For semistrict 2-groups, we have the following results.

Proposition 2.28. In the delooping of any semistrict 2-group $\mathrm{B} \mathscr{G}=(\{e\}, M, N)$, the associators $\mathrm{a}_{\mathrm{id}_{e}, m, m^{\prime}}, \mathrm{a}_{m, m^{\prime}, \mathrm{id}_{e}}, \mathrm{a}_{m, \mathrm{id}_{e}, m^{\prime}}, \mathrm{a}_{m, \bar{m}, m}$, and $\mathrm{a}_{\bar{m}, m, \bar{m}}$ are trivial for all $m, m^{\prime} \in M$.

Proof. This follows trivially by combining the pentagon and triangle diagrams together with the diagrams displayed in (2.16).

Proposition 2.29. In any semistrict 2-group $\mathscr{G}=(M, N)$ and for any 2-cell $n \in N$,

$$
n^{-1}=\mathrm{a}_{\mathrm{s}(n), \overline{\mathrm{t}(n)}, \mathrm{t}(n)} \circ\left(\left(\operatorname{id}_{\mathrm{s}(n)} \otimes \bar{n}\right) \otimes \mathrm{id}_{\mathrm{t}(n)}\right): \mathrm{t}(n) \Rightarrow \mathrm{s}(n)
$$

such that $n \circ n^{-1}=\mathrm{id}_{\mathrm{t}(n)}$ and $n^{-1} \circ n=\mathrm{id}_{\mathbf{s}(n)}$.

Proof. This follows from the proof of Proposition 20 in [4].

\subsection{Lie 2-groups}

To restrict the rather general notion of a groupoid, we can regard Lie groupoids as groupoids internal to a certain category $\mathscr{C}$. In general, a category internal to $\mathscr{C}=\left(C_{0}, C_{1}\right)$ consists of an object of objects and an object of morphisms, which are both elements in $C_{0}$. The structure maps s, t, id, and $\circ$ are given in terms of elements of $C_{1}$ and all commutative diagrams which hold in a category also hold in the internalised category. Internal functors and modifications are defined in an analogous manner. A groupoid internal to a category $\mathscr{C}$ is simply a category internal to $\mathscr{C}$, in which all the morphisms are strictly invertible.

In this manner, we can define, for instance, topological groupoids as groupoids in Top, the category of topological spaces and continuous functions between them. Similarly, Lie groupoids are defined as follows.

Definition 2.30. A Lie groupoid is a groupoid internal to Diff, the category of smooth manifolds and smooth functions between them.

Thus, Lie groupoids are groupoids in which the sets of objects and morphisms are smooth manifolds and all the structure maps are smooth.

Remark 2.31. Recall that for any category $\mathscr{K}$ there exists a strict 2-category $\mathscr{K}$ Cat with objects being categories internal to $\mathscr{K}$, morphisms being functors in $\mathscr{K}$ and 2-morphisms being natural transformations in $\mathscr{K}$. In particular, DiffCat is the strict 2-category with categories in Diff as 0-cells, functors between these as 1-cells and natural transformations between the latter as 2-cells.

We can now define weak Lie 2-groupoids and weak Lie 2-groups by internalising weak 2 -groupoids and weak 2-groups, respectively.

Definition 2.32. A weak Lie 2-groupoid is a weak 2-groupoid internal to DiffCat. A weak Lie 2-group is a weak 2-group internal to DiffCat. 
Equivalently, a weak Lie 2-group is a weak Lie 2-groupoid with a single object. Specifically, such a weak Lie 2-group consists of an object $C$ in DiffCat, a multiplication morphism $\otimes: C \times C \rightarrow C$, an identity object $\mathbb{1}$, and an inverse map ${ }^{-}: C \rightarrow C$ with respect to $\otimes$. Furthermore, we have for all objects $x, y$, and $z$ in the category $C$ the following natural isomorphisms: an associator a : $(x \otimes y) \otimes z \Rightarrow x \otimes(y \otimes z)$, left- and right-unitors $\mathrm{I}_{x}: \mathbb{1} \otimes x \rightarrow x$ and $\mathrm{r}_{x}: x \otimes \mathbb{1} \Rightarrow x$ as well as a unit and counit $\mathrm{i}_{x}: \mathbb{1} \Rightarrow x \otimes \bar{x}$ and $\mathrm{e}_{x}: \bar{x} \otimes x \rightarrow \mathbb{1}$, such that the pentagon and triangle identities as well as the first and second zig-zag identities are satisfied, cf. [4].

For our purposes, we wish to restrict the notion of a weak Lie 2-group as given in Definition 2.32 somewhat further.

Definition 2.33. A semistrict Lie 2-group is a weak 2-group internal to DiffCat such that the unitors, the unit, and the counit are all trivial.

Note that by Proposition 2.25, semistrict Lie 2-groups are still categorically equivalent to weak Lie 2-groups.

Definition 2.34. A strict Lie 2-group is a weak 2-group in DiffCat such that the associator, the unitors, the unit, and the counit are all trivial.

We recall that there is an equivalent formulation of strict Lie 2-groups in terms of crossed modules of Lie groups.

Definition 2.35. A crossed module of Lie groups is a pair of Lie groups $(\mathrm{H}, \mathrm{G})$ together with a Lie group homomorphism $5: \mathrm{H} \rightarrow \mathrm{G}$ and an action $\triangleright$ of $\mathrm{G}$ on $\mathrm{H}$ by automorphisms. The map $\partial$ is $\mathrm{G}$-equivariant and satisfies the Peiffer identity,

$$
\partial(g \triangleright h)=g \partial(h) g^{-1} \quad \text { and } \quad \partial\left(h_{1}\right) \triangleright h_{2}=h_{1} h_{2} h_{1}^{-1}
$$

for all $g \in \mathrm{G}$ and $h, h_{1}, h_{2} \in \mathrm{H}$.

Then we have the following result.

Proposition 2.36. A strict Lie 2-group is equivalent to a crossed module of Lie groups.

See Baez \& Lauda [4] for a detailed proof. We shall use an identification between strict Lie 2-groups and crossed modules of Lie groups that slightly differs from that of [4]. Given a crossed module of Lie groups $(\mathrm{H} \stackrel{\partial}{\rightarrow} \mathrm{G}, \triangleright)$, we obtain a strict Lie 2-group $\mathscr{G}=(M, N)$ by identifying $M:=\mathrm{G}$ and $N:=\mathrm{G} \ltimes \mathrm{H}$ and setting $\mathrm{s}(g, h):=\partial\left(h^{-1}\right) g, \mathrm{t}(g, h):=g$, and $\operatorname{id}_{g}=\left(g, \mathbb{1}_{\mathbf{H}}\right)$ for $h, h_{1,2} \in \mathrm{H}$ and $g, g_{1,2} \in \mathbf{G}$ together with

$$
\begin{aligned}
g_{2} \otimes g_{1} & :=g_{2} g_{1}, \\
\left(g_{2}, h_{2}\right) \otimes\left(g_{1}, h_{1}\right) & :=\left(g_{2} g_{1},\left(g_{2} \triangleright h_{1}\right) h_{2}\right), \\
\left(g, h_{2}\right) \circ\left(\partial\left(h_{2}^{-1}\right) g, h_{1}\right) & :=\left(g, h_{2} h_{1}\right) .
\end{aligned}
$$

\footnotetext{
${ }^{5}$ This homomorphism is often denoted by t. Here, however, to avoid confusion with the source and target maps of the weak 2-group, we use the symbol $\partial$.
} 
On the other hand, given a strict Lie 2 -group $\mathscr{G}=(M, N)$, we define a crossed module $(\mathrm{H} \stackrel{\partial}{\rightarrow} \mathrm{G}, \triangleright)$ by putting $\mathrm{G}:=M$ and $\mathrm{H}:=\operatorname{ker}(\mathrm{t})$ and

$$
\begin{aligned}
g_{2} g_{1}:=g_{2} \otimes g_{1}, & h_{2} h_{1}:=h_{2} \circ\left(h_{1} \otimes \mathrm{id}_{\mathbf{s}\left(h_{2}\right)}\right), \\
\partial(h):=\mathrm{s}\left(h^{-1}\right), & g \triangleright h:=\operatorname{id}_{g} \otimes h \otimes \operatorname{id}_{\bar{g}} .
\end{aligned}
$$

\section{$2.4 \quad$ Lie 2-algebras}

Apart from Lie 2-groups, we shall also be dealing with Lie 2-algebras. The most general kind of Lie 2-algebra currently in use has been defined by Roytenberg [36] as follows.

Definition 2.37. A weak Lie 2-algebra is a linear category $\mathscr{L}=\left(L_{0}, L_{1}\right)$ equipped with

(i) a bilinear functor $[\cdot, \cdot]: \mathscr{L} \times \mathscr{L} \rightarrow \mathscr{L}$ called the bracket,

(ii) a bilinear natural transformation $\mathrm{S}:[X, Y] \Rightarrow-[Y, X]$ called the alternator, and

(iii) a trilinear natural transformation $\mathrm{J}:[X,[Y, Z]] \Rightarrow[[X, Y], Z]+[Y,[X, Z]]$ called the Jacobiator

for all $X, Y, Z \in L_{0}$. These structure maps are subject to a number of coherence axioms, cf. [36].

In this paper, we are merely interested in so-called semistrict Lie 2-algebras.

Definition 2.38. A semistrict Lie 2-algebra is a weak Lie 2-algebra in which the alternator is trivial.

Instead of working directly with semistrict Lie 2-algebras and their rather involved coherence axioms, we can switch to a categorically equivalent formulation in terms of 2-term $L_{\infty}$-algebras, as was shown in [37]. The general definition of a strong homotopy Lie algebra is given in appendix A. Here, we just recall the following definition.

Definition 2.39. A 2-term $L_{\infty}$-algebra consists of a 2-term complex of vector spaces $\mathfrak{v}$ and $\mathfrak{w}$,

$$
\mathfrak{v} \stackrel{\mu_{1}}{\longrightarrow} \mathfrak{w} \stackrel{\mu_{2}}{\longrightarrow} 0
$$

where we associate gradings -1 and 0 to elements of $\mathfrak{v}$ and $\mathfrak{w}$, respectively. This complex is equipped with higher products $\mu_{1}, \mu_{2}, \mu_{3}$, which vanish except for

$$
\begin{aligned}
& \mu_{1}: \mathfrak{v} \rightarrow \mathfrak{w}, \quad \mu_{2}: \mathfrak{w} \wedge \mathfrak{w} \rightarrow \mathfrak{w}, \quad \mu_{2}: \mathfrak{v} \wedge \mathfrak{w} \rightarrow \mathfrak{v}, \\
& \mu_{3}: \mathfrak{w} \wedge \mathfrak{w} \wedge \mathfrak{w} \rightarrow \mathfrak{v} .
\end{aligned}
$$


Moreover, these products are required to satisfy the following higher homotopy Jacobi identities:

$$
\begin{aligned}
& \mu_{1}\left(\mu_{2}(w, v)\right)=\mu_{2}\left(w, \mu_{1}(v)\right), \\
& \mu_{2}\left(\mu_{1}\left(v_{1}\right), v_{2}\right)=\mu_{2}\left(v_{1}, \mu_{1}\left(v_{2}\right)\right), \\
& \mu_{1}\left(\mu_{3}\left(w_{1}, w_{2}, w_{3}\right)\right)=-\mu_{2}\left(\mu_{2}\left(w_{1}, w_{2}\right), w_{3}\right)-\mu_{2}\left(\mu_{2}\left(w_{3}, w_{1}\right), w_{2}\right)-\mu_{2}\left(\mu_{2}\left(w_{2}, w_{3}\right), w_{1}\right), \\
& \mu_{3}\left(\mu_{1}(v), w_{1}, w_{2}\right)=-\mu_{2}\left(\mu_{2}\left(w_{1}, w_{2}\right), v\right)-\mu_{2}\left(\mu_{2}\left(v, w_{1}\right), w_{2}\right)-\mu_{2}\left(\mu_{2}\left(w_{2}, v\right), w_{1}\right), \\
& \mu_{2}\left(\mu_{3}\left(w_{1}, w_{2}, w_{3}\right), w_{4}\right)-\mu_{2}\left(\mu_{3}\left(w_{4}, w_{1}, w_{2}\right), w_{3}\right)+\mu_{2}\left(\mu_{3}\left(w_{3}, w_{4}, w_{1}\right), w_{2}\right)- \\
&-\mu_{2}\left(\mu_{3}\left(w_{2}, w_{3}, w_{4}\right), w_{1}\right)= \\
&=\mu_{3}\left(\mu_{2}\left(w_{1}, w_{2}\right), w_{3}, w_{4}\right)-\mu_{3}\left(\mu_{2}\left(w_{2}, w_{3}\right), w_{4}, w_{1}\right)+\mu_{3}\left(\mu_{2}\left(w_{3}, w_{4}\right), w_{1}, w_{2}\right)- \\
&-\mu_{3}\left(\mu_{2}\left(w_{4}, w_{1}\right), w_{2}, w_{3}\right)-\mu_{3}\left(\mu_{2}\left(w_{1}, w_{3}\right), w_{2}, w_{4}\right)-\mu_{3}\left(\mu_{2}\left(w_{2}, w_{4}\right), w_{1}, w_{3}\right),
\end{aligned}
$$

where $v, v_{i} \in \mathfrak{v}$ and $w, w_{i} \in \mathfrak{w}$.

Remark 2.40. Note that for every 2-term $L_{\infty}$-algebra $\mathfrak{v} \stackrel{\mu_{1}}{\longrightarrow} \mathfrak{w}$ with products $\left(\mu_{1}, \mu_{2}, \mu_{3}\right)$, there is another 2-term $L_{\infty}$-algebra with the same underlying vector spaces $\tilde{\mathfrak{v}}:=\mathfrak{v}$ and $\tilde{\mathfrak{w}}:=\mathfrak{w}$ but with higher products $\tilde{\mu}_{1}:=-\mu_{1}, \tilde{\mu}_{2}:=\mu_{2}$, and $\tilde{\mu}_{3}:=-\mu_{3}$.

Example 2.41. A typical example of a semistrict Lie 2-algebra is the string Lie 2-algebra of a Lie algebra $\mathfrak{g}$. Here, $\mathfrak{w}=\mathfrak{g}, \mathfrak{v}=\mathbb{R}$ and the only non-trivial higher products are $\mu_{2}\left(w_{1}, w_{2}\right)=\left[w_{1}, w_{2}\right]$ and $\mu_{3}\left(w_{1}, w_{2}, w_{3}\right)=\left\langle w_{1},\left[w_{2}, w_{3}\right]\right\rangle$, where $w_{1}, w_{2}, w_{3} \in \mathfrak{w}$ and $\langle\cdot, \cdot\rangle$ is the Killing form on $\mathfrak{g}$.

Let us briefly recall the details of the equivalence between semistrict Lie 2-algebras and 2-term $L_{\infty}$-algebras. ${ }^{6}$ We start from a Lie 2-algebra $\mathscr{L}=\left(L_{0}, L_{1}\right)$ and put

$$
\mathfrak{v}:=\operatorname{ker}(\mathrm{t}) \subseteq L_{1}, \quad \mathfrak{w}:=L_{0}, \quad \text { and } \quad \mu_{1}:=-\left.\mathbf{s}\right|_{\mathfrak{v}} .
$$

The higher products are defined as follows:

$$
\begin{aligned}
\mu_{2}\left(w_{1}, w_{2}\right) & :=\left[w_{1}, w_{2}\right], \quad \mu_{2}(w, v)=-\mu_{2}(v, w):=\left[\operatorname{id}_{w}, v\right], \\
\mu_{3}\left(w_{1}, w_{2}, w_{3}\right) & :=\mathrm{J}\left(w_{1}, w_{2}, w_{3}\right)-\operatorname{id}_{\left[\left[w_{1}, w_{2}\right], w_{3}\right]+\left[w_{2},\left[w_{1}, w_{3}\right]\right]},
\end{aligned}
$$

where $w_{1}, w_{2}, w_{3}, w \in \mathfrak{w}$ and $v \in \mathfrak{v}$. This map from a semistrict Lie 2-algebra to a 2-term $L_{\infty}$-algebra can be extended to a functor $\Phi$ between the corresponding categories.

Conversely, given a 2 -term $L_{\infty}$-algebra $\mathfrak{v} \stackrel{\mu_{1}}{\longrightarrow} \mathfrak{w}$, we obtain a semistrict Lie 2-algebra $\mathscr{L}=\left(L_{0}, L_{1}\right)$ by putting

$$
\begin{aligned}
L_{0} & :=\mathfrak{w}, \quad L_{1}:=\mathfrak{v} \oplus \mathfrak{w}, \quad \mathbf{s}(w, v):=w-\mu_{1}(v), \quad \mathrm{t}(w, v):=w, \\
\operatorname{id}_{w} & :=(w, 0), \quad\left(w, v_{2}\right) \circ\left(w-\mu_{1}\left(v_{2}\right), v_{1}\right):=\left(w, v_{1}+v_{2}\right)
\end{aligned}
$$

for all $v, v_{1}, v_{2} \in \mathfrak{v}$ and $w \in \mathfrak{w}$. In addition, we set

$$
\begin{aligned}
{\left[w_{1}, w_{2}\right] } & :=\mu_{2}\left(w_{1}, w_{2}\right), \\
{\left[\left(w_{1}, v_{1}\right),\left(w_{2}, v_{2}\right)\right] } & :=\left(\mu_{2}\left(w_{1}, w_{2}\right), \mu_{2}\left(v_{1}, w_{2}\right)+\mu_{2}\left(w_{1}-\mu_{1}\left(v_{1}\right), v_{2}\right)\right), \\
\mathrm{J}\left(w_{1}, w_{2}, w_{3}\right) & :=\left(-\mu_{2}\left(\mu_{2}\left(w_{1}, w_{2}\right), w_{3}\right)-\mu_{2}\left(\mu_{2}\left(w_{3}, w_{1}\right), w_{2}\right), \mu_{3}\left(w_{1}, w_{2}, w_{3}\right)\right) .
\end{aligned}
$$

\footnotetext{
${ }^{6}$ A similar equivalence exists for weak Lie 2-algebras [36], but the resulting normalised chain complex is less convenient to work with.
} 
Again, this map from a 2-term $L_{\infty}$-algebra to a semistrict Lie 2 -algebra can be extended to a functor $\Psi$ between the corresponding categories.

We have the following results.

Proposition 2.42. (Baez \& Crans [37]) Together, the functors $\Phi$ and $\Psi$ defined above can be shown to form an equivalence, which can even be extended to an equivalence of 2-categories.

Proposition 2.43. (Baez \& Crans [37]) There is a one-to-one correspondence between equivalence classes of semistrict Lie 2-algebras and 'special' 2-term $L_{\infty}$-algebras given in terms of a Lie algebra $\mathfrak{g}$, a representation of $\mathfrak{g}$ on a vector space $\mathfrak{v}$, and an element $J$ of $H^{3}(\mathfrak{g}, \mathfrak{v})$. Here, $\mu_{1}=0, \mu_{2}$ is the Lie bracket in $\mathfrak{g}$ or the action on $\mathfrak{v}$, and $\mu_{3}=J$.

Semistrict Lie 2-algebras can be restricted further to obtain strict Lie 2-algebras.

Definition 2.44. A strict Lie 2-algebra is a weak Lie 2-algebra with trivial alternator and trivial Jacobiator.

Our above discussion immediately implies that strict Lie 2-algebras are equivalent to 2 -term $L_{\infty}$-algebras with trivial product $\mu_{3}$, which in turn, can be encoded in a differential crossed module.

Definition 2.45. The differential crossed module of a crossed module of Lie groups is obtained by applying the tangent functor to the crossed module.

In particular, given a crossed module of Lie groups $(H \stackrel{\partial}{\rightarrow} G, \triangleright)$, the tangent functor yields a differential crossed module ${ }^{7}(\mathfrak{h} \stackrel{\partial}{\rightarrow} \mathfrak{g}, \triangleright)$, where $\mathfrak{h}:=\operatorname{Lie}(H)$ and $\mathfrak{g}:=\operatorname{Lie}(\mathrm{G})$. The maps $\partial$ and $\triangleright$ satisfy

$$
\partial(X \triangleright Y)=[X, \partial(Y)] \text { and } \partial\left(Y_{1}\right) \triangleright Y_{2}=\left[Y_{1}, Y_{2}\right],
$$

where $X \in \mathfrak{g}$ and $Y, Y_{1,2} \in \mathfrak{h}$.

The differential crossed module corresponding to a 2-term $L_{\infty}$-algebra $\mathfrak{v} \stackrel{\mu_{1}}{\longrightarrow} \mathfrak{w}$ with trivial $\mu_{3}$ is obtained by identifying $\mathfrak{h}, \mathfrak{g}$, and $\partial$ with $\mathfrak{v}, \mathfrak{w}$, and $\mu_{1}$ as well as

$$
\left[w_{1}, w_{2}\right]:=\mu_{2}\left(w_{1}, w_{2}\right), \quad v \triangleright w:=\mu_{2}(v, w) \quad \text { and } \quad\left[v_{1}, v_{2}\right]:=\mu_{2}\left(\mu_{1}\left(v_{1}\right), v_{2}\right)
$$

for $v_{1}, v_{2}, v \in \mathfrak{v}=\mathfrak{h}$ and $w_{1}, w_{2}, w \in \mathfrak{w}=\mathfrak{g}$. This identification is readily inverted.

\section{Principal 2-bundles with Lie 2-groups}

We come now to the discussion of principal 2-bundles with weak structure 2-groups over smooth manifolds. An earlier description of general 2-bundles from a slightly different point of view can be found in Bartels [3]. In the following, let $X$ be a smooth manifold and let $\mathfrak{U}=\left\{U_{a}\right\}$ be a covering of $X$.

\footnotetext{
${ }^{7}$ Our notation does not distinguish between the maps $\partial, \triangleright$ and their differentials.
} 


\subsection{Principal bundles as functors}

Recall that a Cech $p$-cochain with values in a group $\mathrm{G}$ on $X$ relative to the covering $\mathfrak{U}$ is a set of smooth G-valued functions on all non-empty intersections $U_{a_{0}} \cap \cdots \cap U_{a_{p}} .{ }^{8}$ We then give the following definition.

Definition 3.1. A Čech 1-cocycle is a Čech 1-cochain $\left\{g_{a b}\right\}$ consisting of smooth maps $g_{a b}: U_{a} \cap U_{b} \rightarrow \mathrm{G}$ such that

$$
g_{a b} g_{b c}=g_{a c} \text { on } U_{a} \cap U_{b} \cap U_{c} .
$$

Two Čech 1-cocycles $\left\{g_{a b}\right\}$ and $\left\{\tilde{g}_{a b}\right\}$ are cohomologous or equivalent if and only if there is a Čech 0-cochain $\left\{g_{a}\right\}$ consisting of smooth maps $g_{a}: U_{a} \rightarrow \mathrm{G}$ such that

$$
g_{a b}=g_{a} \tilde{g}_{a b} g_{b}^{-1} .
$$

The first Čech cohomology set, denoted by $H^{1}(\mathfrak{U}, \mathrm{G})$, is defined as the set of Čech 1-cocycles modulo this equivalence.

Cech cohomology sets can be rendered independent of the covering by taking the direct limit over all coverings $\mathfrak{U}$ of $X$. We then write $H^{1}(X, \mathrm{G})$ instead of $H^{1}(\mathfrak{U}, \mathrm{G})$, that is,

$$
H^{1}(X, \mathrm{G})=\underset{\mathfrak{U}}{\lim } H^{1}(\mathfrak{U}, \mathrm{G})
$$

Elements of $H^{1}(X, \mathrm{G})$ are also known as (sets of) transition functions of principal bundles with structure group $\mathrm{G}$ (or principal G-bundles for short), and it is well-known that principal G-bundles over $X$ can be identified with an elements in $H^{1}(X, \mathrm{G})$. To allow for a categorification of this picture, we switch to a functorial description of principal bundles.

Definition 3.2. A smooth principal bundle $\Phi$ with structure group $\mathrm{G}$ is a smooth functor $\Phi$ from the Cech groupoid to the Lie groupoid BG. ${ }^{9}$ Any two principal bundles are called equivalent if and only if there is a natural isomorphism between their defining functors.

Definition 3.2 is well-known from the description of principal bundles in terms of classifying spaces [38]. Explicitly, we have a functor

$$
\Phi: \check{\mathscr{C}}(\mathfrak{U}) \rightarrow \text { BG }
$$

and we set $e_{a}:=\Phi(x, a)$ and $g_{a b}:=\Phi(x, a, b)$. Because $\Phi$ is a functor, we immediately arrive at the cocycle conditions (3.1) as well as $\Phi(x, a, a)=\mathrm{id}_{\Phi(x, a)}=\mathbb{1}_{\mathrm{G}} \in \mathrm{G}$. In addition, two functors $\Phi$ and $\Psi$ corresponding to principal bundles are equivalent if and only if

\footnotetext{
${ }^{8}$ If not stated otherwise, we shall always assume that intersections of patches are non-empty from now on.

${ }^{9}$ See Examples 2.17 and 2.18 for the relevant definitions.
} 
there is a natural isomorphism $\alpha: \tilde{\Phi} \rightarrow \Phi$. Defining $e_{a}:=\Phi(x, a), g_{a b}=\Phi(x, a, b)$, and $g_{a}:=\alpha_{(x, a)}: \tilde{\Phi}(x, a) \rightarrow \Phi(x, a)$, the following diagram is commutative:

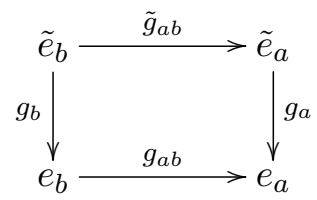

In formulæ, this is

$$
g_{a} \tilde{g}_{a b}=g_{a b} g_{b},
$$

which amounts to (3.2). We thus arrive at the following statement, which motivates our Definition 3.2.

Proposition 3.3. Denoting the set of equivalence classes of smooth functors between $\check{\mathscr{C}}(\mathfrak{U})$ and $\mathrm{BG}$ by $[\check{\mathscr{C}}(\mathfrak{U}) \rightarrow \mathrm{BG}]$, we have

$$
H^{1}(\mathfrak{U}, \mathrm{G}) \cong[\check{\mathscr{C}}(\mathfrak{U}) \rightarrow \mathrm{BG}]
$$

Other conventional definitions are now also straightforwardly rephrased.

Definition 3.4. A principal bundle is called trivial if and only if its defining functor is equivalent to the functor

$$
\Phi(x, a)=e_{a} \quad \text { and } \quad \Phi(x, a, b)=\mathbb{1}_{\mathrm{G}} .
$$

Concretely, a principal bundle is trivial whenever there is a natural isomorphism $\alpha=\left\{g_{a}\right\}$ such that

$$
g_{a}=g_{a b} g_{b}
$$

Finally, let $\phi: X \rightarrow Y$ be a smooth map between two smooth manifolds $X$ and $Y$. Let $\mathfrak{U}_{Y}$ be a covering of $Y$. Then we can construct a covering $\mathfrak{U}_{X}$ of $X$ from the pre-images of the patches in $\mathfrak{U}_{Y}$ under $\phi$. This yields a morphism of groupoids $\check{\mathscr{C}}\left(\mathfrak{U}_{X}\right) \rightarrow \check{\mathscr{C}}\left(\mathfrak{U}_{Y}\right)$.

Definition 3.5. The pullback of a principal bundle $\Phi$ over $Y$ with respect to an open covering $\mathfrak{U}_{Y}$ along a smooth map $\phi: X \rightarrow Y$ is the composition $\Phi \circ \phi_{\mathfrak{U}}$, where $\phi_{\mathfrak{U}}$ is the groupoid morphism induced by $\phi$.

Definition 3.6. The restriction of a principal bundle $\Phi$ over a manifold $X$ to a submanifold $Y$ of $X$ is the pullback of $\Phi$ along the embedding map $Y \hookrightarrow X$.

\subsection{Principal 2-bundles as 2-functors}

The reformulation of principal bundles with structure group $\mathrm{G}$ in terms of functors between the Cech groupoid and the Lie groupoid BG is a good starting point for categorifying the notion of principal bundles. We can simply regard the Čech groupoid as an $n$-groupoid and take an $n$-functor to a Lie $n$-groupoid with a single 0 -cell. In the following, we shall develop the case $n=2$ in detail. Note that our discussion will first centre around weak principal 2-bundles which we shall define in terms of weak 2 -functors. In the following, we 
shall consider the delooping $\mathrm{B} \mathscr{G}=(\{e\}, M, N)$ of a weak Lie 2-group $\mathscr{G}=(M, N)$, which is a weak Lie 2-groupoid with a single object $e$. As in section 2, we shall denote horizontal and vertical composition in $\mathrm{B} \mathscr{G}$ by $\otimes$ and $\circ$, respectively.

Principal 2-bundles will be described by Čech cocycles with values in $\mathscr{G}$. We therefore start by giving the following definition.

Definition 3.7. A degree-p Čech cochain with values in a weak Lie 2-group $\mathscr{G}=(M, N)$ consists of a smooth $M$-valued degree- $(p-1)$ Cech cochain $\left\{m_{a_{0} \cdots a_{p-1}}\right\}$, a smooth $N$ -

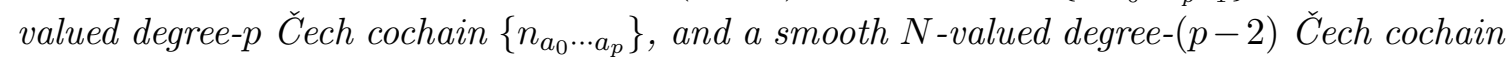
$\left\{n_{a_{0} \cdots a_{p-2}}\right\}$.

In the following, we are interested in the case $p=2$, for which we have a triple

$$
\left(\left\{m_{a b}\right\},\left\{n_{a b c}\right\},\left\{n_{a}\right\}\right) .
$$

These cochains generalise the usual Čech cochains appearing in the definition of an ordinary principal bundle in the way that is familiar from strict principal 2-bundles: the $\left\{m_{a b}\right\}$ are generalised transition functions on overlaps, the $\left\{n_{a b c}\right\}$ are the gluing isomorphisms on triple overlaps and the $\left\{n_{a}\right\}$ are the isomorphisms between the unit in $M$ and the transition functions $\left\{m_{a a}\right\}$.

To derive the explicit cocycle and coboundary conditions appropriate for weak Lie 2 -groups, we again employ the functorial approach.

Definition 3.8. A smooth weak principal 2-bundle $\Phi$ with structure 2-group $\mathscr{G}$ relative to the covering $\mathfrak{U}$ is a smooth weak 2-functor $\Phi$ from the $\breve{C}$ ech 2-groupoid $\check{\mathscr{C}}(\mathfrak{U})$ to BG .

Let us be more specific. We have a weak 2 -functor ${ }^{10}$

$$
\Phi: \check{\mathscr{C}}(\mathfrak{U}) \rightarrow \mathrm{B} \mathscr{G}
$$

consisting of a function $\Phi_{0}(x, a)$, functors $\Phi_{1}(x, a, b)$ and 2-cells $\Phi_{2}$. Note that the 0-cells of $\mathrm{B} \mathscr{G}=(\{e\}, M, N)$ and the 2-cells of $\check{\mathscr{C}}(\mathfrak{U})$ are trivial and we shall denote them by $e$. We can therefore specify $\Phi$ in terms of constant functions $e_{a}:=\Phi_{0}(x, a): U_{a} \rightarrow e$, functions $m_{a b}:=\left.\Phi_{1}(x, a, b)\right|_{M}: U_{a} \cap U_{b} \rightarrow M$, and constant functions $e_{a b}:=\left.\Phi_{1}(x, a, b)\right|_{N}: e \rightarrow \mathrm{id}_{\mathrm{id}_{e}}$ together with invertible functions $n_{a b c}: U_{a} \cap U_{b} \cap U_{c} \rightarrow N$ and $n_{a}: U_{a} \rightarrow N$ describing the 2 -cell $\Phi_{2}$. Because $\operatorname{id}_{(x, a)}=(x, a, a)$, we have by definition $\Phi_{1}\left(\operatorname{id}_{(x, a)}\right)=\Phi_{1}(x, a, a)=m_{a a}$. The fact that $\Phi_{1}$ is a functor implies $\mathrm{id}_{m_{a b}}=\operatorname{id}_{\Phi_{1}(x, a, b)}=\Phi_{1}\left(\operatorname{id}_{(x, a, b)}\right)$. Finally, with $\Phi_{1}((x, a, b) \circ(x, b, c))=\Phi_{1}(x, a, c)=m_{a c}$, we have the natural isomorphisms

$$
\begin{aligned}
n_{a b c}: m_{a b} \otimes m_{b c} & \Rightarrow m_{a c}, \\
n_{a}: \operatorname{id}_{e_{a}} & \Rightarrow m_{a a},
\end{aligned}
$$

with $\operatorname{id}_{e_{a}} \in M$.

\footnotetext{
${ }^{10}$ Cf. Definition 2.10 .
} 
The following diagrams, which arise from (2.9) with a, r, and I being trivial in $\check{\mathscr{C}}(\mathfrak{U})$, are commutative:

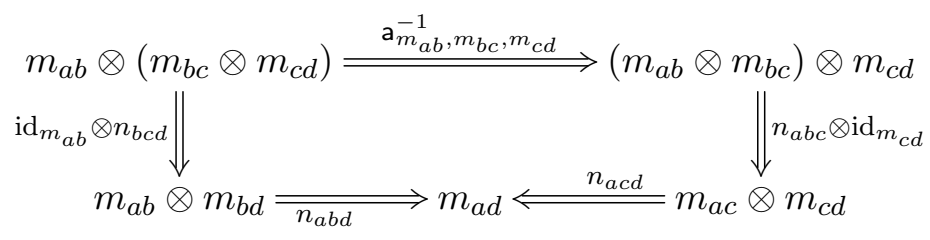

and

$$
m_{a b} \otimes \mathrm{id}_{e_{b}} \underset{\mathrm{I}_{m_{a b}}}{\stackrel{\mathrm{id}_{m_{a b} \otimes n_{b}}}{\Longrightarrow}} m_{a b} \otimes m_{b b} \quad \|_{m_{a b}} n_{a b b} \text { and } \quad \operatorname{id}_{e_{a}} \otimes m_{a b} \underbrace{\stackrel{n_{a} \otimes \mathrm{id}_{m_{a b}}}{\longrightarrow}}_{\mathrm{r}_{m_{a b}}} m_{a a} \otimes m_{a b}
$$

In formulæ, this reads as

$$
n_{a c d} \circ\left(n_{a b c} \otimes \mathrm{id}_{m_{c d}}\right) \circ \mathrm{a}_{m_{a b}, m_{b c}, m_{c d}}^{-1}=n_{a b d} \circ\left(\mathrm{id}_{m_{a b}} \otimes n_{b c d}\right)
$$

and

$$
n_{a b b} \circ\left(\operatorname{id}_{m_{a b}} \otimes n_{b}\right)=I_{m_{a b}} \quad \text { and } \quad n_{a a b} \circ\left(n_{a} \otimes \mathrm{id}_{m_{a b}}\right)=\mathrm{r}_{m_{a b}} .
$$

Definition 3.9. A $\mathscr{G}$-valued degree-2 Čech cochain $\left(\left\{m_{a b}\right\},\left\{n_{a b c}\right\},\left\{n_{a}\right\}\right)$ that satisfies the equations (3.12) and (3.14) is called a $\mathscr{G}$-valued degree-2 Čech cocycle. The equations (3.12) and (3.14) are called the cocycle conditions of a weak principal 2-bundle $\Phi$ defined by $\left(\left\{m_{a b}\right\},\left\{n_{a b c}\right\},\left\{n_{a}\right\}\right)$ and the degree-2 Cech cocycle $\left(\left\{m_{a b}\right\},\left\{n_{a b c}\right\},\left\{n_{a}\right\}\right)$ is called its transition functions.

Pushing the analogy with the case of principle bundles further, we derive equivalence relations between weak principal 2-bundles from natural 2-transformations.

Definition 3.10. Any two weak principal 2-bundles are called equivalent if and only if there is a smooth weak natural 2-transformation between their defining weak 2-functors.

Explicitly, for weak principal 2-bundles $\Phi$ and $\tilde{\Phi}$, such a natural 2-transformation $\alpha: \tilde{\Phi} \rightarrow \Phi$ is given by the following data: we have 1-cells $\left\{m_{a}\right\}$ and 2-cells $\left\{n_{a b}\right\}$,

$$
\begin{aligned}
m_{a}: \tilde{e}_{a} & \rightarrow e_{a}, \\
n_{a b}: m_{a b} \otimes m_{b} & \Rightarrow m_{a} \otimes \tilde{m}_{a b},
\end{aligned}
$$

defined by the diagram

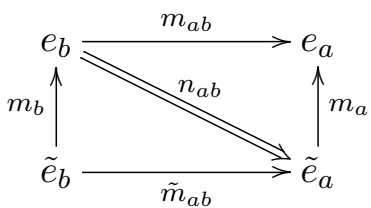


The coherence conditions for natural 2-transformations require also the diagrams

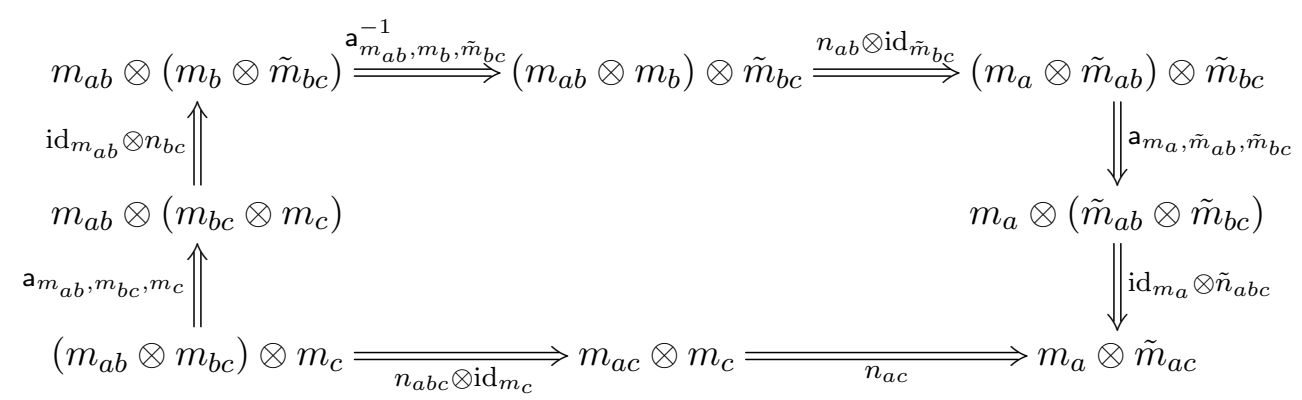

and

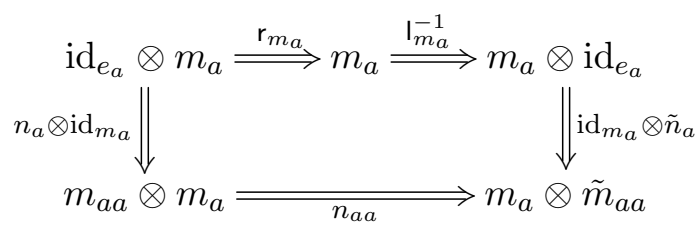

to be commutative. In formulæ, this amounts to

$$
\begin{aligned}
n_{a c} \circ\left(n_{a b c} \otimes \mathrm{id}_{m_{c}}\right)= & \left(\operatorname{id}_{m_{a}} \otimes \tilde{n}_{a b c}\right) \circ \mathrm{a}_{m_{a}, \tilde{m}_{a b}, \tilde{m}_{b c}} \circ\left(n_{a b} \otimes \mathrm{id}_{\tilde{m}_{b c}}\right) \circ \\
& \circ \mathrm{a}_{m_{a b}, m_{b}, \tilde{m}_{b c}}^{-1} \circ\left(\mathrm{id}_{m_{a b}} \otimes n_{b c}\right) \circ \mathrm{a}_{m_{a b}, m_{b c}, m_{c}}
\end{aligned}
$$

and

$$
n_{a a} \circ\left(n_{a} \otimes \mathrm{id}_{m_{a}}\right)=\left(\mathrm{id}_{m_{a}} \otimes \tilde{n}_{a}\right) \circ \mathrm{I}_{m_{a}}^{-1} \circ \mathrm{r}_{m_{a}} .
$$

Definition 3.11. Any two $\mathscr{G}$-valued degree-2 Čech cocycles $\left(\left\{m_{a b}\right\},\left\{n_{a b c}\right\},\left\{n_{a}\right\}\right)$ and $\left(\left\{\tilde{m}_{a b}\right\},\left\{\tilde{n}_{a b c}\right\},\left\{\tilde{n}_{a}\right\}\right)$ are called equivalent or cohomologous if and only if there is a $\mathscr{G}_{-}$ valued degree-1 Cech cochain $\left(\left\{m_{a}\right\},\left\{n_{a b}\right\}\right)$ such that the equations (3.15) and (3.18) are satisfied. These equations are called the coboundary conditions for a weak principal 2bundle $\Phi$ defined by $\left(\left\{m_{a b}\right\},\left\{n_{a b c}\right\},\left\{n_{a}\right\}\right)$, and, slightly deviating from the usual nomenclature, the degree-1 $\check{C}$ ech cochain $\left(\left\{m_{a}\right\},\left\{n_{a b}\right\}\right)$ is called a degree-2 Čech coboundary.

Definition 3.12. A weak principal 2-bundle that is equivalent to the weak principal 2bundle specified by the functor

$$
\left\{m_{a b}=\operatorname{id}_{e_{a}}\right\}, \quad\left\{n_{a b c}=\mathrm{I}_{\mathrm{id}_{e_{a}}}=\mathrm{rid}_{e_{a}}\right\}, \quad \text { and } \quad\left\{n_{a}=\operatorname{id}_{\mathrm{id}_{e_{a}}}\right\}
$$

is called trivial.

We shall give explicit formulæ for the transition functions of trivial bundles in the case of semistrict principal 2-bundles later on.

Note that for strict 2-bundles, the 2-cells $\left\{n_{a}\right\}$ can always be chosen to be trivial, as was done, for instance, in $[9,10]$. The same is true here, as we verify now.

Lemma 3.13. Consider transition functions $\left(\left\{m_{a b}\right\},\left\{n_{a b c}\right\},\left\{n_{a}\right\}\right)$ of a weak principal 2bundle $\Phi$. The triple $\left(\left\{\tilde{m}_{a b}\right\},\left\{\tilde{n}_{a b c}\right\},\left\{\tilde{n}_{a}\right\}\right)$ which agrees with that of $\Phi$ except for

$$
\left\{\tilde{m}_{a a}=\mathrm{id}_{e_{a}}\right\}, \quad\left\{\tilde{n}_{a a b}=\mathrm{r}_{\tilde{m}_{a b}}\right\}, \quad\left\{\tilde{n}_{a b b}=\mathrm{I}_{\tilde{m}_{a b}}\right\}, \quad \text { and } \quad\left\{\tilde{n}_{a}=\operatorname{id}_{\mathrm{id}_{e_{a}}}\right\},
$$


defines another weak principal 2-bundle $\tilde{\Phi}$. In addition, these equations imply

$$
\left\{\tilde{n}_{a a a}=\mathrm{r}_{\mathrm{id}_{e_{a}}}=\mathrm{I}_{\mathrm{id}_{e_{a}}}\right\}
$$

Proof. One readily checks that the cocycle conditions (3.12) and (3.14) are satisfied for any possible doubling of indices.

Definition 3.14. For every weak principal 2-bundle $\Phi$, the weak principal 2-bundle $\tilde{\Phi}$ obtained from the construction of Lemma 3.13 is called the normalisation of $\Phi$.

Proposition 3.15. Every weak principal 2-bundle is equivalent to its normalisation.

Proof. A natural 2-transformation that yields the equivalence is given by

$$
\left\{m_{a}=\operatorname{id}_{e_{a}}\right\} \quad \text { and } \quad\left\{n_{a b}=\left\{\begin{array}{lll}
\mathrm{r}_{\tilde{m}_{a b}}^{-1} \circ \mathrm{I}_{m_{a b}} & \text { for } & a \neq b \\
n_{a}^{-1} \otimes \mathrm{id}_{\mathrm{id}_{e_{a}}} & \text { for } & a=b
\end{array}\right\} .\right.
$$

Note that $\tilde{m}_{a b}=m_{a b}$ for $a \neq b$. As one may check, the coboundary conditions (3.15) and (3.18) are indeed satisfied. Note that the choice of $n_{a b}$ in this transformation for $a \neq b$ is not unique.

Corollary 3.16. Every weak principal 2-bundle is locally trivialisable.

Proof. By Proposition 3.15, a weak principal 2-bundle $\Phi$ is equivalent to its normalisation, for which we have

$$
n_{a a a}=\mathrm{l}_{\mathrm{id}_{e_{a}}}=\mathrm{rid}_{e_{a}}, \quad n_{a}=\mathrm{id}_{\mathrm{id}_{e_{a}}}, \quad \text { and } \quad m_{a a}=\mathrm{id}_{e_{a}} .
$$

on any $U_{a} \in \mathfrak{U}$. Thus, the weak principal 2-bundle is locally equivalent to a trivial one.

Recall that trivial principal bundles with structure group $\mathrm{G}$ are described by transition functions $\left\{g_{a b}\right\}$ of the form $g_{a b}=g_{a} g_{b}^{-1}$, where $\left\{g_{a}\right\}$ is a G-valued Čech 0-cochain. Note that the $g_{a}$ can be multiplied by a (global) G-valued function from the right, leaving $g_{a b}=g_{a} g_{b}^{-1}$ invariant. This is an equivalence relation, which is described by modifications in functorial language.

The corresponding equivalence relations are more comprehensive in the case of principal 2-bundles, as we shall see in the following. Consider two equivalent weak principal 2-bundles $\Phi$ and $\tilde{\Phi}$ with natural 2-transformations $\alpha: \tilde{\Phi} \rightarrow \Phi$ and $\tilde{\alpha}: \tilde{\Phi} \rightarrow \Phi$ between them. A weak 2-modification $\varphi: \alpha \Rightarrow \tilde{\alpha}$ is given by a smooth map $\varphi: \alpha \rightarrow \tilde{\alpha}$ that assigns to every object $(x, a) \in \check{\mathscr{C}}(\mathfrak{U})$ a 2 -morphism $\varphi_{(x, a)}: \alpha_{(x, a)} \Rightarrow \tilde{\alpha}_{(x, a)}$. We set $o_{a}:=\varphi_{(x, a)}$ so that $o_{a}: m_{a} \Rightarrow \tilde{m}_{a}$. Moreover, the following diagram is required to be commutative:

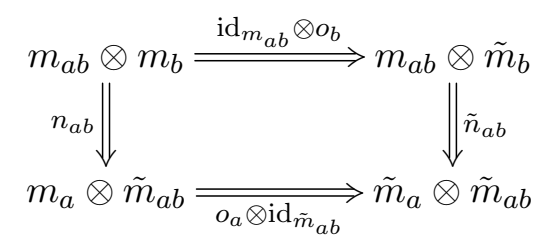

that is,

$$
\tilde{n}_{a b} \circ\left(\mathrm{id}_{m_{a b}} \otimes o_{b}\right)=\left(o_{a} \otimes \mathrm{id}_{\tilde{m}_{a b}}\right) \circ n_{a b} .
$$


Definition 3.17. Any two $\mathscr{G}$-valued degree-2 Čech coboundaries $\left(\left\{m_{a}\right\},\left\{n_{a b}\right\}\right)$ and $\left(\left\{\tilde{m}_{a}\right\}\right.$, $\left.\left\{\tilde{n}_{a b}\right\}\right)$ between any two $\mathscr{G}$-valued degree-2 Cech cocycles $\left(\left\{m_{a b}\right\},\left\{n_{a b c}\right\},\left\{n_{a}\right\}\right)$ and $\left(\left\{\tilde{m}_{a b}\right\}\right.$, $\left.\left\{\tilde{n}_{a b c}\right\},\left\{\tilde{n}_{a}\right\}\right)$ are said to be equivalent if and only if there is a $\mathscr{G}$-valued degree-0 Cech cochain $\left\{o_{a}\right\}$ such that equations (3.25) are satisfied. Such a degree-0 Čech cochain $\left\{o_{a}\right\}$ is called a degree-2 Cech modification.

To define pullbacks and restrictions of weak principal 2-bundles, we proceed just as in the case of the functorial description of principal bundles; see Definitions 3.5 and 3.6. Recall that given a smooth map $\phi: X \rightarrow Y$ and a covering $\mathfrak{U}_{Y}$ of $Y$, the pre-images of the patches in $\mathfrak{U}_{Y}$ form a covering of $\mathfrak{U}_{X}$. The resulting groupoid morphisms $\check{\mathscr{C}}\left(\mathfrak{U}_{X}\right) \rightarrow \check{\mathscr{C}}\left(\mathfrak{U}_{Y}\right)$ can be extended to a strict 2 -functor $\phi_{\mathfrak{U}}$. Therefore, we give the following definitions.

Definition 3.18. The pullback of a weak principal 2-bundle $\Phi$ over $Y$ with respect to an open covering $\mathfrak{U}_{Y}$ along a map $\phi: X \rightarrow Y$ is the composition of 2-functors $\Phi \circ \phi_{\mathfrak{U}}$.

Definition 3.19. The restriction of a weak principal 2-bundle $\Phi$ over a manifold $X$ to a submanifold $Y$ inside $X$ is the pullback of $\Phi$ along the embedding map $Y \hookrightarrow X$.

\subsection{Semistrict and strict principal 2-bundles}

We shall be specifically interested in weak principal 2-bundles with semistrict structure 2 -groups. This implies a number of simplifications, which we shall discuss in the following.

Definition 3.20. A semistrict principal 2-bundle is a normalised weak principal 2-bundle with semistrict structure 2-group $\mathscr{G}$.

Explicitly, we have a weak 2-functor $\Phi$ described by a Čech 2-cocycle (transition functions) $\left(\left\{m_{a b}\right\},\left\{n_{a b c}\right\},\left\{n_{a}\right\}\right)$ with values in $\mathscr{G}$ such that

$$
\left\{m_{a a}=\mathrm{id}_{e_{a}}\right\}, \quad\left\{n_{a a b}=\mathrm{id}_{m_{a b}}\right\}, \quad\left\{n_{a b b}=\mathrm{id}_{m_{a b}}\right\}, \quad \text { and } \quad\left\{n_{a}=\mathrm{id}_{\mathrm{id}_{e_{a}}}\right\} .
$$

The cocycle conditions for this type of principal 2-bundle then read as

$$
\begin{aligned}
n_{a b c}: m_{a b} \otimes m_{b c} & \Rightarrow m_{a c}, \\
n_{a c d} \circ\left(n_{a b c} \otimes \mathrm{id}_{m_{c d}}\right) \circ \mathrm{a}_{m_{a b}, m_{b c}, m_{c d}}^{-1} & =n_{a b d} \circ\left(\operatorname{id}_{m_{a b}} \otimes n_{b c d}\right),
\end{aligned}
$$

while the coboundary conditions and modifications are given by

$$
\begin{aligned}
m_{a}: \tilde{e}_{a} \rightarrow & e_{a} \\
n_{a b}: m_{a b} \otimes m_{b} \Rightarrow & m_{a} \otimes \tilde{m}_{a b}, \\
n_{a c} \circ\left(n_{a b c} \otimes \mathrm{id}_{m_{c}}\right)= & \left(\mathrm{id}_{m_{a}} \otimes \tilde{n}_{a b c}\right) \circ \mathrm{a}_{m_{a}, \tilde{m}_{a b}, \tilde{m}_{b c}} \circ\left(n_{a b} \otimes \mathrm{id}_{\tilde{m}_{b c}}\right) \circ \\
& \circ \mathrm{a}_{m_{a b}, m_{b}, \tilde{m}_{b c}}^{-1} \circ\left(\mathrm{id}_{m_{a b}} \otimes n_{b c}\right) \circ \mathrm{a}_{m_{a b}, m_{b c}, m_{c}}
\end{aligned}
$$

and

$$
\begin{aligned}
o_{a}: m_{a} & \Rightarrow \tilde{m}_{a} \\
\tilde{n}_{a b} \circ\left(\mathrm{id}_{m_{a b}} \otimes o_{b}\right) & =\left(o_{a} \otimes \operatorname{id}_{\tilde{m}_{a b}}\right) \circ n_{a b},
\end{aligned}
$$

respectively. 
Remark 3.21. A trivial semistrict principal 2-bundle is described by transition functions $\left(\left\{m_{a b}\right\},\left\{n_{a b c}\right\}\right)$ given in terms of coboundary data $\left(\left\{m_{a}\right\},\left\{n_{a b}\right\}\right)$ according to

$$
\begin{aligned}
m_{a}: \tilde{e}_{a} & \rightarrow e_{a} \quad \text { and } \quad n_{a b}: m_{a b} \otimes m_{b} \Rightarrow m_{a}, \\
n_{a c} \circ\left(n_{a b c} \otimes \mathrm{id}_{m_{c}}\right) & =n_{a b} \circ\left(\operatorname{id}_{m_{a b}} \otimes n_{b c}\right) \circ \mathrm{a}_{m_{a b}, m_{b c}, m_{c}},
\end{aligned}
$$

where $n_{a a}=\mathrm{id}_{m_{a}}$.

To recover principal 2-bundles based on crossed modules as discussed in most of the current literature, we define the following.

Definition 3.22. A strict principal 2-bundle is a weak principal 2-bundle with strict structure 2-group.

A well-known result is then the following.

Proposition 3.23. A strict principal 2-bundle $\Phi$ with strict structure 2-group $\mathscr{G}$ can be equivalently described in terms of Čech cochains taking values in the equivalent crossed module of Lie groups $(\mathrm{H} \stackrel{\partial}{\rightarrow} \mathrm{G}, \triangleright)$. In particular, we have a $\mathrm{G}$-valued $\check{C}$ ech 1-cochain $\left\{g_{a b}\right\}$ and an $\mathrm{H}$-valued $\check{C}$ ech 2-cochain $\left\{h_{a b c}\right\}$ such that

$$
\partial\left(h_{a b c}\right) g_{a b} g_{b c}=g_{a c} \quad \text { and } \quad h_{a c d} h_{a b c}=h_{a b d}\left(g_{a b} \triangleright h_{b c d}\right) .
$$

Coboundaries are then described in terms of G-valued Čech 0-cochains $\left\{g_{a}\right\}$ and $\mathrm{H}$ valued $\check{C}$ ech 1-cochains $\left\{h_{a b}\right\}$. In particular, any two strict principal 2-bundles $\left(\left\{g_{a b}\right\}\right.$, $\left.\left\{h_{a b c}\right\}\right)$ and $\left(\left\{\tilde{g}_{a b}\right\},\left\{\tilde{h}_{a b c}\right\}\right)$ are said to equivalent if and only if

$$
g_{a} \tilde{g}_{a b}=\partial\left(h_{a b}\right) g_{a b} g_{b} \quad \text { and } \quad h_{a c} h_{a b c}=\left(g_{a} \triangleright \tilde{h}_{a b c}\right) h_{a b}\left(g_{a b} \triangleright h_{b c}\right) .
$$

In addition, any two coboundaries $\left(\left\{g_{a}\right\},\left\{h_{a b}\right\}\right)$ and $\left(\left\{\tilde{g}_{a}\right\},\left\{\tilde{h}_{a b}\right\}\right)$ are equivalent if and only if there is an $\mathrm{H}$-valued $\check{C}$ ech 0 -cochain $\left\{h_{a}\right\}$ such that

$$
\tilde{g}_{a}=g_{a} \partial\left(h_{a}\right) \quad \text { and } \quad \tilde{h}_{a b}=\left(g_{a} \triangleright h_{a} h_{b}^{-1}\right) h_{a b} .
$$

Proof: Let us again sketch the identification. For a strict principal 2-bundle, the cocycle and coboundary conditions, as well as the coherence equation for modifications, reduce to

$$
\begin{aligned}
n_{a b c}: m_{a b} \otimes m_{b c} & \Rightarrow m_{a c}, \\
n_{a c d} \circ\left(n_{a b c} \otimes \operatorname{id}_{m_{c d}}\right) & =n_{a b d} \circ\left(\operatorname{id}_{m_{a b}} \otimes n_{b c d}\right),
\end{aligned}
$$

and

$$
\begin{aligned}
m_{a}: \tilde{e}_{a} & \rightarrow e_{a} \\
n_{a b}: m_{a b} \otimes m_{b} & \Rightarrow m_{a} \otimes \tilde{m}_{a b}, \\
n_{a c} \circ\left(n_{a b c} \otimes \mathrm{id}_{m_{c}}\right) & =\left(\operatorname{id}_{m_{a}} \otimes \tilde{n}_{a b c}\right) \circ\left(n_{a b} \otimes \mathrm{id}_{\tilde{m}_{b c}}\right) \circ\left(\mathrm{id}_{m_{a b}} \otimes n_{b c}\right),
\end{aligned}
$$

and

$$
\begin{aligned}
o_{a}: m_{a} & \Rightarrow \tilde{m}_{a} \\
\tilde{n}_{a b} \circ\left(\operatorname{id}_{m_{a b}} \otimes o_{b}\right) & =\left(o_{a} \otimes \operatorname{id}_{\tilde{m}_{a b}}\right) \circ n_{a b} .
\end{aligned}
$$


Next, recall the identification of strict Lie 2-groups with crossed modules of Lie groups of Proposition 2.36. To go from a crossed module of Lie groups $\mathrm{H} \stackrel{\partial}{\rightarrow} \mathrm{G}$ to a strict Lie 2-group $\mathscr{G}$, we identify $\mathscr{G}$ with $(\mathrm{G}, \mathrm{G} \ltimes \mathrm{H})$ in terms of the Lie groups $\mathrm{G}$ and $\mathrm{H}$ contained in the equivalent crossed module, we can identify $m_{a b}=g_{a b}$ and $n_{a b c}=\left(g_{a b c}, h_{a b c}\right)$. From

$$
\begin{aligned}
g_{a b c} & =\mathrm{t}\left(n_{a b c}\right)=g_{a c}=m_{a c}, \\
\mathrm{~s}\left(n_{a b c}\right) & =m_{a b} \otimes m_{b c}=g_{a b} g_{b c}=\partial\left(h_{a b c}^{-1}\right) g_{a b c},
\end{aligned}
$$

we immediately obtain the first equation in (3.28a). Likewise, using $\operatorname{id}_{m_{a b}}=\left(g_{a b}, \mathbb{1}_{\mathbf{H}}\right)$ and (2.19), it is a straightforward exercise to show that (3.29a) simplifies to the second equation in (3.28a). We skip the inverse transition from Lie 2-groups to crossed modules here; details on this point can be found in the proof of Proposition 4.30.

Following the same line of arguments, the coboundary conditions (3.29b) and modifications $(3.29 \mathrm{c})$ are rewritten as $(3.28 \mathrm{~b})$ and $(3.28 \mathrm{c})$.

Remark 3.24. In the strict setting, we may define

$$
\ell_{a b}:=n_{a b} \otimes \mathrm{id}_{\bar{m}_{b}},
$$

where $m \otimes \bar{m}=\mathrm{id}_{e}$. It is easy to see that $\ell_{a b}: m_{a b} \Rightarrow m_{a} \otimes \tilde{m}_{a b} \otimes \bar{m}_{b}$, and, in particular, if the bundle is trivial, then $\ell_{a b}: m_{a b} \Rightarrow m_{a} \otimes \bar{m}_{b}$. In this case, one may show that $n_{a b c}$ can be rewritten in terms of $\ell_{a b}$ as

$$
n_{a b c}=\ell_{a c}^{-1} \circ\left(\ell_{a b} \otimes \ell_{b c}\right)
$$

It is amusing to note the resemblance with a trivial Abelian gerbe: the only difference is that ordinary products are replaced by $\circ$ and $\otimes$.

\section{Differentiating semistrict Lie 2-groups}

In order to define connective structures on semistrict principal 2-bundles, we first need to develop a way of differentiating a semistrict Lie 2-group to a semistrict Lie 2-algebra. The approach we shall develop is based on an idea of Ševera's [23] (see also Jurčo [39]).

As before, we let $X$ be a smooth manifold. The sheaf of smooth differential $p$-forms on $X$ is denoted by $\Omega_{X}^{p}$, and we set $\Omega_{X}^{\bullet}:=\bigoplus_{p \geq 0} \Omega_{X}^{p}$. In general, given a module $\mathfrak{v}=\bigoplus_{k \in \mathbb{Z}} \mathfrak{v}_{k}$ with a $\mathbb{Z}$-grading, one may always introduce a $\mathbb{Z}_{2}$-grading referred to as the Graßmann parity in terms of the parity of degrees: $\mathfrak{v}=\bigoplus_{k \in \mathbb{Z}} \mathfrak{v}_{2 k} \oplus \bigoplus_{k \in \mathbb{Z}} \mathfrak{v}_{2 k-1}$. Elements of $\bigoplus_{k \in \mathbb{Z}} \mathfrak{v}_{2 k}$ are said to be Graßmann-even while elements of $\bigoplus_{k \in \mathbb{Z}} \mathfrak{v}_{2 k-1}$ are said to be Graßmannodd, respectively. We shall also make use of the Graßmann-parity changing functor $\Pi$. For instance, $\mathbb{R}^{m \mid n}:=\mathbb{R}^{m} \oplus \Pi \mathbb{R}^{n}$. Moreover, $\mathfrak{v}[k]$ will denote the module $\mathfrak{v}$ with grading shifted by $k$. Similarly, $T[k] X$ denotes the tangent bundle of $X$ with the grading of the fibres shifted by $k$. 


\subsection{Basic ideas}

Definition 4.1. Let $\sigma: Y \rightarrow X$ be a surjective submersion and $\mathrm{G}$ be a Lie group. $A$ G-valued descent datum on $\sigma: Y \rightarrow X$ is a map $g: Y \times_{X} Y \rightarrow \mathrm{G}$ such that ${ }^{11}$

$$
g\left(x_{1}, x_{1}\right)=\mathbb{1}_{\mathrm{G}} \quad \text { and } \quad g\left(x_{1}, x_{2}\right) g\left(x_{2}, x_{3}\right)=g\left(x_{1}, x_{3}\right)
$$

for all $\left(x_{1}, x_{2}, x_{3}\right) \in Y \times_{X} Y \times_{X} Y$.

Specifically, a given descent datum describes the descent of a trivial principal G-bundle over $Y$ to a non-trivial principal G-bundle over $X$. The following example makes this more transparent.

Example 4.2. Let $X$ be a smooth manifold with covering $\mathfrak{U}=\left\{U_{a}\right\}_{a \in \mathcal{I}}$ indexed by the index set $\mathcal{I}$. Consider the trivial projection $\sigma: \mathcal{I} \times X \rightarrow X$. A G-valued descent datum is then given by a map $g: \mathcal{I} \times \mathcal{I} \times X \rightarrow X$ such that $g(a, a, x)=\mathbb{1}_{\mathrm{G}}$ and $g(a, b, x) g(b, c, x)=g(a, c, x)$ for all $a, b \in \mathcal{I}$ and $x \in X$. Setting $g_{a b}(x):=g(a, b, x)$, we have obtained $a$ G-valued Čech 1 cocycle $\left\{g_{a b}\right\}$ on $X$ relative to the covering $\mathfrak{U}$. This, in turn, describes a principal G-bundle over $X$.

Below, we shall be interested in the trivial projection $\sigma: \mathbb{R}^{0 \mid 1} \times X \rightarrow X$, so a G-valued decent datum is in this case given by a map $g: \mathbb{R}^{0 \mid 1} \times \mathbb{R}^{0 \mid 1} \times X \rightarrow \mathrm{G}$ such that

$$
g\left(\theta_{0}, \theta_{1}, x\right) g\left(\theta_{1}, \theta_{2}, x\right)=g\left(\theta_{0}, \theta_{2}, x\right) \quad \text { for } \quad x \in X .
$$

We can regard the maps from the surjective submersion $\mathbb{R}^{0 \mid 1} \times X \rightarrow X$ to a descent datum as a contravariant functor from the category of smooth manifolds to the category of sets. As we shall see below, this functor is representable by $\mathfrak{g}[-1]$, where $\mathfrak{g}$ is the Lie algebra of G. In particular, calculating the moduli of this functor yields the Lie algebra $\mathfrak{g}$ as a vector space. To describe its Lie bracket, one needs to compute the action of its ChevalleyEilenberg differential ${ }^{12} \mathrm{~d}_{\mathrm{CE}}$. This differential is governed by a generator of the natural action of $C^{\infty}\left(\mathbb{R}^{0 \mid 1}, \mathbb{R}^{0,1}\right)$ on the descent data, as was first discussed by Kontsevich [40] (see also [23]). Let us now review this in some more detail.

Proposition 4.3. There is a natural isomorphism $H^{0}\left(X, \Omega_{X}^{\bullet}\right) \cong C^{\infty}\left(C^{\infty}\left(\mathbb{R}^{0 \mid 1}, X\right), \mathbb{R}\right)$.

Proof. Consider first the case $X=\mathbb{R}^{n}$ equipped with standard coordinates $\left(x^{1}, \ldots, x^{n}\right)$. An element of $C^{\infty}\left(\mathbb{R}^{0 \mid 1}, \mathbb{R}^{n}\right)$ is parameterised as $\left(x^{1}, \ldots, x^{n}\right)=\left(a^{1}+\alpha^{1} \theta, \ldots, a^{n}+\alpha^{n} \theta\right)$, where $\theta, \alpha^{i} \in \mathbb{R}^{0 \mid 1}$ are Graßmann-odd and $a^{i} \in \mathbb{R}$ are Graßmann-even for $i=1, \ldots, n$. We thus have established $C^{\infty}\left(\mathbb{R}^{0 \mid 1}, \mathbb{R}^{n}\right) \cong \mathbb{R}^{n \mid n}$. Furthermore, functions on $\mathbb{R}^{n \mid n}$ are polynomials in the Graßmann-odd coordinates. Thus, upon identifying the $a^{i}$ with the coordinates on $\mathbb{R}^{n}$ and the $\alpha^{i}$ with the corresponding differential 1-forms, we have obtained $H^{0}\left(\mathbb{R}^{n}, \Omega_{\mathbb{R}^{n}}\right) \cong$ $C^{\infty}\left(C^{\infty}\left(\mathbb{R}^{0 \mid 1}, \mathbb{R}^{n}\right), \mathbb{R}\right)$. For a general smooth manifold $X$, we have thus a local isomorphism between $C^{\infty}\left(\mathbb{R}^{0 \mid 1}, X\right)$ and $T[-1] X$. However, this isomorphism is independent of the choice of coordinates, and, hence, $C^{\infty}\left(\mathbb{R}^{0 \mid 1}, X\right)$ and $T[-1] X$ can be naturally identified. This, in turn, leads to the isomorphism $H^{0}\left(X, \Omega_{X}^{\bullet} \cong C^{\infty}\left(C^{\infty}\left(\mathbb{R}^{0 \mid 1}, X\right), \mathbb{R}\right)\right.$.

\footnotetext{
${ }^{11}$ Recall that $\left.Y \times_{X} \cdots \times_{X} Y:=\left\{\left(x_{1}, \ldots, x_{k}\right)\right) \in Y \times \cdots \times Y \mid \sigma\left(x_{1}\right)=\cdots=\sigma\left(x_{k}\right)\right\}$.

${ }^{12}$ See appendix A for the relevant definitions.
} 
Furthermore, the de Rham differential d on $H^{0}\left(X, \Omega_{X}^{\bullet}\right)$ follows from the action of $C^{\infty}\left(\mathbb{R}^{0 \mid 1}, \mathbb{R}^{0 \mid 1}\right)$ on $C^{\infty}\left(\mathbb{R}^{0 \mid 1}, X\right)$. Concretely, transformations of the form $\theta \mapsto \tilde{\theta}=b \theta+\beta$ for $b \in \mathbb{R}, \beta \in \mathbb{R}^{0 \mid 1}$ induce an action on elements of $C^{\infty}\left(\mathbb{R}^{0 \mid 1}, X\right)$ which in local coordinates $\left(x^{1}, \ldots, x^{n}\right)$ of $X$ is given by

$$
x^{i}(\theta)=a^{i}+\alpha^{i} \theta \quad \mapsto \quad x^{i}(\tilde{\theta})=a^{i}+(b \theta+\beta) \alpha^{i}=\left(a^{i}+\beta \alpha^{i}\right)+b \alpha^{i} \theta
$$

for $i=1, \ldots, n$. Translated into differential forms, this means that $x^{i} \mapsto x^{i}+\beta \mathrm{d} x^{i}$ and $\mathrm{d} x^{i} \mapsto b \mathrm{~d} x^{i}$. We thus arrive at the following result.

Proposition 4.4. The action of the de Rham differential $\mathrm{d}$ on $H^{0}\left(X, \Omega_{X}^{\bullet}\right)$ translates to an action of the generator $\mathrm{d}_{\mathrm{K}}$ of $C^{\infty}\left(\mathbb{R}^{0 \mid 1}, \mathbb{R}^{0 \mid 1}\right)$ given by

$$
\mathrm{d}_{\mathrm{K}} f(x(\theta))=\frac{\mathrm{d}}{\mathrm{d} \varepsilon} f(x(\theta+\varepsilon))
$$

for any $f \in C^{\infty}\left(C^{\infty}\left(\mathbb{R}^{0 \mid 1}, X\right), \mathbb{R}\right)$.

We would like to point out that the differential $\mathrm{d}_{\mathrm{K}}$ extends to smooth functions $f \in$ $C^{\infty}\left(C^{\infty}\left(\mathbb{R}^{0 \mid k}, X\right), \mathbb{R}\right)$, since there is a natural action of $C^{\infty}\left(\mathbb{R}^{0 \mid 1}, \mathbb{R}^{0 \mid 1}\right)$ on $C^{\infty}\left(\mathbb{R}^{0 \mid k}, X\right)$. Specifically, its action on a function of several Graßmann-odd coordinates $\left(\theta_{0}, \ldots, \theta_{k-1}\right)$ is diagonal,

$$
\mathrm{d}_{\mathrm{K}} f\left(x\left(\theta_{0}, \ldots, \theta_{k-1}\right)\right)=\frac{\mathrm{d}}{\mathrm{d} \varepsilon} f\left(x\left(\theta_{0}+\varepsilon, \ldots, \theta_{k-1}+\varepsilon\right)\right),
$$

where $x\left(\theta_{0}, \ldots, \theta_{k-1}\right):=\left(x^{1}\left(\theta_{0}, \ldots, \theta_{k-1}\right), \ldots, x^{n}\left(\theta_{0}, \ldots, \theta_{k-1}\right)\right)$.

Example 4.5. Consider a Graßmann-even function $f\left(x\left(\theta_{0}, \theta_{1}\right)\right)=g+\phi \theta_{0}+\psi \theta_{1}+F \theta_{0} \theta_{1}$. We obtain

$$
\mathrm{d}_{\mathrm{K}} f\left(x\left(\theta_{0}, \theta_{1}\right)\right)=\frac{\mathrm{d}}{\mathrm{d} \varepsilon} f\left(x\left(\theta_{0}+\varepsilon, \theta_{1}+\varepsilon\right)\right)=-\phi-\psi+\left(\theta_{0}-\theta_{1}\right) F
$$

from (4.5). Comparing coefficients in the Graßmann-odd coordinates, we can read off the action of an induced operator, again denoted by $\mathrm{d}_{\mathrm{K}}$ on the components

$$
\mathrm{d}_{\mathrm{K}} g=-\phi-\psi, \quad \mathrm{d}_{\mathrm{K}} \phi=F, \quad \mathrm{~d}_{\mathrm{K}} \psi=-F, \quad \text { and } \quad \mathrm{d}_{\mathrm{K}} F=0 .
$$

Proposition 4.6. The operator $\mathrm{d}_{\mathrm{K}}$ is a differential. That is, it has the following properties:

(i) $\mathrm{d}_{\mathrm{K}} \circ \mathrm{d}_{\mathrm{K}}=0$,

(ii) for any $f \in C^{\infty}\left(C^{\infty}\left(\mathbb{R}^{0 \mid k}, X\right), \mathbb{R}\right)$ and $g \in C^{\infty}\left(C^{\infty}\left(\mathbb{R}^{0 \mid l}, X\right), \mathbb{R}\right)$, the operator $\mathrm{d}_{\mathrm{K}}$ obeys a graded Leibniz rule,

$$
\mathrm{d}_{\mathrm{K}}(f g)=\left(\mathrm{d}_{\mathrm{K}} f\right) g+(-1)^{|f|} f \mathrm{~d}_{\mathrm{K}} g,
$$

where $|f|$ denotes the Graßmann parity of $f$.

Proof. These properties are an immediate consequence of the definition of $\mathrm{d}_{\mathrm{K}}$. 


\subsection{Lie algebra of a Lie group}

Having collected all relevant ideas, let us put them to use and start by computing the Lie algebra of a Lie group as a guiding example for the case of Lie 2-groups. This has been done in $[23,39]$, and our discussion below is an expanded version of the one found in these references.

Consider a Lie group $\mathrm{G}$ with Lie algebra $\mathfrak{g}=T_{\mathbb{1}_{\mathrm{G}}} \mathrm{G}$. To prepare our discussion for semistrict Lie 2-groups, we shall not assume that $G$ is a matrix group, rather we only make use of the fact that there is a local diffeomorphism $\varphi$ between a neighbourhood $U_{\mathfrak{g}}$ of $0 \in \mathfrak{g}$ and a neighbourhood $U_{\mathrm{G}}$ of $\mathbb{1}_{\mathrm{G}} \in \mathrm{G}$ with $\varphi(a)=g$ for $a \in U_{\mathfrak{g}}$ and $g \in$ $U_{\mathrm{G}}, \varphi(0)=\mathbb{1}_{\mathrm{G}}$, and $\left.\varphi_{*}\right|_{0}$ is the identity. In addition, we wish to restrict ourselves to infinitesimal neighbourhoods by considering elements of $\mathfrak{g}[-1]$ multiplied by a Graßmannodd coordinate.

Proposition 4.7. Let $\varphi: U_{\mathfrak{g}} \rightarrow U_{\mathrm{G}}$ be the above-described local diffeomorphism. For $a, a_{1,2} \in \mathfrak{g}[-1]$, we have the following relations:

$$
\begin{aligned}
\varphi(a \theta)^{-1} & =\varphi(-a \theta), \\
\varphi^{-1}\left(\varphi\left(a_{1} \theta_{1}\right) \varphi\left(a_{2} \theta_{2}\right)\right) & =a_{1} \theta_{1}+a_{2} \theta_{2}-a_{1} \cdot a_{2} \theta_{1} \theta_{2},
\end{aligned}
$$

where the operation $: \mathfrak{g}[-1] \times \mathfrak{g}[-1] \rightarrow \mathfrak{g}[-2]$ is defined by the second equation. This operation is bilinear and $a_{1} \cdot a_{2}+a_{2} \cdot a_{1}=\left[a_{1}, a_{2}\right]$ is the Lie bracket shifted by one degree.

Proof. First of all, it is clear that $\varphi^{-1}\left(\varphi\left(a_{1} \theta_{1}\right) \varphi\left(a_{2} \theta_{2}\right)\right)$ is a polynomial in the Graßmannodd coordinates. The terms of this expression linear in $\theta_{1}$ and $\theta_{2}$ then follow from putting $\theta_{1}$ or $\theta_{2}$ to zero, respectively. In the special case when $\theta_{1}=\theta_{2}$ and $a_{1}=-a_{2}$, we recover the first equation of (4.9). It remains to understand the operation ' $\because$. For this, consider the expression

$$
\begin{aligned}
& \varphi^{-1}\left(\varphi\left(a_{1} \theta_{1}+a_{2} \theta_{2}\right) \varphi\left(a_{3} \theta_{3}+a_{4} \theta_{4}\right)\right)=a_{1} \theta_{1}+\cdots+a_{4} \theta_{4}- \\
& \quad-a_{1} \cdot a_{3} \theta_{1} \theta_{3}-a_{1} \cdot a_{4} \theta_{1} \theta_{4}-a_{2} \cdot a_{3} \theta_{2} \theta_{3}-a_{2} \cdot a_{4} \theta_{2} \theta_{4}+\mathcal{O}\left(\theta^{3}\right) .
\end{aligned}
$$

This expansion follows from the second equation of (4.9) and the special cases $a_{1}=a_{3}=0$, $a_{1}=a_{4}=0, a_{2}=a_{3}=0$, and $a_{2}=a_{4}=0$. Bilinearity of '. ' then follows directly from this expression for $\theta_{1}=\theta_{2}$ and $\theta_{3}=\theta_{4}$ together with the second equation of (4.9). Furthermore, considering the algebra element corresponding to the group commutator

$$
\varphi^{-1}\left(\varphi\left(-a_{1} \theta_{1}\right) \varphi\left(-a_{2} \theta_{2}\right) \varphi\left(a_{1} \theta_{1}\right) \varphi\left(a_{2} \theta_{2}\right)\right)=\left(a_{1} \cdot a_{2}+a_{2} \cdot a_{1}\right) \theta_{1} \theta_{2},
$$

where the expansion follows from considering the cases either $a_{1}$ and/or $a_{2}$ vanish, we find the shifted Lie bracket $\left[a_{1}, a_{2}\right]=a_{1} \cdot a_{2}+a_{2} \cdot a_{1}$. This concludes the proof.

Remark 4.8. For matrix Lie groups, we may suggestively write

$$
\begin{aligned}
\left(\mathbb{1}_{\mathrm{G}}+a \theta\right)^{-1} & =\mathbb{1}_{\mathrm{G}}-a \theta, \\
\left(\mathbb{1}_{\mathrm{G}}+a_{1} \theta_{1}\right)\left(\mathbb{1}_{\mathrm{G}}+a_{2} \theta_{2}\right) & =\mathbb{1}_{\mathrm{G}}+a_{1} \theta_{1}+a_{2} \theta_{2}-a_{1} \cdot a_{2} \theta_{1} \theta_{2} .
\end{aligned}
$$

In addition, one may also define products between elements $g$ and $a$ of $\mathrm{G}$ and $\mathfrak{g}[-1]$, respectively. For matrix Lie groups, we simply write ga. For general Lie groups, one replaces such expressions by the pullback $\mathrm{L}_{g}^{*} a$ of $a$, where $\mathrm{L}_{g}$ denotes left multiplication on $\mathrm{G}$. 
We are now ready to discuss the computation of the Lie algebra of a Lie group by Ševera's construction [23]. Consider a G-valued descent datum on the trivial projection $\mathbb{R}^{0 \mid 1} \times X \rightarrow X$. That is, we a have smooth map $g: \mathbb{R}^{0 \mid 1} \times \mathbb{R}^{0 \mid 1} \times X \rightarrow \mathrm{G}$ satisfying the cocycle condition (4.2). Since we are interested in the functor from the category of smooth manifolds to the category of descent data in the following, we shall suppress the explicit dependence on $x \in X$ and simply write $\left\{g_{01}:=g\left(\theta_{0}, \theta_{1}\right)\right\}$ with $g_{01} g_{12}=g_{02}$ and $g_{10}=g_{01}^{-1}$. Then, we have the following result.

Lemma 4.9. Letting $g(\theta):=g(\theta, 0)$, we have

$$
g\left(\theta_{0}, \theta_{1}\right)=g\left(\theta_{0}\right) g\left(\theta_{1}\right)^{-1}
$$

Proof. This is an immediate consequence of (4.2).

Next, we may expand ${ }^{13} g\left(\theta_{0}\right)=\mathbb{1}_{\mathrm{G}}+a \theta_{0}$ for some $a \in \mathfrak{g}[-1]$ since $g(0)=g(0,0)=\mathbb{1}_{\mathrm{G}}$. Together with the Propositions 4.4 and 4.7 , we get the following result.

Proposition 4.10. A G-valued descent datum on $\mathbb{R}^{0 \mid 1} \times X \rightarrow X$ is parametrised by an element $a \in \mathfrak{g}[-1]$ according to

$$
g_{01}=\left(\mathbb{1}_{\mathrm{G}}+a \theta_{0}\right)\left(\mathbb{1}_{\mathrm{G}}-a \theta_{1}\right)=\mathbb{1}_{\mathrm{G}}+a\left(\theta_{0}-\theta_{1}\right)+\frac{1}{2}[a, a] \theta_{0} \theta_{1} .
$$

The induced differential is given by

$$
\mathrm{d}_{\mathrm{K}} a+\frac{1}{2}[a, a]=0 .
$$

As stated previously, we wish to identify the induced action of the differential $d_{K}$ with the Chevalley-Eilenberg differential $d_{\mathrm{CE}}$ on $\mathfrak{g}$. Recall that the Chevalley-Eilenberg differential of a Lie algebra $\mathfrak{g}$ acts as

$$
\mathrm{d}_{\mathrm{CE}} \check{\tau}^{i}=-\frac{1}{2} f_{j k}^{i} \check{\tau}^{j} \wedge \check{\tau}^{k}
$$

on basis elements $\tilde{\tau}^{i}$ of the dual Lie algebra $\mathfrak{g}^{\vee}$ of $\mathfrak{g}$. Here, the $f_{j k}^{i}$ are the structure constants of $\mathfrak{g}$ with respect to the basis elements $\tau_{i}$ of $\mathfrak{g}$ with $\check{\tau}^{i}\left(\tau_{j}\right)=\delta_{j}^{i}$. The equation (4.15) amounts to the Maurer-Cartan equation $\mathrm{d}_{\mathrm{CE}} a+\frac{1}{2}[a, a]=0$ which should be regarded as the equation (4.16) evaluated for a polynomial in $a^{i}$ with $a=a^{i} \tau_{i}$.

Altogether, we have proved the following theorem.

Theorem 4.11. The functor from the category of smooth manifolds $X$ to the category of $\mathrm{G}$-valued descent data on surjective submersions $\mathbb{R}^{0 \mid 1} \times X \rightarrow X$ is parameterised by elements of $\mathfrak{g}[-1]$ with $\mathfrak{g}=\mathrm{Lie}(\mathrm{G})$. The action of the differential $\mathrm{d}_{\mathrm{K}}$ on descent data yields the action of the Chevalley-Eilenberg differential corresponding to $\mathfrak{g}$.

\footnotetext{
${ }^{13}$ To simplify notation, we use suggestive notation for matrix groups, which is readily translated to general expressions involving the diffeomorphism $\varphi$.
} 
Finally, let us consider Čech coboundary transformations on $\left\{g_{01}=g\left(\theta_{0}, \theta_{1}\right)\right\}$. Such transformations are parameterised by smooth maps $p: \mathbb{R}^{0 \mid 1} \rightarrow \mathrm{G}$ with $\left\{p_{0}:=p\left(\theta_{0}\right)\right\}$ and $p(\theta)=p+\pi \theta$ for some $p \in \mathrm{G}$ and $\pi \in T_{p}[-1] \mathrm{G}$ according to

$$
\tilde{g}_{01}=p_{0} g_{01} p_{1}^{-1}=\mathbb{1}_{\mathrm{G}}+\tilde{a}\left(\theta_{0}-\theta_{1}\right)+\frac{1}{2}[\tilde{a}, \tilde{a}] \theta_{0} \theta_{1}
$$

where

$$
\tilde{a}:=p a p^{-1}+\pi p^{-1} \text {. }
$$

Together with the induced differential $\mathrm{d}_{\mathrm{K}} p=-\pi$, we obtain the following.

Proposition 4.12. Consider two equivalent G-valued descent data that are parametrised by $a \in \mathfrak{g}[-1]$ and $\tilde{a} \in \mathfrak{g}[-1]$, respectively. Then there is a Čech coboundary transformations between these, which is parametrised by $p: \mathbb{R}^{0 \mid 1} \rightarrow \mathrm{G}$ with $p(\theta)=p+\pi \theta$ for some $p \in \mathrm{G}$ and $\pi \in T_{p}[-1] \mathrm{G}$, such that

$$
\tilde{a}=p a p^{-1}+\pi p^{-1}=p a p^{-1}+p \mathrm{~d}_{\mathrm{K}} p^{-1} .
$$

The equation $\mathrm{d}_{\mathrm{K}} a+\frac{1}{2}[a, a]=0$ is invariant under coboundary transformations. That is, whenever $\mathrm{d}_{\mathrm{K}} a+\frac{1}{2}[a, a]=0$ we have $\mathrm{d}_{\mathrm{K}} \tilde{a}+\frac{1}{2}[\tilde{a}, \tilde{a}]=0$ and vice versa.

Remark 4.13. Note that by replacing $\mathrm{d}_{\mathrm{K}}$ by the de Rham differential in all of the above, we recover the definition of the curvature of a connection 1-form on a principal bundle with structure group $\mathrm{G}$ as well as its gauge transformation. We will make use of this observation later on.

\subsection{Semistrict Lie 2-algebra of a semistrict Lie 2-group}

Now we generalise the previous discussion to the case of semistrict Lie 2-groups $\mathscr{G}=$ $(M, N)$, which we shall regard as a weak Lie 2-groupoid $\mathrm{B} \mathscr{G}(\{e\}, M, N)$ in the following. In this case, the local diffeomorphism $\varphi=\left(\varphi_{M}, \varphi_{N}\right)$ goes between neighbourhoods $U_{\mathfrak{m}}$ of $\mathfrak{m}:=T_{\mathrm{id}_{e}} M$ and $U_{\mathfrak{n}}$ of $\mathfrak{n}:=T_{\operatorname{id}_{\mathrm{id}_{e}}} N$ as well as neighbourhoods $U_{M}$ of $\operatorname{id}_{e}$ and $U_{N}$ of $\mathrm{id}_{\mathrm{id}_{e}}$. As before, $\varphi(0)=\left(\mathrm{id}_{e}, \mathrm{id}_{\mathrm{id}_{e}}\right)$ and $\left.\varphi_{*}\right|_{0}$ is the identity. Following our previous discussion, we shall again be interested in infinitesimal neighbourhoods and we shall always write suggestively $\mathrm{id}_{e}+a \theta$ and $\operatorname{id}_{\mathrm{id}_{e}}+b \theta$ for $\varphi_{M}(a \theta)$ and $\varphi_{N}(b \theta)$, where $a \in \mathfrak{m}[-1]$ and $b \in \mathfrak{n}[-1]$.

Proposition 4.14. The bifunctor $\otimes: \mathrm{B} \mathscr{G} \times \mathrm{B} \mathscr{G} \rightarrow \mathrm{B} \mathscr{G}$ induces bilinear non-associative products $\otimes: \mathfrak{m}[-1] \times \mathfrak{m}[-1] \rightarrow \mathfrak{m}[-2]$ and $\otimes: \mathfrak{n}[-1] \times \mathfrak{n}[-1] \rightarrow \mathfrak{n}[-2]$ by means of

$$
\begin{aligned}
\left(\mathrm{id}_{e}+a_{1} \theta_{1}\right) \otimes\left(\mathrm{id}_{e}+a_{2} \theta_{2}\right) & =\mathrm{id}_{e}+a_{1} \theta_{1}+a_{2} \theta_{2}-a_{1} \otimes a_{2} \theta_{1} \theta_{2}, \\
\left(\mathrm{id}_{\mathrm{id}_{e}}+b_{1} \theta_{1}\right) \otimes\left(\mathrm{id}_{\mathrm{id}_{e}}+b_{2} \theta_{2}\right) & =\mathrm{id}_{\mathrm{id}_{e}}+b_{1} \theta_{1}+b_{2} \theta_{2}-b_{1} \otimes b_{2} \theta_{1} \theta_{2},
\end{aligned}
$$

where $a_{1,2} \in \mathfrak{m}[-1]$ and $b_{1,2} \in \mathfrak{n}[-1]$, respectively.

Proof. The proof is essentially the same as the one given for Proposition 4.7. 
We now turn to the maps induced by the structure maps $s, t$, and id on $\mathfrak{n}[-1]$ and $\mathfrak{m}[-1]$. Note that for elements $a \in \mathfrak{m}[-1]$ and $b \in \mathfrak{n}[-1]$, we have

$$
\begin{aligned}
\operatorname{id}_{\mathrm{id}_{e}+a \theta} & =\mathrm{id}_{\mathrm{id}_{e}}+\mathrm{id}_{*}(a) \theta, \\
\mathbf{s}\left(\mathrm{idid}_{e}+b \theta\right) & =\mathrm{id}_{e}+\mathrm{s}_{*}(b) \theta, \quad \mathrm{t}\left(\mathrm{id}_{\mathrm{id}_{e}}+b \theta\right)=\mathrm{id}_{e}+\mathrm{t}_{*}(b) \theta,
\end{aligned}
$$

where the differentials are to be taken at $\mathrm{id}_{\mathrm{id}_{e}}$ and $\mathrm{id}_{e}$, respectively. More generally, the following result holds.

Proposition 4.15. Around $\mathrm{id}_{e}+a_{1} \theta_{1}+a_{2} \theta_{2}$ and $\operatorname{id}_{\mathrm{id}_{e}}+b_{1} \theta_{1}+b_{2} \theta_{2}$ for some $a_{1,2} \in \mathfrak{m}[-1]$ and $b_{1,2} \in \mathfrak{n}[-1]$, the structure maps expand as follows:

$$
\begin{aligned}
& \begin{aligned}
\mathrm{id}_{\mathrm{id}_{e}+a_{1}} \theta_{1}+a_{2} \theta_{2} & = \\
& =\mathrm{id}_{\mathrm{id}_{e}}+\mathrm{id}_{*}\left(a_{1}\right) \theta_{1}+\mathrm{id}_{*}\left(a_{2}\right) \theta_{2}+\left(\mathrm{id}_{*}\left(a_{1} \otimes a_{2}\right)-\mathrm{id}_{*}\left(a_{1}\right) \otimes \mathrm{id}_{*}\left(a_{2}\right)\right) \theta_{1} \theta_{2}, \\
\mathrm{~s}\left(\mathrm{id}_{\mathrm{id}_{e}}\right. & \left.+b_{1} \theta_{1}+b_{2} \theta_{2}\right)= \\
& =\mathrm{id}_{e}+\mathrm{s}_{*}\left(b_{1}\right) \theta_{1}+\mathrm{s}_{*}\left(b_{2}\right) \theta_{2}+\left(\mathbf{s}_{*}\left(b_{1} \otimes b_{2}\right)-\mathbf{s}_{*}\left(b_{1}\right) \otimes \mathbf{s}_{*}\left(b_{2}\right)\right) \theta_{1} \theta_{2} \\
\mathrm{t}\left(\mathrm{id}_{\mathrm{id}_{e}}\right. & \left.+b_{1} \theta_{1}+b_{2} \theta_{2}\right)= \\
& =\mathrm{id}_{e}+\mathrm{t}_{*}\left(b_{1}\right) \theta_{1}+\mathrm{t}_{*}\left(b_{2}\right) \theta_{2}+\left(\mathrm{t}_{*}\left(b_{1} \otimes b_{2}\right)-\mathrm{t}_{*}\left(b_{1}\right) \otimes \mathbf{t}_{*}\left(b_{2}\right)\right) \theta_{1} \theta_{2} .
\end{aligned}
\end{aligned}
$$

Proof. The map id is compatible with $\otimes$ on $M$ in the following way:

$$
\operatorname{id}_{\left(\mathrm{id}_{e}+a_{1} \theta_{1}\right) \otimes\left(\mathrm{id}_{e}+a_{2} \theta_{2}\right)}=\mathrm{id}_{\mathrm{id}_{e}+a_{1} \theta_{1}} \otimes \mathrm{id}_{\mathrm{id}_{e}+a_{2} \theta_{2}} .
$$

Expanding both sides of this equation according to Proposition 4.14 yields the desired result. The argument for the maps $s$ and $t$ is fully analogous.

Finally, we have to discuss an induced concatenation map on $\mathfrak{n}[-1]$. Note that if $\mathbf{s}_{*}\left(b_{1}\right)=\mathbf{t}_{*}\left(b_{2}\right)$ for some $b_{1,2} \in \mathfrak{n}[-1]$, then $\mathbf{s}\left(\mathrm{id}_{\mathrm{id}_{e}}+b_{1} \theta\right)=\mathrm{t}\left(\mathrm{id}_{\mathrm{id}_{e}}+b_{2} \theta\right)$.

Definition 4.16. For elements $b_{1,2} \in \mathfrak{n}[-1]$ with $\mathrm{s}_{*}\left(b_{1}\right)=\mathrm{t}_{*}\left(b_{2}\right)$, we define implicitly

$$
\left(\mathrm{id}_{\mathrm{id}_{e}}+b_{1} \theta\right) \circ\left(\mathrm{id}_{\mathrm{id}_{e}}+b_{2} \theta\right)=: \mathrm{id}_{\mathrm{id}_{e}}+b_{1} \circ b_{2} \theta .
$$

It trivially follows that $b_{1} \circ 0=b_{1}$ for $\mathrm{s}_{*}\left(b_{1}\right)=0$ and $0 \circ b_{2}=b_{2}$ for $\mathrm{t}_{*}\left(b_{2}\right)=0$. More generally, the induced concatenation map satisfies the following.

Proposition 4.17. For $b_{1,2,3,4} \in \mathfrak{n}[-1]$ with $\mathrm{s}_{*}\left(b_{1}\right)=\mathrm{t}_{*}\left(b_{3}\right), \mathrm{s}_{*}\left(b_{2}\right)=\mathrm{t}_{*}\left(b_{4}\right)$, and $\mathrm{s}_{*}\left(b_{1} \otimes\right.$ $\left.b_{2}\right)=\mathrm{t}_{*}\left(b_{3} \otimes b_{4}\right)$, we have

$$
\left(\mathrm{id}_{\mathrm{id}_{e}}+b_{1} \theta_{1}+b_{2} \theta_{2}\right) \circ\left(\mathrm{id}_{\mathrm{id}_{e}}+b_{3} \theta_{1}+b_{4} \theta_{2}\right)=\mathrm{id}_{\mathrm{id}_{e}}+b_{1} \circ b_{3} \theta_{1}+b_{2} \circ b_{4} \theta_{2} .
$$

Remark 4.18. Note that above we have linearised all the structure maps $\mathrm{s}, \mathrm{t}$, id, $\otimes$, and $\circ$ at $\mathrm{id}_{e}$ or $\mathrm{id}_{\mathrm{id}_{e}}$ and obtained maps on $\mathfrak{m}[-1]$ or $\mathfrak{n}[-1]$. We can certainly consider linearisations also at other points $p$ of $M$ or $N$, leading to maps on $T_{p}[-1] M$ or $T_{p}[-1] N$. The formulce in these cases are obvious generalisations of the ones derived above. 
Remark 4.19. In the following, we shall simply write $\mathrm{s}, \mathrm{t}$, and $\mathrm{id}$ for $\mathrm{s}_{*}, \mathrm{t}_{*}$, and $\mathrm{id}_{*}$, slightly abusing notation. We shall also write $\operatorname{id}_{a}$ instead of $\operatorname{id}_{*}(a)$. The distinction between these linear maps and the finite maps on $M$ and $N$ should always be clear from the context.

This completes the preliminary discussion, and we can turn to the differentiation of a semistrict Lie 2 -group $\mathscr{G}=(M, N)$ to a 2 -term $L_{\infty}$-algebra. Following our discussion for Lie groups, we consider the functor from the category of smooth manifolds $X$ to the category of $\mathscr{G}$-valued descent data on surjective submersions $\mathbb{R}^{0 \mid 1} \times X \rightarrow X$ that are represented by $M$-valued 1-cells $\left\{m_{01}:=m\left(\theta_{0}, \theta_{1}\right)\right\}$ and $N$-valued 2-cells $\left\{n_{012}:=n\left(\theta_{0}, \theta_{1}, \theta_{2}\right)\right\}$ so that

$$
n_{012}: m_{01} \otimes m_{12} \Rightarrow m_{02},
$$

and

$$
n_{023} \circ\left(n_{012} \otimes \mathrm{id}_{m_{23}}\right)=n_{013} \circ\left(\mathrm{id}_{m_{01}} \otimes n_{123}\right) \circ \mathrm{a}_{m_{01}, m_{12}, m_{23}} .
$$

Analogously to Lemma 4.9, we have the following statement; see also Remark 3.21.

Lemma 4.20. The functor $\left(\left\{m_{01}\right\},\left\{n_{012}\right\}\right)$ is trivialised by the following $\mathscr{G}_{\text {-valued }}$ Cech 1-cochains $\left(\left\{m_{0}\right\},\left\{n_{01}\right\}\right)$ :

$$
m_{0}:=m\left(\theta_{0}\right):=m\left(\theta_{0}, 0\right) \quad \text { and } \quad n_{01}:=n\left(\theta_{0}, \theta_{1}\right):=n\left(\theta_{0}, \theta_{1}, 0\right) .
$$

That is, $n_{01}: m_{01} \otimes m_{1} \Rightarrow m_{0}$ with

$$
n_{02} \circ\left(n_{012} \otimes \mathrm{id}_{m_{2}}\right)=n_{01} \circ\left(\mathrm{id}_{m_{01}} \otimes n_{12}\right) \circ \mathrm{a}_{m_{01}, m_{12}, m_{2}} .
$$

Furthermore,

$$
m(0)=\operatorname{id}_{e} \quad \text { and } \quad n\left(\theta_{0}, 0\right)=\operatorname{id}_{m_{0}} .
$$

Proof. This statement is readily proved by computation and comparison with Remark 3.21. To this end one needs to use the fact that $\mathrm{a}_{m, m^{\prime}, \text { id }_{e}}$ is trivial for all $m, m^{\prime} \in M$; see Proposition 2.28. Equations (4.28) follow from the normalisations of the cocycle conditions for semistrict principal 2-bundles, cf. Lemma 3.13.

Remark 4.21. Clearly, there is a one-to-one correspondence between $\mathscr{G}$-valued descent data $\left(\left\{m_{01}\right\},\left\{n_{012}\right\}\right)$ and trivialising $\mathscr{G}$-valued $\check{C}$ ech 1-cochains $\left(\left\{m_{0}\right\},\left\{n_{01}\right\}\right)$. Moreover, by a modification isomorphism, any trivialising $\mathscr{G}$-valued Cech 1-cochain $\left(\left\{m_{0}\right\},\left\{n_{01}\right\}\right)$ is equivalent to one of the form (4.26).

Proposition 4.22. A descent datum $\left(\left\{m_{01}\right\},\left\{n_{012}\right\}\right)$ and the corresponding coboundary datum $\left(\left\{m_{0}\right\},\left\{n_{01}\right\}\right)$ are parametrised by 1-cells $\alpha \in \mathfrak{m}[-1]$ and 2-cells $\beta \in \mathfrak{n}[-2]$ with

$$
\alpha: 0 \rightarrow 0 \text { and } \beta: \mathrm{s}(\beta) \Rightarrow 0
$$

according to the following expansions in the Graßmann-odd coordinates:

$$
\begin{aligned}
m_{0}= & \operatorname{id}_{e}+\alpha \theta_{0}, \\
n_{01}= & \operatorname{id}_{i_{e}}+\operatorname{id}_{\alpha} \theta_{0}+\beta \theta_{0} \theta_{1}, \\
m_{01}= & \operatorname{id}_{e}+\alpha\left(\theta_{0}-\theta_{1}\right)+[\alpha \otimes \alpha+\mathrm{s}(\beta)] \theta_{0} \theta_{1}, \\
n_{012}= & \operatorname{id}_{i_{e}}+\operatorname{id}_{\alpha}\left(\theta_{0}-\theta_{2}\right)+\beta\left(\theta_{0} \theta_{1}+\theta_{1} \theta_{2}-\theta_{0} \theta_{2}\right)+ \\
& \quad+\operatorname{id}_{\alpha \otimes \alpha+s(\beta)} \theta_{0} \theta_{2}+\left[\operatorname{id}_{\alpha} \otimes \beta-\beta \otimes \operatorname{id}_{\alpha}+\mu(\alpha, \alpha, \alpha)\right] \theta_{0} \theta_{1} \theta_{2},
\end{aligned}
$$

where $\mu(\alpha, \alpha, \alpha): \alpha \otimes(\alpha \otimes \alpha)-(\alpha \otimes \alpha) \otimes \alpha \Rightarrow 0$. 
Proof. The expansion of $m_{0}$ is a direct consequence of (4.28) while the expansion (4.30b) follows directly from the conditions $n_{00}=\mathrm{id}_{m_{0}}=\mathrm{id}_{\mathrm{id}_{e}}+\mathrm{id}_{\alpha} \theta_{0}$ and $n\left(\theta_{0}, 0\right)=\mathrm{id} m_{0} ; \mathrm{t}\left(n_{01}\right)=$ $m_{0}=\mathrm{id}_{e}+\alpha \theta_{0}$ implies $\mathrm{t}(\beta)=0$. The expansion (4.30c) follows from the normalisation $m_{00}=\mathrm{id}_{e}$ together with (4.30b) by comparing coefficients in $\mathrm{s}\left(n_{01}\right)=m_{01} \otimes m_{1}$, where we used the identity

$$
\begin{aligned}
& \left(\mathrm{id}_{e}+\alpha\left(\theta_{0}-\theta_{2}\right)+\alpha_{2} \theta_{0} \theta_{2}\right) \otimes\left(\mathrm{id}_{e}+\alpha \theta_{2}\right)= \\
& \quad=\left(\operatorname{id}_{e}+\left(\alpha-\frac{1}{2} \alpha_{2}\left(\theta_{0}+\theta_{2}\right)\right)\left(\theta_{0}-\theta_{2}\right)\right) \otimes\left(\mathrm{id}_{e}+\alpha \theta_{2}\right) \\
& \quad=\operatorname{id}_{e}+\alpha \theta_{0}+\left(\alpha_{2}-\alpha \otimes \alpha\right) \theta_{0} \theta_{2}
\end{aligned}
$$

to evaluate the product.

To derive the expansion (4.30d), we use $n\left(\theta_{0}, \theta_{1}, 0\right)=n\left(\theta_{0}, \theta_{1}\right)$ together with the normalisation $n_{001}=\mathrm{id}_{m_{01}}$ and $n_{011}=\mathrm{id}_{m_{01}}$. Hence, $n_{012}$ must be of the form

$$
n_{012}=\mathrm{id}_{\mathrm{id}_{e}}+\mathrm{id}_{\alpha}\left(\theta_{0}-\theta_{2}\right)+\beta\left(\theta_{0} \theta_{1}+\theta_{1} \theta_{2}-\theta_{0} \theta_{2}\right)+\mathrm{id}_{\alpha \otimes \alpha+\mathrm{s}(\beta)} \theta_{0} \theta_{2}+\gamma \theta_{0} \theta_{1} \theta_{2} .
$$

for some 2-cell $\gamma \in \mathfrak{n}[-3]$. To find $\gamma$ from (4.27) and (4.30a)-(4.30c), we require an expansion of the associator $\mathrm{a}_{m_{01}, m_{12}, m_{2}}$. Since according to Proposition $2.28 \mathrm{a}_{\mathrm{id}_{e}, m, m^{\prime}}, \mathrm{a}_{m, \mathrm{id}_{e}, m^{\prime}}$, and $\mathrm{a}_{m, m^{\prime}}$,ide $\mathrm{i}_{e}$ are trivial for all $m, m^{\prime} \in M$, we can write

$$
\mathrm{a}_{m_{01}, m_{12}, m_{2}}=\mathrm{id}_{m_{01} \otimes\left(m_{12} \otimes m_{2}\right)}+\mu(\alpha, \alpha, \alpha) \theta_{0} \theta_{1} \theta_{2},
$$

defining a linearised 2-cell $\mu(\alpha, \alpha, \alpha): \alpha \otimes(\alpha \otimes \alpha)-(\alpha \otimes \alpha) \otimes \alpha \Rightarrow 0$. In order to evaluate (4.27) for coboundaries given in (4.30), we note that (4.32) can be rewritten as

$$
n_{012}=\mathrm{id}_{\mathrm{id}_{e}}+\left[\mathrm{id}_{\alpha}+\frac{1}{2}\left(\beta-\mathrm{id}_{\alpha \otimes \alpha+\mathrm{s}(\beta)}+\gamma \theta_{1}\right)\left(\theta_{0}+\theta_{2}\right)-\beta \theta_{1}\right]\left(\theta_{0}-\theta_{2}\right)
$$

and likewise for $n_{01}=\mathrm{id}_{\mathrm{id}_{e}}+\left(\mathrm{id}_{\alpha}-\beta \theta_{1}\right) \theta_{0}$ and all the other terms appearing in (4.27). Thus, our definitions of the induced concatenation and products $\otimes$ to linear order are sufficient to evaluate (4.27). For example, we compute

$$
n_{012} \otimes \mathrm{id}_{m_{2}}=\mathrm{id}_{\mathrm{id}_{e}}+\mathrm{id}_{\alpha} \theta_{0}+\beta\left(\theta_{0} \theta_{1}+\theta_{1} \theta_{2}-\theta_{0} \theta_{2}\right)+\mathrm{id}_{\mathbf{s}(\beta)} \theta_{0} \theta_{2}+\left(\gamma+\beta \otimes \mathrm{id}_{\alpha}\right) \theta_{0} \theta_{1} \theta_{2} .
$$

Comparing the coefficient of $\theta_{0} \theta_{1} \theta_{2}$ of both sides of equation (4.27), we obtain

$$
\gamma=\operatorname{id}_{\alpha} \otimes \beta-\beta \otimes \operatorname{id}_{\alpha}+\mu(\alpha, \alpha, \alpha) \text {. }
$$

In deriving the latter, we have used $\beta \circ\left(\operatorname{id}_{\mathbf{s}(\beta)}-\beta\right)=0$, which follows immediately from Proposition 2.29.

Corollary 4.23. The induced differentials $\mathrm{d}_{\mathrm{K}}$ of $\alpha \in \mathfrak{m}[-1]$ and $\beta \in \mathfrak{n}[-2]$ with $\mathrm{t}(\beta)=0$ are given by

$$
\begin{aligned}
& \mathrm{d}_{\mathrm{K}} \alpha=-\alpha \otimes \alpha-\mathrm{s}(\beta), \\
& \mathrm{d}_{\mathrm{K}} \beta=-\mathrm{id}_{\alpha} \otimes \beta+\beta \otimes \mathrm{id}_{\alpha}-\mu(\alpha, \alpha, \alpha) .
\end{aligned}
$$


Proof. This is a direct consequence of the application of the differential $\mathrm{d}_{\mathrm{K}}$ to $\left\{n_{012}\right\}$ as given in Proposition 4.22. Alternatively, the first of these equations can also be obtained from the application of $\mathrm{d}_{\mathrm{K}}$ to $\left\{m_{01}\right\}$.

From equations (4.37), we can now extract the Chevalley-Eilenberg algebra of a 2term $L_{\infty}$-algebra. In particular, let $\left(\tau_{i}\right)$ and $\left(\sigma_{m}\right)$ be bases of $\mathfrak{w}:=\mathfrak{m}=T_{\operatorname{id}_{e}} M$ and $\mathfrak{v}:=\operatorname{ker}(\mathrm{t}) \subseteq \mathfrak{n}=T_{\operatorname{id}_{\mathrm{id}_{e}}} N$, respectively, and let $\left(\check{\tau}^{i}\right)$ and $\left(\check{\sigma}^{m}\right)$ be the corresponding dual bases of $\mathfrak{w}^{\vee}$ and $\mathfrak{v}^{\vee}$. The equations (4.37) should be regarded as the evaluation of

$$
\begin{aligned}
\mathrm{d}_{\mathrm{CE}} \check{\tau}^{i} & =-s_{m}^{i} \check{\sigma}^{m}-\frac{1}{2} f_{j k}^{i} \check{\tau}^{j} \wedge \check{\tau}^{k}, \\
\mathrm{~d}_{\mathrm{CE}} \check{\sigma}^{m} & =-\frac{1}{2} c_{\mathrm{in}}^{m}\left(\check{\tau}^{i} \wedge \check{\sigma}^{n}-\check{\sigma}^{n} \wedge \check{\tau}^{i}\right)+\frac{1}{3 !} d_{i j k}^{m} \check{\tau}^{i} \wedge \check{\tau}^{j} \wedge \check{\tau}^{k},
\end{aligned}
$$

at $\check{\tau}^{i}=\alpha^{i}$ and $\check{\sigma}^{m}=\beta^{m}$ with $\alpha=\alpha^{i} \tau_{j}$ and $\beta=\beta^{m} \sigma_{m}$. The constants $s_{m}^{i}, f_{j k}^{i}, c_{\mathrm{in}}^{m}$, and $d_{i j k}^{m}$ are the generalised structure constants of the 2 -term $L_{\infty}$-algebra $\mathfrak{v} \stackrel{\mu_{1}}{\longrightarrow} \mathfrak{w}$ :

$$
\begin{aligned}
\mu_{1}\left(\sigma_{m}\right) & =-s_{m}^{i} \tau_{i}, \\
\mu_{2}\left(\tau_{i}, \tau_{j}\right) & =f_{i j}^{k} \tau_{k} \quad \text { and } \quad \mu_{2}\left(\tau_{i}, \sigma_{m}\right)=c_{i m}^{n} \sigma_{n}, \\
\mu_{3}\left(\tau_{i}, \tau_{j}, \tau_{k}\right) & =-d_{i j k}^{m} \sigma_{m} .
\end{aligned}
$$

The additional signs are included to match our overall conventions, cf. Remark 2.40. The higher homotopy Jacobi identities follow from the fact that $\mathrm{d}_{\mathrm{CE}}^{2}=\mathrm{d}_{\mathrm{K}}^{2}=0$ [41].

We sum up our findings in the following theorem.

Theorem 4.24. For a semistrict Lie 2-group $\mathscr{G}=(M, N)$, the functor from the category of smooth manifolds $X$ to the category of $\mathscr{G}$-valued descent data on surjective submersions $\mathbb{R}^{1 \mid 0} \times X \rightarrow X$ is parameterised by elements of $\mathfrak{w}[-1] \oplus \mathfrak{v}[-2]$, where $\mathfrak{v} \rightarrow \mathfrak{w}$ is the 2-term $L_{\infty}$-algebra for which $\mathfrak{w}:=T_{\operatorname{id}_{e}} M$ and $\mathfrak{v}:=\operatorname{ker}(\mathrm{t}) \subseteq T_{\mathrm{id}_{\mathrm{id}_{e}}} N$. The action of the differential $\mathrm{d}_{\mathrm{K}}$ on the descent data yields the Chevalley-Eilenberg differential of the 2-term $L_{\infty}$-algebra $\mathfrak{v} \rightarrow \mathfrak{w}$.

Analogously to Lie groups, we would like to consider an equivalent descent datum and compare the change of the resulting Chevalley-Eilenberg algebra. This will eventually give us equivalent an parameterisation $(\tilde{\alpha}, \tilde{\beta}) \in \mathfrak{w}[-1] \oplus \mathfrak{v}[-2]$ obtained from $(\alpha, \beta) \in$ $\mathfrak{w}[-1] \oplus \mathfrak{v}[-2]$.

Lemma 4.25. Equivalent descent data $\left(\left\{\tilde{m}_{01}\right\},\left\{\tilde{n}_{012}\right\}\right)$ and $\left(\left\{m_{01}\right\},\left\{n_{012}\right\}\right)$ are related by a degree-2 Čech coboundary $\left(\left\{p_{0}:=p\left(\theta_{0}\right)\right\},\left\{q_{01}:=q\left(\theta_{0}, \theta_{1}\right)\right\}\right)$ according to

$$
\begin{aligned}
q_{01}: \tilde{m}_{01} \otimes p_{1} \Rightarrow & p_{0} \otimes m_{01}, \\
q_{02} \circ\left(\tilde{n}_{012} \otimes \operatorname{id}_{p_{2}}\right)= & \left(\operatorname{id}_{p_{0}} \otimes n_{012}\right) \circ \mathrm{a}_{p_{0}, m_{01}, m_{12}} \circ\left(q_{01} \otimes \operatorname{id}_{m_{12}}\right) \circ \\
& \circ \mathrm{a}_{\tilde{m}_{01}, p_{1}, m_{12}}^{-1} \circ\left(\operatorname{id}_{\tilde{m}_{01}} \otimes q_{12}\right) \circ \mathrm{a}_{\tilde{m}_{01}, \tilde{m}_{12}, p_{2}}
\end{aligned}
$$

with

$$
p_{0}=p-\mathrm{d}_{\mathrm{K}} p \theta_{0} \quad \text { and } \quad q_{01}=\mathrm{id}_{p}+\lambda_{p}\left(\theta_{0}-\theta_{1}\right)-\mathrm{id}_{\mathrm{d}_{\mathrm{K}} p} \theta_{1}-\mathrm{d}_{\mathrm{K}} \lambda_{p} \theta_{0} \theta_{1}
$$

for some $p \in N$ and $\lambda_{p} \in T_{p}[-1] N$. 
Proof. The expansion for $q_{01}$ in (4.41) follows from $q_{00}=\mathrm{id}_{p_{0}}$, cf. Remark 3.27, together with $\mathrm{d}_{\mathrm{K}} \operatorname{id}_{\mathrm{d}_{\mathrm{K}} p}=0$.

Note that contrary to the previously considered coboundaries, $p_{0}$ and $q_{01}$ are points in $M$ near $p$ and in $N$ near $\operatorname{id}_{p}$, respectively. Our formulæ for linearising the structure maps at $p$ and $\operatorname{id}_{p}$, however, remain essentially the same, cf. Remark 4.18.

Following Proposition 2.14, we may now combine the coboundaries $\left(\left\{m_{0}\right\},\left\{n_{01}\right\}\right)$ and $\left(\left\{p_{0}\right\},\left\{q_{01}\right\}\right)$ appearing in (4.27) to a new coboundary $\left(\left\{m_{0}^{\prime}\right\},\left\{n_{01}^{\prime}\right\}\right)$. The diagram

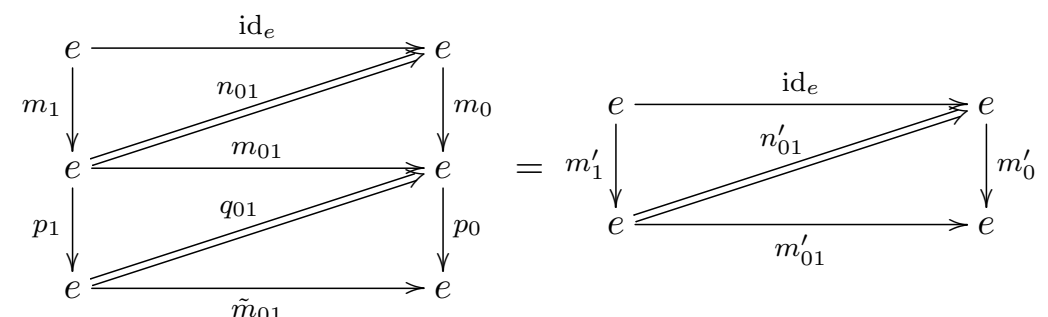

yields the formulæ

$$
\begin{aligned}
& m_{0}^{\prime}=p_{0} \otimes m_{0}, \\
& n_{01}^{\prime}: \tilde{m}_{01} \otimes m_{1}^{\prime} \Rightarrow m_{0}^{\prime}, \\
& n_{01}^{\prime}=\left(\operatorname{id}_{p_{0}} \otimes n_{01}\right) \circ \mathrm{a}_{p_{0}, m_{01}, m_{1}} \circ\left(q_{01} \otimes \mathrm{id}_{m_{1}}\right) \circ \mathrm{a}_{\tilde{m}_{01}, p_{1}, m_{1}}^{-1} .
\end{aligned}
$$

Hence, $\tilde{n}_{012}$ obeys

$$
n_{02}^{\prime} \circ\left(\tilde{n}_{012} \otimes \mathrm{id}_{m_{2}^{\prime}}\right)=n_{01}^{\prime} \circ\left(\mathrm{id}_{\tilde{m}_{01}} \otimes n_{12}^{\prime}\right) \circ \mathrm{a}_{\tilde{m}_{01}, \tilde{m}_{12}, m_{2}^{\prime}} .
$$

Comparing the parameterisation of the coboundary $\left(\left\{m_{0}\right\},\left\{n_{01}\right\}\right)$ with that of $\left(\left\{m_{0}^{\prime}\right\}\right.$, $\left.\left\{n_{01}^{\prime}\right\}\right)$ is not straightforward as their expansions in the Graßmann-odd coordinates are different. In particular $m_{0}^{\prime}$ and $n_{01}^{\prime}$ are not the same as $\tilde{m}_{0}:=\tilde{m}\left(\theta_{0}, 0\right)$ and $\tilde{n}_{01}:=\tilde{n}\left(\theta_{0}, \theta_{1}, 0\right)$, in general. To remedy this, we apply a modification isomorphism $\left\{o_{0}: m_{0}^{\prime} \Rightarrow \tilde{m}_{0} \otimes p\right\}$, taking us from the coboundary $\left(\left\{m_{0}^{\prime}\right\},\left\{n_{01}^{\prime}\right\}\right)$ to the coboundary $\left(\left\{\tilde{m}_{0}\right\},\left\{\hat{n}_{01}\right\}\right)$ :

$$
o_{0} \circ n_{01}^{\prime}=\hat{n}_{01} \circ\left(\operatorname{id}_{\tilde{m}_{01}} \otimes o_{1}\right) \quad \text { with } \quad\left\{o_{0}:=o\left(\theta_{0}\right):=q^{-1}\left(\theta_{0}, 0\right)\right\},
$$

where $\hat{n}_{01}: \tilde{m}_{01} \otimes\left(\tilde{m}_{1} \otimes p\right) \Rightarrow \tilde{m}_{0} \otimes p$. It is then easy to see that

$$
\tilde{m}(0)=\mathrm{id}_{e}, \quad \hat{n}_{00}=\mathrm{id}_{\tilde{m}_{0} \otimes p}, \quad \text { and } \quad \hat{n}\left(\theta_{0}, 0\right)=\mathrm{id}_{\tilde{m}_{0} \otimes p}
$$

and hence,

$$
\hat{n}_{02} \circ\left(\tilde{n}_{012} \otimes \operatorname{id}_{\tilde{m}_{2} \otimes p}\right)=\hat{n}_{01} \circ\left(\mathrm{id}_{\tilde{m}_{01}} \otimes \hat{n}_{12}\right) \circ \mathrm{a}_{\tilde{m}_{01}, \tilde{m}_{12}, \tilde{m}_{2} \otimes p} .
$$

For $\theta_{2}=0$, this equation implies that

$$
\tilde{n}_{01} \otimes \mathrm{id}_{p}=\hat{n}_{01} \circ \mathrm{a}_{\tilde{m}_{01}, \tilde{m}_{1}, p} .
$$

Altogether, we have thus constructed a coboundary $\left(\left\{\tilde{m}_{0}\right\},\left\{\tilde{n}_{01}\right\}\right)$ representing the equivalent descent data $\left(\left\{\tilde{m}_{01}, \tilde{n}_{012}\right\}\right) \sim\left(\left\{m_{01}, n_{012}\right\}\right)$ according to

$$
\tilde{n}_{02} \circ\left(\tilde{n}_{012} \otimes \mathrm{id}_{\tilde{m}_{2}}\right)=\tilde{n}_{01} \circ\left(\mathrm{id}_{\tilde{m}_{01}} \otimes \tilde{n}_{12}\right) \circ \mathrm{a}_{\tilde{m}_{01}, \tilde{m}_{12}, \tilde{m}_{2}} .
$$

These considerations then lead to the following theorem. 
Theorem 4.26. Let $\left(\left\{m_{01}\right\},\left\{n_{012}\right\}\right)$ be a descent datum parametrised by $(\alpha, \beta) \in \mathfrak{m}[-1] \oplus$ $\mathfrak{n}[-2]$ with $\mathrm{t}(\beta)=0$. Furthermore, let $\left(\left\{\tilde{m}_{01}\right\},\left\{\tilde{n}_{012}\right\}\right)$ be an equivalent descent datum that is parametrised by $(\tilde{\alpha}, \tilde{\beta}) \in \mathfrak{m}[-1] \oplus \mathfrak{n}[-2]$ with $\mathrm{t}(\tilde{\beta})=0$. Then $\tilde{\alpha}$ and $\tilde{\beta}$ are expressed in terms of $\alpha$ and $\beta$ according to

$$
\begin{aligned}
\lambda_{p}: \tilde{\alpha} \otimes p \Rightarrow & p \otimes \alpha-\mathrm{d}_{\mathrm{K}} p, \\
\tilde{\beta} \otimes \operatorname{id}_{p}= & \mu(\tilde{\alpha}, \tilde{\alpha}, p)+\left[\operatorname{id}_{p} \otimes \beta+\mu(p, \alpha, \alpha)\right] \circ \\
& \circ\left[-\mathrm{d}_{\mathrm{K}} \lambda_{p}-\lambda_{p} \otimes \mathrm{id}_{\alpha}-\mu(\tilde{\alpha}, p, \alpha)\right] \circ \\
& \circ\left[-\mathrm{id}_{\mathbf{s}\left(\mathrm{d}_{\mathrm{K}} \lambda_{p}\right)}-\mathrm{id}_{\tilde{\alpha}} \otimes\left(\lambda_{p}+\mathrm{id}_{\mathrm{d}_{\mathrm{K}} p}\right)\right],
\end{aligned}
$$

where $p \in M$ and $\lambda_{p} \in T_{p}[-1] N$. By construction, equations (4.37) are invariant under this equivalence relation.

Proof. We follow the arguments around (4.40)-(4.49) so that the expansions of $\left\{m_{0}\right\},\left\{n_{01}\right\}$, $\left\{m_{01}\right\}$, and $\left\{n_{012}\right\}$ and $\left\{\tilde{m}_{0}\right\},\left\{\tilde{n}_{01}\right\},\left\{\tilde{m}_{01}\right\}$, and $\left\{\tilde{n}_{012}\right\}$, are those given in Proposition 4.22, with tilded coefficients for tilded quantities. The expansion of the coboundary $\left(\left\{p_{0}\right\},\left\{q_{01}\right\}\right)$ are given in Lemma 4.25 .

Since $q_{01}: \tilde{m}_{01} \otimes p_{1} \Rightarrow p_{0} \otimes m_{01}$, we find by computing the source and target and using the expansions (see also Proposition 4.22 and Corollary 4.23)

$$
\begin{aligned}
& m_{01}=\mathrm{id}_{e}+\alpha\left(\theta_{0}-\theta_{1}\right)+[\alpha \otimes \alpha+\mathrm{s}(\beta)] \theta_{0} \theta_{1}=\mathrm{id}_{e}+\alpha\left(\theta_{0}-\theta_{1}\right)-\mathrm{d}_{\mathrm{K}} \alpha \theta_{0} \theta_{1}, \\
& \tilde{m}_{01}=\mathrm{id}_{e}+\tilde{\alpha}\left(\theta_{0}-\theta_{1}\right)+[\tilde{\alpha} \otimes \tilde{\alpha}+\mathrm{s}(\tilde{\beta})] \theta_{0} \theta_{1}=\operatorname{id}_{e}+\tilde{\alpha}\left(\theta_{0}-\theta_{1}\right)-\mathrm{d}_{\mathrm{K}} \tilde{\alpha} \theta_{0} \theta_{1},
\end{aligned}
$$

that

$$
\begin{aligned}
\lambda_{p}: \tilde{\alpha} \otimes p & \Rightarrow p \otimes \alpha-\mathrm{d}_{\mathrm{K}} p, \\
\mathrm{~d}_{\mathrm{K}} \lambda_{p}:-\mathrm{d}_{\mathrm{K}} \tilde{\alpha} \otimes p+\tilde{\alpha} \otimes \mathrm{d}_{\mathrm{K}} p & \Rightarrow-\mathrm{d}_{\mathrm{K}} p \otimes \alpha-p \otimes \mathrm{d}_{\mathrm{K}} \alpha,
\end{aligned}
$$

thus verifying (4.50a).

To compute $n_{01}^{\prime}$ from (4.43), we need to establish the explicit form of the two associators $\mathrm{a}_{p_{0}, m_{01}, m_{1}}$ and $\mathrm{a}_{\tilde{m}_{01}, p_{1}, m_{1}}^{-1}$. Both of these become trivial for $\theta_{0}=\theta_{1}$ or $\theta_{1}=0$. We therefore have the following expansions,

$$
\begin{aligned}
& \mathrm{a}_{p_{0}, m_{01}, m_{1}}=: \operatorname{id}_{p_{0} \otimes\left(m_{01} \otimes m_{1}\right)}+\mu(p, \alpha, \alpha) \theta_{0} \theta_{1}, \\
& \mathrm{a}_{\tilde{m}_{01}, p_{1}, m_{1}}^{-1}=: \operatorname{id}_{\left(\tilde{m}_{01} \otimes p_{1}\right) \otimes m_{1}}-\mu(\tilde{\alpha}, p, \alpha) \theta_{0} \theta_{1},
\end{aligned}
$$

defining two maps, which we both denote by $\mu$ :

$$
\begin{aligned}
& \mu(p, \alpha, \alpha): p \otimes(\alpha \otimes \alpha)-(p \otimes \alpha) \otimes \alpha \Rightarrow 0, \\
& \mu(\tilde{\alpha}, p, \alpha): \tilde{\alpha} \otimes(p \otimes \alpha)-(\tilde{\alpha} \otimes p) \otimes \alpha \Rightarrow 0 .
\end{aligned}
$$

Upon substituting these expressions together with those for $\left\{p_{01}\right\},\left\{q_{0}\right\}$ and $\left\{n_{01}\right\}$, $\left\{m_{1}\right\}$ into (4.43), we find

$$
\begin{aligned}
n_{01}^{\prime}= & \operatorname{id}_{p}+\left(\theta_{0}-\theta_{1}\right) \lambda_{p}+\operatorname{id}_{p \otimes \alpha-\mathrm{d}_{\mathrm{K}} p} \theta_{1}+ \\
& +\left[\operatorname{id}_{p} \otimes \beta+\mu(p, \alpha, \alpha)\right] \circ\left[-\mathrm{d}_{\mathrm{K}} \lambda_{p}-\lambda_{p} \otimes \mathrm{id}_{\alpha}-\mu(\tilde{\alpha}, p, \alpha)\right] \theta_{0} \theta_{1} .
\end{aligned}
$$


Here, we relied on the fact that each of the terms in (4.43) can be written as $\operatorname{id}_{p}+\theta_{0} \pi_{1}+\theta_{1} \pi_{2}$, where $\pi_{1,2} \in T_{p}[-1] N$, and for these, the linearised concatenation is well-defined.

Finally, we perform the modification transformation $o_{0}: m_{0}^{\prime} \Rightarrow \tilde{m}_{0} \otimes p$ with $\left\{o_{0}^{-1}=\right.$ $\left.q\left(\theta_{0}, 0\right)\right\}$ which we have introduced in (4.45),

$$
o_{0} \circ n_{01}^{\prime}=\hat{n}_{01} \circ\left(\operatorname{id}_{\tilde{m}_{01}} \otimes o_{1}\right) \Longleftrightarrow o_{0}^{-1} \circ \hat{n}_{01}=n_{01}^{\prime} \circ\left(\operatorname{id}_{\tilde{m}_{01}} \otimes o_{1}^{-1}\right),
$$

to obtain $\hat{n}_{01}: \tilde{m}_{01} \otimes\left(\tilde{m}_{1} \otimes p\right) \Rightarrow \tilde{m}_{0} \otimes p$. Using (4.48) and $\left\{o_{0}^{-1}=q\left(\theta_{0}, 0\right)\right\}$, this can be rewritten as

$$
q\left(\theta_{0}, 0\right) \circ\left(\tilde{n}_{01} \otimes \mathrm{id}_{p}\right) \circ \mathrm{a}_{\tilde{m}_{01}, \tilde{m}_{1}, p}^{-1}=n_{01}^{\prime} \circ\left[\mathrm{id}_{\tilde{m}_{01}} \otimes q\left(\theta_{1}, 0\right)\right] .
$$

To evaluate this expression we need to fix the expansion of the associator, which we do according to

$$
\mathrm{a}_{\tilde{m}_{01}, \tilde{m}_{1}, p}^{-1}=: \operatorname{id}_{\left(\tilde{m}_{01} \otimes \tilde{m}_{1}\right) \otimes p}-\mu(\tilde{\alpha}, \tilde{\alpha}, p) \theta_{0} \theta_{1},
$$

where $\mu(\tilde{\alpha}, \tilde{\alpha}, p): \tilde{\alpha} \otimes(\tilde{\alpha} \otimes p)-(\tilde{\alpha} \otimes \tilde{\alpha}) \otimes p \Rightarrow 0$. Substituting this expression, (4.41), and (4.55) into (4.57), we find after some algebraic manipulations that $\tilde{n}_{01}=\mathrm{id}_{e}+\operatorname{id}_{\tilde{\alpha}} \theta_{0}+$ $\tilde{\beta} \theta_{0} \theta_{1}$ with

$$
\begin{aligned}
\tilde{\beta} \otimes \mathrm{id}_{p}= & \mu(\tilde{\alpha}, \tilde{\alpha}, p)+\left[\operatorname{id}_{p} \otimes \beta+\mu(p, \alpha, \alpha)\right] \circ \\
& \circ\left[-\mathrm{d}_{\mathrm{K}} \lambda_{p}-\lambda_{p} \otimes \mathrm{id}_{\alpha}-\mu(\tilde{\alpha}, p, \alpha)\right] \circ\left[-\mathrm{id}_{\mathbf{s}\left(\mathrm{d}_{\mathrm{K}} \lambda_{p}\right)}-\mathrm{id}_{\tilde{\alpha}} \otimes\left(\lambda_{p}+\mathrm{id}_{\mathrm{d}_{\mathrm{K}} p}\right)\right],
\end{aligned}
$$

verifying (4.50b). Note that $\mathrm{t}(\tilde{\beta})=0$ as required. This concludes the proof.

Finally, we would like to emphasise that given $\lambda_{p} \in T_{p}[-1] N$, we can always construct a $\lambda \in \mathfrak{n}[-1]$ and vice versa.

Definition 4.27. Let $p \in M$ and $\lambda_{p} \in T_{p}[-1] N$ be given as in Theorem 4.26. We define a 2-cell $\lambda \in \mathfrak{n}[-1]$ by setting

$$
\lambda:=\left(\lambda_{p} \otimes \operatorname{id}_{\bar{p}}\right) \circ \mathrm{a}_{\tilde{\alpha}, p, \bar{p}}^{-1},
$$

that is, $\lambda: \tilde{\alpha} \Rightarrow(p \otimes \alpha) \otimes \bar{p}-\mathrm{d}_{\mathrm{K}} p \otimes \bar{p}$, where $\bar{p} \in M$ with $p \otimes \bar{p}=\mathrm{id}_{e}=\bar{p} \otimes p$ and $\mathrm{a}_{\tilde{\alpha}, p, \bar{p}}:(\tilde{\alpha} \otimes p) \otimes \tilde{p} \Rightarrow \tilde{\alpha} \otimes(p \otimes \tilde{p})$. In addition, we define a 2-cell $\lambda_{0} \in \mathfrak{v}[-1]$ by setting

$$
\lambda_{0}:=\lambda-\operatorname{id}_{(p \otimes \alpha) \otimes \bar{p}-\mathrm{d}_{K} p \otimes \bar{p}},
$$

that is, $\lambda_{0}: \tilde{\alpha}-(p \otimes \alpha) \otimes \bar{p}+\mathrm{d}_{\mathrm{K}} p \otimes \bar{p} \Rightarrow 0$ with an intuitive notation to be understood.

Proposition 4.28. Given $\lambda$ as in Definition 4.27, we have

$$
\lambda_{p}=\mathrm{a}_{p \otimes \alpha+\mathrm{d}_{\mathrm{K}} p, \bar{p}, p} \circ\left[\left(\lambda \circ \mathrm{a}_{\tilde{\alpha}, p, \bar{p}}\right) \otimes \mathrm{id}_{p}\right] \circ \mathrm{a}_{\tilde{\alpha} \otimes p, \bar{p}, p}^{-1} .
$$

Proof. Due to the naturalness of the associator, it is straightforward to see that $\lambda_{p}$ can be expressed in terms of $\lambda$ in the above way. 


\subsection{Example: strict Lie 2-groups}

As a consistency check, let us now consider a class of examples. Since it is notoriously difficult to construct non-trivial examples of Lie 2-groups which are not strict, we have to consider the strict case. That is, we start from descent data for strict principal 2-bundles in the general Lie 2-group framework. For such bundles, we have $n_{012}=\ell_{02}^{-1} \circ\left(\ell_{01} \otimes \ell_{12}\right)$ as was discussed in Remark 3.24. One can check that then

$$
\ell_{01}=n_{01} \otimes \operatorname{id}_{\bar{m}_{1}}=\operatorname{id}_{i_{e}}+\operatorname{id}_{\alpha}\left(\theta_{0}-\theta_{1}\right)+\left(\beta+\operatorname{id}_{\alpha \otimes \alpha}\right) \theta_{0} \theta_{1},
$$

which yields the following.

Lemma 4.29. For strict Lie 2-groups, the functor between the category of smooth manifolds $X$ and the category of $\mathscr{G}$-valued descent data on $\mathbb{R}^{0 \mid 1} \times X \rightarrow X$ reads as

$$
\begin{aligned}
m_{01}= & \operatorname{id}_{e}+\alpha\left(\theta_{0}-\theta_{1}\right)+\theta_{0} \theta_{1}[\alpha \otimes \alpha+\mathrm{s}(\beta)], \\
n_{012}= & \operatorname{id}_{\mathrm{id}_{e}}+\operatorname{id}_{\alpha}\left(\theta_{0}-\theta_{2}\right)+\beta\left(\theta_{0} \theta_{1}+\theta_{1} \theta_{2}-\theta_{0} \theta_{2}\right)+ \\
& +\operatorname{id}_{\alpha \otimes \alpha+\mathrm{s}(\beta)} \theta_{0} \theta_{2}+\left(\operatorname{id}_{\alpha} \otimes \beta-\beta \otimes \mathrm{id}_{\alpha}\right) \theta_{0} \theta_{1} \theta_{2},
\end{aligned}
$$

which implies

$$
\mathrm{d}_{\mathrm{K}} \alpha=-\alpha \otimes \alpha-\mathrm{s}(\beta) \quad \text { and } \quad \mathrm{d}_{\mathrm{K}} \beta=-\mathrm{id}_{\alpha} \otimes \beta+\beta \otimes \mathrm{id}_{\alpha} .
$$

To compare with the literature, we need to translate these results into expressions using crossed modules of Lie groups.

Proposition 4.30. In terms of crossed modules of Lie groups $(\mathrm{H} \stackrel{\rightarrow}{\rightarrow} \mathrm{G}, \triangleright)$, the functor between the category of smooth manifolds $X$ and the category of $(\mathrm{H} \stackrel{\partial}{\rightarrow} \mathrm{G}, \triangleright)$-valued descent data on $\mathbb{R}^{0 \mid 1} \times X \rightarrow X$ is given by Cech 1- and 2-cochains $\left\{g_{01}\right\}$ and $\left\{h_{012}\right\}$ with values in the Lie groups $\mathrm{G}$ and $\mathrm{H}$, respectively. These are parameterised by $a \in \mathfrak{g}[-1]$ and $b \in \mathfrak{h}[-2]$, where $\mathfrak{g}$ and $\mathfrak{h}$ are the Lie algebras of $\mathrm{G}$ and $\mathrm{H}$, according to

$$
g_{01}=\mathbb{1}_{\mathrm{G}}+a\left(\theta_{0}-\theta_{1}\right)+\left\{\frac{1}{2}[a, a]-\partial(b)\right\} \theta_{0} \theta_{1}
$$

and

$$
h_{012}=\mathbb{1}_{\mathbf{H}}+b\left(\theta_{0} \theta_{1}+\theta_{1} \theta_{2}-\theta_{0} \theta_{2}\right)+(a \triangleright b) \theta_{0} \theta_{1} \theta_{2} .
$$

The action of the differential $\mathrm{d}_{\mathrm{K}}$ translates to

$$
\mathrm{d}_{\mathrm{K}} a=-\frac{1}{2}[a, a]+\partial(b) \quad \text { and } \quad \mathrm{d}_{\mathrm{K}} b=-a \triangleright b .
$$

Proof. Starting from (4.64) and (4.65), we follow Proposition 2.36 and define $G:=M$ and $\mathrm{H}=\operatorname{ker}(\mathrm{t}) \subseteq N$. The products on $\mathrm{G}$ and $\mathrm{H}$, the action $\triangleright$ and the map $\partial$ are defined according to equation (2.20). We then identify

$$
g_{01}=m_{01} \quad \text { and } \quad h_{012}=n_{012} \otimes \mathrm{id}_{\bar{m}_{02}},
$$


which implies $\alpha=a \in \mathfrak{g}[-1]$ and $\beta=b$. Clearly, this identification is reversible and therefore an equivalence. The cocycle relations (4.25b) for $\left(\left\{m_{01}\right\},\left\{n_{012}\right\}\right)$ are then equivalent to those for $\left(\left\{g_{01}\right\},\left\{h_{012}\right\}\right)$, cf. (3.28a), using the identifications under Proposition 2.36. In the strict case, $\alpha$ and $\beta$ take values in a 2-term $L_{\infty}$-algebra with trivial associator, which forms a differential crossed module. From the actions of $d_{K}$ given in (4.65) as well as equations (4.38) and (4.39), we read off that the tensor products $\alpha \otimes \alpha$ and $\operatorname{id}_{\alpha} \otimes \beta-\beta \otimes \mathrm{id}_{\alpha}$ turn into the commutator and the action of $\mathrm{G}$ onto $\mathrm{H}$.

These are the expressions that were already obtained in Jurčo [39].

Furthermore, combining the results of Theorem 4.26 and Definition 4.27 with the interchange law (2.5), we arrive after a few algebraic manipulations at

$$
\begin{aligned}
\lambda: \tilde{\alpha} & \Rightarrow p \otimes \alpha \otimes \bar{p}-\mathrm{d}_{\mathrm{K}} p \otimes \bar{p}, \\
\tilde{\beta} & =\left[\operatorname{id}_{p} \otimes \beta \otimes \operatorname{id}_{\bar{p}}\right] \circ\left[-\mathrm{d}_{\mathrm{K}} \lambda-\lambda \otimes \lambda\right] .
\end{aligned}
$$

Translated into crossed modules of Lie groups, this takes the following form.

Proposition 4.31. Let $\left(\left\{g_{01}\right\},\left\{h_{012}\right\}\right)$ be a descent datum that is parameterised by $a \in$ $\mathfrak{g}[-1]$ and $b \in \mathfrak{h}[-2]$. Furthermore, let $\left(\left\{\tilde{g}_{01}\right\},\left\{\tilde{h}_{012}\right\}\right)$ be an equivalent descent datum that is parameterised by $\tilde{a} \in \mathfrak{g}[-1]$ and $\tilde{b} \in \mathfrak{h}[-2]$. Then, $(a, b)$ and $(\tilde{a}, \tilde{b})$ are related by the following equations:

$$
\begin{aligned}
& \tilde{a}=p a p^{-1}+p \mathrm{~d}_{\mathrm{K}} p^{-1}-\partial\left(\lambda^{\mathfrak{h}}\right), \\
& \tilde{b}=p \triangleright b-\mathrm{d}_{\mathrm{K}} \lambda^{\mathfrak{h}}-\tilde{a} \triangleright \lambda^{\mathfrak{h}}-\frac{1}{2}\left[\lambda^{\mathfrak{h}}, \lambda^{\mathfrak{h}}\right]
\end{aligned}
$$

for $p \in G$ and $\lambda^{\mathfrak{h}} \in \mathfrak{h}[-1]$.

Proof. We again follow Proposition 2.36, which justifies the appearance of $p$ in (4.70) after identifying

$$
\tilde{a}=\tilde{\alpha}, \quad \tilde{b}=\tilde{\beta}, \quad \text { and } \quad \lambda^{\mathfrak{h}}=\lambda-\mathrm{id}_{p \otimes \alpha \otimes \bar{p}-\mathrm{d}_{\mathrm{K}} p \otimes \bar{p}} .
$$

More specifically, (4.70a) immediately follows from computing $\mathrm{s}\left(\lambda^{\mathfrak{h}}\right)=-\partial\left(\lambda^{\mathfrak{h}}\right)$. Recall that $\operatorname{id}_{p} \otimes \beta \otimes \operatorname{id}_{\bar{p}}$ translates to $p \triangleright b$. Using Proposition 4.17 together with the identity $\mathrm{s}(\beta)=-\mathrm{d}_{\mathrm{K}} \alpha-\alpha \otimes \alpha$, we can derive (4.70b) by a lengthy but straightforward computation from the second equation in (4.69).

\subsection{Comment on differentiation and categorical equivalence}

Recall from Proposition 2.25 that every weak 2-group is categorically equivalent to a special weak 2-group given in terms of a group $\mathrm{G}$, an Abelian group $\mathrm{H}$, a representation $\varrho$ of $\mathrm{G}$ on $\mathrm{H}$, and an element $[\mathrm{a}] \in H^{3}(\mathrm{G}, \mathrm{H})$. The corresponding Proposition 2.43 for Lie 2-algebras states that semistrict Lie 2-algebras are categorically equivalent to special Lie 2-algebras given in terms of a Lie algebra $\mathfrak{g}$, a representation $\varrho$ of $\mathfrak{g}$ on a vector space $\mathfrak{v}$, and an element $[\mathrm{J}] \in H^{3}(\mathfrak{g}, \mathfrak{v})$.

It is now tempting to assume that the natural integration process factors through categorical equivalence and therefore special Lie 2-algebras can be integrated to special Lie 
2-groups. However, Baez \& Lauda proved a no-go theorem ([4], section 8.5), which shows that certain special Lie 2-algebras can be integrated to 2-groups, which, however, can be turned into topological 2-groups only for the strict case $\mathrm{a}=0$. In particular, consider the case of a special Lie 2-algebra with $\mathfrak{v}=\mathfrak{u}(1)$. We have $H^{3}(\mathfrak{g}, \mathfrak{u}(1)) \cong \mathbb{R}$. The latter contains a lattice $\cong \mathbb{Z}$, which can be embedded into $H^{3}(\mathrm{G}, \mathrm{U}(1))$, yielding the integration to a 2-group. In the topological case, however, we have to use continuous cohomology, for which $H_{\text {cont. }}^{3}(\mathrm{G}, \mathrm{U}(1))=0$.

The differentiation of Lie 2-groups we performed in this section is the inverse operation to this integration. As integration does not commute with categorical equivalence, neither will differentiation.

\section{Semistrict higher gauge theory}

We now put the results of the previous section together and develop a description of semistrict principal 2-bundles with connective structure. We first discuss the local case, ${ }^{14}$ which can be readily derived from the Maurer-Cartan equation of an $L_{\infty}$-algebra. We then give the global description in terms of non-Abelian Deligne cohomology sets.

As before, let $X$ be a smooth manifold with covering $\mathfrak{U}=\left\{U_{a}\right\}$ and let $U \subseteq X$ be an open subset of $X$. Furthermore, let $\Omega_{X}^{p}$ be the sheaf of smooth differential $p$-forms on $X$ and set $\Omega_{X}^{\bullet}=\bigoplus_{p \geq 0} \Omega_{X}^{p}$.

\subsection{Local semistrict higher gauge theory}

Local semistrict higher gauge theory corresponds to the Maurer-Cartan equation (A.7) for a degree-1 element of the $L_{\infty}$-algebra arising from the tensor product of $\Omega_{X}^{\bullet}$ and a gauge $L_{\infty}$-algebra $L$. The corresponding infinitesimal gauge transformations are the gauge transformations of the Maurer-Cartan equation (A.8). To make this explicit, we wish to recall the following proposition.

Proposition 5.1. A tensor product of a differential graded algebra $\mathfrak{a}$ and an $L_{\infty}$-algebra $L$ comes with a natural $L_{\infty}$-structure. The grading of an element of $\mathfrak{a} \otimes L$ is the sum of its individual gradings. Moreover, for a tuple of elements $\left(a_{1} \otimes \ell_{1}, \ldots, a_{i} \otimes \ell_{i}\right)$ of $\mathfrak{a} \otimes L$, the higher products $\tilde{\mu}_{i}$ read as

$$
\tilde{\mu}_{i}\left(a_{1} \otimes \ell_{1}, \ldots, a_{i} \otimes \ell_{i}\right)= \begin{cases}\left(\mathrm{d} a_{1}\right) \otimes \ell_{1}+(-1)^{\operatorname{deg}\left(a_{1}\right)} a_{1} \otimes \mu_{1}\left(\ell_{1}\right) & \text { for } \quad i=1, \\ \chi\left(a_{1} a_{2} \cdots a_{i} \otimes \mu_{i}\left(\ell_{1}, \ldots, \ell_{i}\right)\right) & \text { for } i>1 .\end{cases}
$$

Here, the $\mu_{i}$ are the higher products in $L$, deg denotes the degrees in $\mathfrak{a}$, and $\chi= \pm 1$ is the so-called Koszul sign arising from moving graded elements of $\mathfrak{a}$ past graded elements of $L$.

Proof. The higher homotopy Jacobi identities, displayed in the appendix in (A.2), for the higher products $\tilde{\mu}_{i}$ are readily checked.

\footnotetext{
${ }^{14}$ For more details on the local case, see Sati, Schreiber \& Stasheff [42].
} 
Example 5.2. As an example, let us work out the details for the case where $\mathfrak{a}$ is the de Rham complex on $X$ and $L$ is a 2-term $L_{\infty}$-algebra. Let $U \subseteq X$ be an open subset. The tensor product of $H^{0}\left(U, \Omega_{X}^{\bullet}\right)$ and the 2-term $L_{\infty}$-algebra $\mathfrak{v} \stackrel{\mu_{1}}{\longrightarrow} \mathfrak{w}$ consists of the following graded subspaces

$$
H^{0}\left(U, \Omega_{X}^{\bullet}\right) \otimes\left(\mathfrak{v} \stackrel{\mu_{1}}{\longrightarrow} \mathfrak{w}\right) \cong \underbrace{H^{0}\left(U, \Omega_{X}^{0} \otimes \mathfrak{v}\right)}_{\text {degree }-1} \oplus \bigoplus_{p \geq 0} \underbrace{H^{0}\left(U, \Omega_{X}^{p} \otimes \mathfrak{w} \oplus \Omega_{X}^{p+1} \otimes \mathfrak{v}\right)}_{\text {degree } p} .
$$

For $\phi \in H^{0}\left(U, \Omega_{X}^{1} \otimes \mathfrak{w} \oplus \Omega_{X}^{2} \otimes \mathfrak{v}\right)$, the homotopy Maurer-Cartan equation (A.7) reads as

$$
-\tilde{\mu}_{1}(\phi)-\frac{1}{2} \tilde{\mu}_{2}(\phi, \phi)+\frac{1}{3 !} \tilde{\mu}_{3}(\phi, \phi, \phi)=0
$$

This equation is invariant under the (infinitesimal) transformations

$$
\delta \phi=\tilde{\mu}_{1}(\gamma)-\tilde{\mu}_{2}(\gamma, \phi)-\frac{1}{2} \tilde{\mu}_{3}(\gamma, \phi, \phi)
$$

for $\gamma \in H^{0}\left(U, \Omega_{X}^{0} \otimes \mathfrak{w} \oplus \Omega_{X}^{1} \otimes \mathfrak{v}\right)$.

Proposition 5.3. The homotopy Maurer-Cartan equation (5.3) and the transformations (5.4) are equivalent to the equations

$$
\begin{aligned}
& \mathcal{F}:=\mathrm{d} A+\frac{1}{2} \mu_{2}(A, A)-\mu_{1}(B)=0, \\
& H:=\mathrm{d} B+\mu_{2}(A, B)-\frac{1}{3 !} \mu_{3}(A, A, A)=0,
\end{aligned}
$$

where $A \in H^{0}\left(U, \Omega_{X}^{1} \otimes \mathfrak{w}\right)$ and $B \in H^{0}\left(U, \Omega_{X}^{2} \otimes \mathfrak{v}\right)$ and

$$
\begin{aligned}
& \delta A=\mathrm{d} \omega+\mu_{2}(A, \omega)-\mu_{1}(\Lambda), \\
& \delta B=-\mathrm{d} \Lambda-\mu_{2}(A, \Lambda)+\mu_{2}(B, \omega)+\frac{1}{2} \mu_{3}(\omega, A, A),
\end{aligned}
$$

where $\omega \in H^{0}\left(U, \Omega_{X}^{0} \otimes \mathfrak{w}\right)$ and $\Lambda \in H^{0}\left(U, \Omega_{X}^{1} \otimes \mathfrak{v}\right)$.

Proof. This trivially follows by identifying $\phi=A-B$ and $\gamma=\omega+\Lambda$ in (5.3) and (5.4).

Let us now generalise from gauge potential 1- and 2-forms $A$ and $B$ satisfying the Maurer-Cartan equation to general kinematic data for local semistrict higher gauge theory. It makes sense to relax the equation $H=0$ : a trivial calculation shows that in this case, $H$ transforms under under gauge transformations (5.6) covariantly according to $\delta H=$ $\mu_{2}(H, \omega)$. There are a number of reasons, however, why we cannot relax $\mathcal{F}=0$. Firstly, consistency of the underlying parallel transport requires $\mathcal{F}$ to vanish, just as it did in the strict case. Secondly, the above covariant transformation law is broken for non-vanishing $\mathcal{F}$, which makes it impossible to impose a self-duality condition on $H$. Such a condition, however, is expected to arise in the $\mathcal{N}=(2,0)$ superconformal field theory in six dimensions. We therefore arrive at the following definition. 
Definition 5.4. The kinematic datum of local semistrict higher gauge theory with underlying 2-term $L_{\infty}$-algebra $\mathfrak{v} \stackrel{\mu_{1}}{\longrightarrow} \mathfrak{w}$ is given by potential 1- and 2-forms $A \in H^{0}\left(U, \Omega_{X}^{1} \otimes \mathfrak{w}\right)$ and $B \in H^{0}\left(U, \Omega_{X}^{2} \otimes \mathfrak{v}\right)$, for which the 2-form fake curvature $\mathcal{F}:=\mathrm{d} A+\frac{1}{2} \mu_{2}(A, A)-\mu_{1}(B)$ vanishes. An equivalence relation between such kinematic data is generated by the infinitesimal gauge transformations described in equations (5.6).

Remark 5.5. For trivial $\mu_{3}$, the equations (5.5) reduce to the field equations for a flat connective structure of a principal 2-bundle with strict structure 2-group and equations (5.6) describe infinitesimal gauge transformations.

Note also that there are equivalence relations between gauge transformations which have the same effect on $A$ and $B$. These are given by

$$
\delta \omega=\mu_{1}(\sigma) \quad \text { and } \quad \delta \Lambda=\mathrm{d} \sigma+\mu_{2}(A, \sigma),
$$

where $\sigma \in H^{0}\left(U, \Omega_{X}^{0} \otimes \mathfrak{v}\right)$.

Remark 5.6. Finally, we would like to stress that the kinematic data, the local flatness conditions and the infinitesimal gauge transformations for local semistrict higher gauge theory based on an $n$-term $L_{\infty}$-algebras $L$ are similarly derived by considering the tensor product of $\Omega_{X}^{\bullet}$ with $L$.

\section{$5.2 \quad$ Finite gauge transformations}

Having derived curvature and infinitesimal gauge transformation for semistrict higher gauge theory, let us now turn to the finite gauge transformations. Here, we rely on the results of section 4 , and the lift to Lie $n$-algebra valued potential and curvature forms is readily obtained.

In Proposition 4.12, we showed that the equation $\mathrm{d}_{\mathrm{K}} a+\frac{1}{2}[a, a]=0$ was invariant under $a \mapsto \tilde{a}=p a p^{-1}+p \mathrm{~d}_{\mathrm{K}} p^{-1}$. Since $\mathrm{d}_{\mathrm{K}}$ and the de Rham differential $\mathrm{d}$ have the same algebraic properties, we derived the well-known statement

Proposition 5.7. If a local connection 1-form A taking values in the Lie algebra of a Lie group $\mathrm{G}$ is flat, its curvature $F:=\mathrm{d} A+\frac{1}{2}[A, A]=0$ is invariant under the transformation

$$
A \mapsto \tilde{A}=p A p^{-1}+p \mathrm{~d} p^{-1}
$$

for any $p \in H^{0}(U, \mathrm{G})$. Such transformations are called gauge transformations.

Note also the following consequence.

Corollary 5.8. At the infinitesimal level, the transformations (5.8) amount to

$$
A \mapsto \tilde{A}=\mathrm{d} \pi+[A, \pi]
$$

where $\pi \in H^{0}\left(U, \Omega_{X}^{0} \otimes \mathfrak{g}\right)$. They match the gauge transformations in Proposition 5.3 for the 2-term $L_{\infty}$-algebra $\{0\} \rightarrow \mathfrak{g}$. 
Analogously, we treat the kinematic data of local semistrict higher gauge theory. In Theorem 4.26, we showed that the equations

$$
\mathrm{d}_{\mathrm{K}} \alpha=-\alpha \otimes \alpha-\mathrm{s}(\beta) \quad \text { and } \quad \mathrm{d}_{\mathrm{K}} \beta=-\mathrm{id}_{\alpha} \otimes \beta+\beta \otimes \mathrm{id}_{\alpha}-\mu(\alpha, \alpha, \alpha)
$$

are invariant under (4.50a) and (4.50b). Again, since $d_{K}$ and $d$ have the same algebraic properties, we have derived the following statement.

Proposition 5.9. If the curvatures $\mathcal{F}$ and $H$ of local gauge potential 1 - and 2-forms $A$ and $B$ as defined in Proposition 5.3 vanish, then they are invariant under the transformation

$$
\begin{aligned}
\Lambda_{p}: \tilde{A} \otimes p \Rightarrow & p \otimes A-\mathrm{d} p, \\
\tilde{B} \otimes \mathrm{id}_{p}= & \mu(\tilde{A}, \tilde{A}, p)+\left[\operatorname{id}_{p} \otimes B+\mu(p, A, A)\right] \circ \\
& \circ\left[-\mathrm{d} \Lambda_{p}-\Lambda_{p} \otimes \mathrm{id}_{A}-\mu(\tilde{A}, p, A)\right] \circ \\
& \circ\left[-\operatorname{id}_{\mathbf{s}\left(\mathrm{d}_{p}\right)}-\operatorname{id}_{\tilde{A}} \otimes\left(\Lambda_{p}+\mathrm{id}_{\mathrm{d} p}\right)\right],
\end{aligned}
$$

where $p \in H^{0}(U, M)$ and $d^{15} \Lambda_{p} \in H^{0}\left(U, \Omega_{X}^{1} \otimes T_{p} N\right)$. We shall refer to such transformations as gauge transformations.

As a consistency check, we can linearise these gauge transformations, obtaining the transformations (5.6):

Proposition 5.10. At the infinitesimal level, the gauge transformations (5.11) become

$$
\begin{aligned}
& \delta A=\mathrm{d} w+\mu_{2}(A, w)-\mu_{1}(v), \\
& \delta B=-\mathrm{d} v-\mu_{2}(A, v)+\mu_{2}(B, w)+\frac{1}{2} \mu_{3}(w, A, A),
\end{aligned}
$$

where $w \in H^{0}\left(U, \Omega_{X}^{0} \otimes \mathfrak{w}\right)$ and $v \in H^{0}\left(U, \Omega_{X}^{1} \otimes \mathfrak{v}\right)$. Hence, they agree with the gauge transformations in Proposition 5.3 for the 2-term $L_{\infty}$-algebra $\mathfrak{v} \stackrel{\mu_{1}}{\longrightarrow} \mathfrak{w}$ concentrated in degrees -1 and 0 .

Proof. We linearise $p=\mathrm{id}_{e}+\delta p$ and $\Lambda=\mathrm{id}_{A}+\delta \Lambda$ such that equation (5.11a) reads as

$$
\left(\mathrm{id}_{A}+\delta \Lambda\right):(A+\delta A) \otimes\left(\mathrm{id}_{e}+\delta p\right) \Rightarrow\left(\mathrm{id}_{e}+\delta p\right) \otimes A-\mathrm{d} \delta p .
$$

Identifying

$$
w=-\delta p \quad \text { and } \quad v=\delta \Lambda-\mathrm{id}_{\delta p \otimes A-\mathrm{d} \delta p}: \delta A+A \otimes \delta p-\delta p \otimes A+\mathrm{d} \delta p \Rightarrow 0,
$$

we immediately obtain the first equation in (5.12). The derivation of the second equation in (5.12) from linearising (5.11b) is somewhat more involved. We start from

$$
\begin{aligned}
(B+\delta B) & \otimes\left(\operatorname{id}_{A}+\operatorname{id}_{\delta p}\right)=\mu(A, A, \delta p)+\left[\operatorname{id}_{e} \otimes B+\operatorname{id}_{\delta p} \otimes B+\mu(\delta p, A, A)\right] \circ \\
& \circ\left[-\operatorname{did}_{A}-\mathrm{d} \delta \Lambda-\operatorname{id}_{A} \otimes \mathrm{id}_{A}-\delta \Lambda \otimes \operatorname{id}_{A}+\mu(A, w, A)\right] \circ \\
& \circ\left[-\mathrm{id}_{\mathrm{d} A}-\mathrm{id}_{\mathbf{s}(\mathrm{d} \delta \Lambda)}-\mathrm{id}_{A} \otimes \mathrm{id}_{A}-\mathrm{id}_{A} \otimes \mathrm{id}_{\mathrm{d} \delta p}-\mathrm{id}_{A} \otimes \delta \Lambda-\mathrm{id}_{\delta A} \otimes \mathrm{id}_{A}\right] .
\end{aligned}
$$

The remaining calculation is rather lengthy but straightforward, if one makes use of the (linearised) interchange law, Proposition 4.17 and the identity $\mathrm{s}(B)=-\mathrm{d} A+A \otimes A$.

\footnotetext{
${ }^{15}$ Here, $T_{p} N$ denotes the sheaf over $U \subseteq X$ with stalks $T_{p(x)} N$ over $x \in U$.
} 


\subsection{Connective structure}

Consider a semistrict principal 2-bundle $\Phi$ with a semistrict structure 2-group $\mathscr{G}=(M, N)$ over a smooth manifold $X$ with covering $\mathfrak{U}=\left\{U_{a}\right\}$. We use again the notation $\mathfrak{w}:=T_{\text {id }_{e}} M$ and $\mathfrak{v}:=\operatorname{ker}(\mathrm{t}) \subseteq T_{\mathrm{id}_{\mathrm{id}_{e}}} N$. The bundle $\Phi$ is characterised by $\mathscr{G}$-valued transition functions $\left(\left\{m_{a b}\right\},\left\{n_{a b c}\right\}\right)$. Next, we would like to equip $\Phi$ with a connective structure.

From the discussion of strict principal 2-bundles, it is clear that a connective structure will consist locally of a $\mathfrak{w}$-valued 1-form $A_{a}$, a $\mathfrak{v}$-valued 2-form $B_{a}$, and, on intersections $U_{a} \cap U_{b}$, a $T_{m_{a b}}[-1] N$-valued 1-form $\Lambda_{a b}$. On intersections of patches $U_{a} \cap U_{b},\left(A_{a}, B_{a}\right)$ and $\left(A_{b}, B_{b}\right)$ are related by a gauge transformation on $U_{a} \cap U_{b}$, which is parameterised by $\left(m_{a b}, \Lambda_{a b}\right)$. The explicit formula is then clear from Proposition 5.9 and reads as follows:

$$
\begin{aligned}
\Lambda_{a b}: \tilde{A}_{b} \otimes m_{a b} \Rightarrow & m_{a b} \otimes A-\mathrm{d} m_{a b}, \\
B_{b} \otimes \mathrm{id}_{m_{a b}}= & \mu\left(A_{b}, A_{b}, m_{a b}\right)+\left[\mathrm{id}_{m_{a b}} \otimes B_{a}+\mu\left(m_{a b}, A_{a}, A_{a}\right)\right] \circ \\
& \circ\left[-\mathrm{d} \Lambda_{a b}-\Lambda_{a b} \otimes \operatorname{id}_{A_{a}}-\mu\left(\tilde{A}_{b}, m_{a b}, A_{a}\right)\right] \circ \\
& \circ\left[-\mathrm{id}_{\mathbf{s}\left(\Lambda_{a b}\right)}-\operatorname{id}_{\tilde{A}_{b}} \otimes\left(\Lambda_{a b}+\mathrm{id}_{\mathrm{d} m_{a b}}\right)\right],
\end{aligned}
$$

provided the fake curvature $\mathcal{F}_{a}:=\mathrm{d} A_{a}+A_{a} \otimes A_{a}+\mathrm{s}\left(B_{a}\right)$ vanishes on all coordinate patches $U_{a}$.

Note that the condition that two transformations of the form (5.16) combine to a third one on non-empty triple intersection of coordinate patches yields the cocycle condition for $\left\{\Lambda_{a b}\right\}$. To derive this condition, let us consider

$$
\begin{aligned}
\Lambda_{a b}: A_{b} \otimes m_{b a} & \Rightarrow m_{b a} \otimes A_{a}-\mathrm{d} m_{b a}, \\
\Lambda_{b c}: A_{c} \otimes m_{c b} & \Rightarrow m_{c b} \otimes A_{b}-\mathrm{d} m_{c b}, \\
\Lambda_{a c}: A_{c} \otimes m_{c a} & \Rightarrow m_{c a} \otimes A_{a}-\mathrm{d} m_{c a},
\end{aligned}
$$

over a non-empty triple intersections $U_{a} \cap U_{b} \cap U_{c}$. Recall also that

$$
n_{a b c}: m_{a b} \otimes m_{b c} \Rightarrow m_{a c} .
$$

Chasing the commutative diagram relating the two possible ways of going from $\left(A_{b} \otimes m_{b a}\right) \otimes$ $m_{a c}$ to $m_{b c} \otimes A_{c}-\mathrm{d} m_{b c}$, we obtain the following proposition.

Proposition 5.11. The 1-forms $\left\{\Lambda_{a b}\right\}$ are consistent over triple overlaps $U_{a} \cap U_{b} \cap U_{c}$, if the following holds:

$$
\begin{aligned}
\Lambda_{c b} \circ & \left(\mathrm{id}_{A_{b}} \otimes n_{b a c}\right) \circ \mathrm{a}_{A_{b}, m_{b a}, m_{a c}}= \\
= & \left(n_{b a c} \otimes \mathrm{id}_{A_{c}}-\mathrm{d} n_{b a c}\right) \circ\left(\mathrm{a}_{m_{b a}, m_{a c}, A_{c}}^{-1}-\mathrm{id}_{\mathrm{d}\left(m_{b a} \otimes m_{a c}\right)}\right) \circ \\
& \circ\left(\mathrm{id}_{m_{b a}} \otimes \Lambda_{c a}-\mathrm{id} \mathrm{d}_{m_{b a} \otimes m_{a c}}\right) \circ\left(\mathrm{a}_{m_{b a}, A_{c}, m_{a c}}-\mathrm{id}_{\mathrm{d} m_{b a} \otimes m_{a c}}\right) \circ\left(\Lambda_{a b} \otimes \mathrm{id}_{m_{a c}}\right) .
\end{aligned}
$$

In the above equation, we have again used our intuitive notation: for instance, $n_{b a c} \otimes \mathrm{id}_{A_{c}}-$ $\mathrm{d} n_{b a c}$ has to be understood as

$$
n_{b a c} \otimes \operatorname{id}_{A_{c}}-\mathrm{d} n_{b a c}:\left(m_{b a} \otimes m_{a c}\right) \otimes A_{c}-\mathrm{d}\left(m_{b a} \otimes m_{a c}\right) \Rightarrow m_{b c} \otimes A_{c}-\mathrm{d} m_{b c} .
$$

We now have all the ingredients for defining the notion of a connective structure. 
Definition 5.12. A connective structure on a semistrict principal 2-bundle $\Phi$ with semistrict structure 2-group $\mathscr{G}=(M, N)$ with associated 2-term $L_{\infty}$-algebra $\mathfrak{v} \stackrel{\mu_{1}}{\longrightarrow} \mathfrak{w}$ consists of $\left(\left\{A_{a}\right\},\left\{B_{a}\right\},\left\{\Lambda_{a b}\right\}\right)$, where $A_{a} \in H^{0}\left(U_{a}, \Omega_{X}^{1} \otimes \mathfrak{w}\right), B_{a} \in H^{0}\left(U_{a}, \Omega_{X}^{2} \otimes \mathfrak{v}\right)$, and $\Lambda_{a b} \in H^{0}\left(U_{a} \cap U_{b}, \Omega_{X}^{1} \otimes T_{m_{a b}} N\right)$ such that the cocycle conditions (5.16) as well as (5.20) are satisfied, and, in addition, the 2-form fake curvature

$$
\mathcal{F}_{a}:=\mathrm{d} A_{a}+A_{a} \otimes A_{a}+\mathrm{s}\left(B_{a}\right)
$$

vanishes.

Remark 5.13. Note that by virtue of Definition 4.27 and Proposition 4.28, we can always work with a $\Lambda_{a b}^{0} \in H^{0}\left(U_{a} \cap U_{b}, \Omega_{X}^{1} \otimes \mathfrak{v}\right)$ such that

$$
\Lambda_{a b}^{0}: A_{b}-\left(m_{a b} \otimes A_{a}\right) \otimes \bar{m}_{a b}+\mathrm{d} m_{a b} \otimes \bar{m}_{a b} \Rightarrow 0
$$

instead of $\Lambda_{a b} \in H^{0}\left(U_{a} \cap U_{b}, \Omega_{X}^{1} \otimes T_{m_{a b}} N\right)$ with (5.16). Both are related by

$$
\Lambda_{a b}^{0}=\left(\Lambda_{a b} \otimes \operatorname{id}_{\bar{m}_{a b}}\right) \circ \mathrm{a}_{A_{b}, m_{a b}, \bar{m}_{a b}}^{-1}-\operatorname{id}_{\left(m_{a b} \otimes A_{a}\right) \otimes \bar{m}_{a b}-\mathrm{d} m_{a b} \otimes \bar{m}_{a b}},
$$

or, equivalently,

$$
\begin{aligned}
\Lambda_{a b}= & \mathrm{a}_{m_{a b} \otimes A_{a}+\mathrm{d} m_{a b}, \bar{m}_{a b}, m_{a b}} \circ \\
& \circ\left\{\left[\left(\Lambda_{a b}^{0}+\mathrm{id}_{\left(m_{a b} \otimes A_{a}\right) \otimes \bar{m}_{a b}-\mathrm{d} m_{a b} \otimes \bar{m}_{a b}}\right) \circ \mathrm{a}_{\left.\left.A_{b}, m_{a b}, \bar{m}_{a b}\right] \otimes \mathrm{id}_{m_{a b}}\right\} \circ}\right.\right. \\
& \circ \mathrm{a}_{A_{b} \otimes m_{a b}, \bar{m}_{a b}, m_{a b} .}^{-1}
\end{aligned}
$$

Therefore, we can say that a connective structure $\left(\left\{A_{a}\right\},\left\{B_{a}\right\},\left\{\Lambda_{a b}\right\}\right)$ is alternatively given by a tuple $\left(\left\{A_{a}\right\},\left\{B_{a}\right\},\left\{\Lambda_{a b}^{0}\right\}\right)$ in which $\Lambda_{a b}^{0}$ is as above.

Finally, we would like to describe the action of a coboundary on a connective structure $\left(\left\{A_{a}\right\},\left\{B_{a}\right\},\left\{\Lambda_{a b}\right\}\right)$. For $\left(\left\{A_{a}\right\},\left\{B_{a}\right\}\right)$ this is again clear from Proposition 5.9. For instance,

$$
\begin{aligned}
\Lambda_{a}: \tilde{A}_{a} \otimes m_{a} & \Rightarrow m_{a} \otimes A_{a}-\mathrm{d} m_{a}, \\
n_{a b}: \tilde{m}_{a b} \otimes m_{b} & \Rightarrow m_{a} \otimes m_{a b} .
\end{aligned}
$$

To derive the action on $\left\{\Lambda_{a b}\right\}$, we compare the two expressions,

$$
\begin{aligned}
& \Lambda_{a b}: A_{b} \otimes m_{b a} \Rightarrow m_{b a} \otimes A_{a}-\mathrm{d} m_{b a}, \\
& \tilde{\Lambda}_{a b}: \tilde{A}_{b} \otimes \tilde{m}_{b a} \Rightarrow \tilde{m}_{b a} \otimes \tilde{A}_{a}-\mathrm{d} \tilde{m}_{b a} .
\end{aligned}
$$

Again, chasing the corresponding commutative diagram relating the two possible ways of going from $\left(\tilde{A}_{a} \otimes m_{a}\right) \otimes m_{a b}$ to $\left(\tilde{m}_{a b} \otimes \tilde{A}_{b}\right) \otimes m_{b}-\mathrm{d} \tilde{m}_{a b} \otimes m_{b}$ yields the following proposition.

Proposition 5.14. The 1-forms $\left\{\Lambda_{a b}\right\}$ and $\left\{\tilde{\Lambda}_{a b}\right\}$ of two equivalent connective structures $\left(\left\{A_{a}\right\},\left\{B_{a}\right\},\left\{\Lambda_{a b}\right\}\right)$ and $\left(\left\{\tilde{A}_{a}\right\},\left\{\tilde{B}_{a}\right\},\left\{\tilde{\Lambda}_{a b}\right\}\right)$ on a semistrict principal 2-bundle $\Phi$ with semistrict structure 2-group are related by

$$
\begin{aligned}
& \left(\tilde{\Lambda}_{b a} \otimes \mathrm{id}_{m_{b}}\right) \circ \mathrm{a}_{\tilde{A}_{a}, \tilde{m}_{a b}, m_{b}}^{-1} \circ\left(\mathrm{id}_{\tilde{A}_{a}} \otimes n_{a b}\right) \circ \mathrm{a}_{\tilde{A}_{a}, m_{a}, m_{a b}}= \\
& =\left(\mathrm{a}_{\tilde{m}_{a b}, \tilde{A}_{b}, m_{b}}^{-1}-\mathrm{id}_{\mathrm{d} \tilde{m}_{a b} \otimes m_{b}}\right) \circ\left(\mathrm{id}_{\tilde{m}_{a b}} \otimes \Lambda_{b}^{-1}-\mathrm{id}_{\mathrm{d} \tilde{m}_{a b} \otimes m_{b}}\right) \circ\left(\mathrm{a}_{m_{a b}, m_{b}, A_{b}}-\mathrm{id}_{\mathrm{d}\left(\tilde{m}_{a b} \otimes m_{b}\right)}\right) \circ \\
& \quad \circ\left(n_{a b}^{-1} \otimes \mathrm{id}_{A_{b}}-\mathrm{d} n_{a b}^{-1}\right) \circ\left(\mathrm{a}_{m_{a}, m_{a b}, A_{b}}^{-1}-\mathrm{id}_{\mathrm{d}\left(m_{a} \otimes m_{a b}\right)}\right) \circ\left(\mathrm{id}_{m_{a}} \otimes \Lambda_{b a}-\mathrm{id}_{\mathrm{d} m_{a} \otimes m_{a b}}\right) \circ \\
& \quad \circ\left(\mathrm{a}_{m_{a}, A_{a}, m_{a b}}-\mathrm{id}_{\mathrm{d} m_{a} \otimes m_{a b}}\right) \circ\left(\Lambda_{a} \otimes \mathrm{id}_{m_{a b}}\right) .
\end{aligned}
$$

As before, we have used our intuitive notation here. 


\subsection{Semistrict non-Abelian Deligne cohomology}

Deligne cohomology describes gauge configurations on a principal bundle with connection modulo gauge transformations, which act simultaneously on the connection and the transition functions of the bundle. Deligne cohomology for categorified bundles was described previously in some special cases. In particular, the case of Abelian gerbes was discussed in [43], the case of principal 2-bundles with strict structure 2-group was given in [44], and the case of principal 3-bundles was presented in [10] (see also [45]). Here, we wish to describe the low-lying sets of the Deligne cohomology with values in a semistrict Lie 2-group. In the special case of the 2-group BU(1), this reduces to ordinary, Abelian Deligne cohomology.

As before, we consider a smooth manifold $X$ with covering $\mathfrak{U}=\left\{U_{a}\right\}$. We shall write $C^{p, q}(\mathfrak{U}, \mathcal{S})$ for the $\Omega_{X}^{q} \otimes \mathcal{S}$-valued Čech $p$-cochains relative to the covering $\mathfrak{U}$, where $\mathcal{S}$ is a some sheaf on $X$. Now, let $\mathscr{G}=(M, N)$ be a semistrict Lie 2-group. We again make use of the notation $\mathfrak{w}:=T_{\mathrm{id}_{e}} M$ and $\mathfrak{v}:=\operatorname{ker}(\mathrm{t}) \subseteq T_{\mathrm{id}_{\mathrm{id}_{e}}} N$ and denote the corresponding 2-term $L_{\infty}$-algebra by $\mathfrak{v} \stackrel{\mu_{1}}{\longrightarrow} \mathfrak{w}$.

Definition 5.15. Let $\mathscr{G}=(M, N)$ be a semistrict Lie 2-group with underlying 2-term $L_{\infty}$-algebra $\mathfrak{v} \stackrel{\mu_{1}}{\longrightarrow} \mathfrak{w}$. A $\mathscr{G}$-valued degree- $p$ Deligne cochain consists of elements

$$
\begin{aligned}
\left(\left\{n_{a_{0} \cdots a_{p}}\right\}, \ldots,\left\{n_{a_{0}}\right\}\right) & \in C^{p, 0}(\mathfrak{U}, N) \times C^{p-1,1}(\mathfrak{U}, \mathfrak{v}) \times \cdots \times C^{0, p}(\mathfrak{U}, \mathfrak{v}), \\
\left(\left\{m_{a_{0} \cdots a_{p-1}}\right\}, \ldots,\left\{m_{a_{0}}\right\}\right) & \in C^{p-1,0}(\mathfrak{U}, M) \times C^{p-2,1}(\mathfrak{U}, \mathfrak{w}) \times \cdots \times C^{0, p-1}(\mathfrak{U}, \mathfrak{w}) .
\end{aligned}
$$

The sum of the Čech and de Rham degrees of $\left(\left\{n_{a_{0} \cdots a_{p}}\right\}, \ldots,\left\{n_{a_{0}}\right\}\right)$ is $p$ while for $\left(\left\{m_{a_{0} \cdots a_{p-1}}\right\}, \ldots,\left\{m_{a_{0}}\right\}\right)$ it is $p-1$. Compared to the analogous discussions of Deligne cochains for strict 2-groups in Schreiber \& Waldorf [44], we have dropped Cech cochains that are always cohomologous to trivial ones, cf. [10] and Proposition 3.15.

Using our results from the previous sections as well as appendix B, we can describe Deligne cohomology with semistrict 2-groups up to degree 2. In particular, we have provided ample motivation for giving the following definition.

Definition 5.16. A degree- $p$ Deligne cocycle is a degree-p Deligne cochain satisfying a cocycle relation. Here, we restrict ourselves to the case $p \leq 2$, and define the following:

(i) A degree-0 Deligne cocycle is an element $\left\{n_{a}\right\} \in C^{0,0}(\mathfrak{U}, N)$ such that on non-empty intersections $U_{a} \cap U_{b}$

$$
n_{a}=n_{b} .
$$

(ii) A degree-1 Deligne cocycle consists of elements $\left\{n_{a b}\right\} \in C^{1,0}(\mathfrak{U}, N),\left\{B_{a}\right\} \in$ $C^{0,1}(\mathfrak{U}, \mathfrak{v})$, and $\left\{m_{a}\right\} \in C^{0,0}(\mathfrak{U}, M)$ such that on relevant non-empty intersections of coordinate patches

$$
n_{a b}: m_{b} \Rightarrow m_{a}, \quad n_{a b} \circ n_{b c}=n_{a c}
$$

and $d^{16}$

$$
B_{b}=\left(n_{a b}^{-1} \circ B_{a} \circ n_{a b}\right) \circ\left(n_{a b}^{-1} \circ\left(-\mathrm{d} n_{a b}\right)\right) .
$$

\footnotetext{
${ }^{16}$ Here, the operations $\circ$ are defined in a detailed discussion of these relations in appendix B.
} 
(iii) A degree-2 Deligne cocycle consists of elements $\left\{n_{a b c}\right\} \in C^{2,0}(\mathfrak{U}, N)$ and $\left\{m_{a b}\right\} \in$ $C^{1,0}(\mathfrak{U}, M)$ such that on the relevant non-empty intersections of coordinate patches

$$
\begin{gathered}
n_{a b c}: m_{a b} \otimes m_{b c} \Rightarrow m_{a c}, \\
n_{a c d} \circ\left(n_{a b c} \otimes \operatorname{id}_{m_{c d}}\right) \circ \mathrm{a}_{m_{a b}, m_{b c}, m_{c d}}=n_{a b d} \circ\left(\operatorname{id}_{m_{a b}} \otimes n_{b c d}\right),
\end{gathered}
$$

elements $\left\{A_{a}\right\} \in C^{0,1}(\mathfrak{U}, \mathfrak{w})$ and $\left\{B_{a}\right\} \in C^{0,2}(\mathfrak{U}, \mathfrak{v})$ such that

$$
\mathrm{d} A_{a}+A_{a} \otimes A_{a}+\mathrm{s}\left(B_{a}\right)=0,
$$

and elements $\left\{\Lambda_{a b}^{0}\right\} \in C^{1,1}(\mathfrak{U}, \mathfrak{v})$ such that

$$
\Lambda_{a b}^{0}: A_{b}-\left(m_{a b} \otimes A_{a}\right) \otimes \bar{m}_{a b}+\mathrm{d} m_{a b} \otimes \bar{m}_{a b} \Rightarrow 0,
$$

or, equivalently,

$$
\Lambda_{a b}: A_{b} \otimes m_{a b} \Rightarrow m_{a b} \otimes A_{a}-\mathrm{d} m_{a b}
$$

with

$$
\begin{aligned}
\Lambda_{a b}:= & \mathrm{a}_{m_{a b} \otimes A_{a}+\mathrm{d} m_{a b}, \bar{m}_{a b}, m_{a b}} \circ \\
& \circ\left\{\left[\left(\Lambda_{a b}^{0}+\mathrm{id}_{\left.\left(m_{a b} \otimes A_{a}\right) \otimes \bar{m}_{a b}-\mathrm{d} m_{a b} \otimes \bar{m}_{a b}\right)}\right) \circ \mathrm{a}_{A_{b}, m_{a b}, \bar{m}_{a b}}\right] \otimes \mathrm{id}_{m_{a b}}\right\} \circ \\
& \circ \mathrm{a}_{A_{b} \otimes m_{a b}, \bar{m}_{a b}, m_{a b}}^{-1}
\end{aligned}
$$

such that

$$
\begin{aligned}
\Lambda_{c b} & \circ\left(\mathrm{id}_{A_{b}} \otimes n_{b a c}\right) \circ \mathrm{a}_{A_{b}, m_{b a}, m_{a c}}= \\
= & \left(n_{b a c} \otimes \mathrm{id}_{A_{c}}-\mathrm{d} n_{b a c}\right) \circ\left[\mathrm{a}_{m_{b a}, m_{a c}, A_{c}}^{-1}-\mathrm{id}_{\mathrm{d}\left(m_{b a} \otimes m_{a c}\right)}\right] \circ \\
\quad & \quad\left(\mathrm{id}_{m_{b a}} \otimes \Lambda_{c a}-\mathrm{id}_{\mathrm{d} m_{b a} \otimes m_{a c}}\right) \circ\left(\mathrm{a}_{m_{b a}, A_{c}, m_{a c}}-\mathrm{id}_{\mathrm{d} m_{b a} \otimes m_{a c}}\right) \circ\left(\Lambda_{a b} \otimes \mathrm{id}_{m_{a c}}\right),
\end{aligned}
$$

and

$$
\begin{aligned}
B_{b} \otimes \mathrm{id}_{m_{a b}}= & \mu\left(A_{b}, A_{b}, m_{a b}\right)+\left[\operatorname{id}_{m_{a b}} \otimes B_{a}+\mu\left(m_{a b}, A_{a}, A_{a}\right)\right] \circ \\
& \circ\left[-\mathrm{d} \Lambda_{a b}-\Lambda_{a b} \otimes \operatorname{id}_{A_{a}}-\mu\left(A_{b}, m_{a b}, A_{a}\right)\right] \circ \\
& \circ\left[-\mathrm{id}_{\mathbf{s}\left(\Lambda_{a b}\right)}-\operatorname{id}_{A_{b}} \otimes\left(\Lambda_{a b}+\mathrm{id}_{\mathrm{d} m_{a b}}\right)\right] .
\end{aligned}
$$

Furthermore, we need to state what we would like to understand by Deligne coboundary transformations.

Definition 5.17. Two degree-p Deligne cocycles are called cohomologous or equivalent if and only if there is a degree- $(p-1)$ Deligne cochain relating both. In more detail, we define the following:

(i) Two degree-1 Deligne cocycles $\left(\left\{n_{a b}\right\},\left\{B_{a}\right\},\left\{m_{a}\right\}\right)$ and $\left(\left\{\tilde{n}_{a b}\right\},\left\{\tilde{B}_{a}\right\},\left\{\tilde{m}_{a}\right\}\right)$ are called cohomologous if and only if there is a degree-0 Deligne cochain $\left\{n_{a}\right\} \in C^{0,0}(\mathfrak{U}, N)$ such that on the relevant non-empty intersections of coordinate patches

$$
n_{a}: \tilde{m}_{a} \Rightarrow m_{a} \quad \text { and } \quad n_{a b}=n_{a} \circ \tilde{n}_{a b} \circ n_{b}^{-1}
$$

and

$$
\tilde{B}_{a}=\left(n_{a}^{-1} \circ B_{a} \circ n_{a}\right) \circ\left(n_{a}^{-1} \circ\left(-\mathrm{d} n_{a}\right)\right) .
$$


(ii) Two degree-2 Deligne cocycles $\left(\left\{m_{a b}\right\},\left\{n_{a b c}\right\},\left\{A_{a}\right\},\left\{B_{a}\right\},\left\{\Lambda_{a b}^{0}\right\}\right)$ and $\left(\left\{\tilde{m}_{a b}\right\},\left\{\tilde{n}_{a b c}\right\}\right.$, $\left.\left\{\tilde{A}_{a}\right\},\left\{\tilde{B}_{a}\right\},\left\{\tilde{\Lambda}_{a b}^{0}\right\}\right)$ are called cohomologous if and only if there is a degree-1 Deligne cochain $\left(\left\{n_{a b}\right\},\left\{\Lambda_{a}\right\},\left\{m_{a}\right\}\right)$ such that on the relevant non-empty intersections of coordinate patches

$$
\begin{aligned}
n_{a b}: \tilde{m}_{a b} \otimes m_{b} \Rightarrow & m_{a} \otimes m_{a b}, \\
n_{a c} \circ\left(\tilde{n}_{a b c} \otimes \operatorname{id}_{m_{c}}\right)= & \left(\operatorname{id}_{m_{a}} \otimes n_{a b c}\right) \circ \mathrm{a}_{m_{a}, m_{a b}, m_{b c}} \circ\left(n_{a b} \otimes \operatorname{id}_{m_{b c}}\right) \circ \\
& \circ \mathrm{a}_{\tilde{m}_{a b}, m_{b}, \tilde{m}_{b c}}^{-1} \circ\left(\operatorname{id}_{\tilde{m}_{a b}} \otimes n_{b c}\right) \circ \mathrm{a}_{\tilde{m}_{a b}, \tilde{m}_{b c}, m_{c}},
\end{aligned}
$$

and

$$
\Lambda_{a}^{0}: \tilde{A}_{a}-\left(m_{a} \otimes A_{a}\right) \otimes \bar{m}_{a}+\mathrm{d} m_{a} \otimes \bar{m}_{a} \Rightarrow 0,
$$

or, equivalently,

$$
\Lambda_{a}: \tilde{A}_{a} \otimes m_{a} \Rightarrow m_{a} \otimes A_{a}-\mathrm{d} m_{a}
$$

with

$$
\begin{aligned}
\Lambda_{a}:= & \mathrm{a}_{m_{a} \otimes A_{a}+\mathrm{d} m_{a}, \bar{m}_{a}, m_{a}} \circ \\
& \circ\left\{\left[\left(\Lambda_{a}^{0}+\mathrm{id}_{\left(m_{a} \otimes A_{a}\right) \otimes \bar{m}_{a}-\mathrm{d} m_{a} \otimes \bar{m}_{a}}\right) \circ \mathrm{a}_{\tilde{A}_{a}, m_{a}, \bar{m}_{a}}\right] \otimes \mathrm{id}_{m_{a}}\right\} \circ \\
& \circ \mathrm{a}_{\tilde{A}_{a} \otimes m_{a}, \bar{m}_{a}, m_{a}}^{-1}
\end{aligned}
$$

such that

$$
\begin{aligned}
\tilde{B}_{a} \otimes \mathrm{id}_{m_{a}}= & \mu\left(\tilde{A}_{a}, \tilde{A}_{a}, m_{a}\right)+\left[\mathrm{id}_{m_{a}} \otimes B_{a}+\mu\left(m_{a}, A_{a}, A_{a}\right)\right] \circ \\
& \circ\left[-\mathrm{d} \Lambda_{a}-\Lambda_{a} \otimes \mathrm{id}_{A}-\mu\left(\tilde{A}_{a}, m_{a}, A_{a}\right)\right] \circ \\
& \circ\left[-\mathrm{id}_{\mathbf{s}\left(\mathrm{d}_{a}\right)}-\mathrm{id}_{\tilde{A}_{a}} \otimes\left(\Lambda_{a}+\mathrm{id}_{\mathrm{d} m_{a}}\right)\right],
\end{aligned}
$$

$$
\begin{aligned}
& \left(\tilde{\Lambda}_{b a} \otimes \mathrm{id}_{m_{b}}\right) \circ \mathrm{a}_{\tilde{A}_{a}, \tilde{m}_{a b}, m_{b}}^{-1} \circ\left(\mathrm{id}_{\tilde{A}_{a}} \otimes n_{a b}\right) \circ \mathrm{a}_{\tilde{A}_{a}, m_{a}, m_{a b}}= \\
& =\left(\mathrm{a}_{\tilde{m}_{a b}, \tilde{A}_{b}, m_{b}}^{-1}-\mathrm{id}_{\mathrm{d} \tilde{m}_{a b} \otimes m_{b}}\right) \circ\left(\mathrm{id}_{\tilde{m}_{a b}} \otimes \Lambda_{b}^{-1}-\mathrm{id}_{\mathrm{d} \tilde{m}_{a b} \otimes m_{b}}\right) \circ\left(\mathrm{a}_{m_{a b}, m_{b}, A_{b}}-\mathrm{id}_{\mathrm{d}\left(\tilde{m}_{a b} \otimes m_{b}\right.}\right) \circ \\
& \quad \circ\left(n_{a b}^{-1} \otimes \mathrm{id}_{A_{b}}-\mathrm{d} n_{a b}^{-1}\right) \circ\left(\mathrm{a}_{m_{a}, m_{a b}, A_{b}}^{-1}-\mathrm{id}_{\mathrm{d}\left(m_{a} \otimes m_{a b}\right)}\right) \circ\left(\mathrm{id}_{m_{a}} \otimes \Lambda_{b a}-\mathrm{id}_{\mathrm{d} m_{a} \otimes m_{a b}}\right) \circ \\
& \quad \circ\left(\mathrm{a}_{m_{a}, A_{a}, m_{a b}}-\mathrm{id}_{\mathrm{d} m_{a} \otimes m_{a b}}\right) \circ\left(\Lambda_{a} \otimes \mathrm{id}_{m_{a b}}\right) .
\end{aligned}
$$

Note that there are further equivalences between Deligne coboundaries arising from modification transformations. These are not relevant for our discussion of Deligne cohomology and we therefore do not wish to go into any further detail.

Let us end this section by briefly commenting on the interpretation of elements of Deligne cohomology sets. The first case of degree- 0 Deligne cocycles is readily understood. A degree-0 Deligne cocycles describes an $N$-valued function on $X$, which could be regarded as a principal 0-bundle.

The case of Deligne 1-cocycles is slightly more involved. If $N$ is a group, then a degree-1 Deligne cocycle defines a principal (1-)bundle with connection one-form $B$ and a preferred section $m$. This data was called a crossed module bundle, from which crossed module bundle gerbes were constructed in [2], see also [46, 47]. Recall that an Abelian bundle $(p+1)$-gerbe over a manifold $X$ can be constructed from the notion of an Abelian bundle 
$p$-gerbe, by considering a surjective submersion $Y \rightarrow X$ together with Abelian bundle $p$ gerbes over $Y \times_{X} Y$. The analogous construction for crossed module bundle gerbes starts from a crossed module bundle. If $N$ is not a group, then a Deligne 1-cocycle describes a 2group principal bundle, which is a special form of a groupoid principal bundle. Considering 2-group principal bundles over $Y \times_{X} Y$ yields then to 2-group bundle gerbes or the principal 2-bundles described by Deligne 2-cocycles.

A degree-2 Deligne cocycle describes a semistrict principal 2-bundle with connective structure. Again, gauge equivalence is captured by the cohomology. To study such Deligne 2 -cocycles further, it is useful to introduce the curvature 3 -form, apart from the 2 -form fake curvature (5.22) that vanishes; see also Proposition 5.3.

Definition 5.18. Let $\left(\left\{A_{a}\right\},\left\{B_{a}\right\},\left\{\Lambda_{a b}\right\}\right)$ be a connective structure on a semistrict principal 2-bundle $\Phi$. The associated 3-form curvature is defined as follows:

$$
H_{a}:=\mathrm{d} B_{a}+\mathrm{id}_{A_{a}} \otimes B_{a}-B_{a} \otimes \mathrm{id}_{A_{a}}+\mu\left(A_{a}, A_{a}, A_{a}\right) .
$$

\section{Application: Penrose-Ward transform}

As an application of the theory of principal 2-bundles which we have developed in the previous sections, we now show how to generalise the results of [9]. Specifically, [9] established a Penrose-Ward transform that yields a bijection between holomorphic principal 2-bundles with strict structure 2-group over a twistor space and non-Abelian self-dual tensor fields on six-dimensional flat space-time. We can now replace the strict principal 2-bundles by semistrict ones in this construction.

In the following, we denote by $\mathcal{O}_{X}$ the sheaf of holomorphic functions and by $\Omega_{X}^{p}$ the sheaf of holomorphic differential $p$-forms on a complex (super)manifold $X$.

\subsection{Supertwistor space}

The twistor space $P^{6}$ underlying chiral field theories on flat complexified six-dimensional space-time $\mathbb{C}^{6}$ is the moduli space of $\alpha$-planes or self-dual 3-planes in $\mathbb{C}^{6}$. This twistor space has been described in great detail before [11, 12, 48-53], and its supersymmetric extension $P^{6 \mid 2 n}$ was discussed in $[9,10,54]$. We therefore keep our following exposition brief.

The starting point is the chiral superspace $M^{6 \mid 8 n}:=\mathbb{C}^{6 \mid 8 n}$ with $n=0,1,2$. This space can be equipped with the coordinates $\left(x^{A B}, \eta_{I}^{A}\right)$, where $x^{A B}=-x^{B A}$ with $A, B, \ldots=$ $1, \ldots, 4$ are the usual Graßmann-even coordinates in spinor notation, $\eta_{I}^{A}$ are the Graßmannodd coordinates and $I, J, \ldots=1, \ldots, 2 n$ are the R-symmetry indices. We may raise and lower the spinor indices using the Levi-Civita symbol, that is, $x_{A B}=\frac{1}{2} \varepsilon_{A B C D} x^{C D} \Leftrightarrow x^{A B}=$ $\frac{1}{2} \varepsilon^{A B C D} x_{C D}$. Note that in the real setting, the R-symmetry group of the superconformal group $\operatorname{OSp}(2,6 \mid 2 n)$ is

$$
\operatorname{Sp}(n)=\left\{\begin{array}{ll}
\operatorname{Sp}(1) \cong \mathrm{SU}(2) & \text { for } n=1 \\
\mathrm{Sp}(2) \cong \mathrm{USp}(4) \subset \mathrm{Sp}(4, \mathbb{C}) & \text { for } n=2
\end{array} .\right.
$$


The group $\operatorname{Sp}(n)$ is defined as the elements of $\mathrm{SU}(2 n)$ leaving an antisymmetric $2 n \times 2 n$ matrix $\Omega$ invariant, which we can fix according to

$$
\Omega=\operatorname{diag}(\underbrace{\varepsilon, \ldots, \varepsilon}_{n-\text { times }}) \text { with } \quad \varepsilon:=\left(\begin{array}{cc}
0 & 1 \\
-1 & 0
\end{array}\right) .
$$

However, working in the complex setting, we shall employ appropriate complexifications of the above groups.

We further introduce the superspace derivatives

$$
P_{A B}:=\frac{\partial}{\partial x^{A B}} \quad \text { and } \quad D_{A}^{I}:=\frac{\partial}{\partial \eta_{I}^{A}}-2 \Omega^{I J} \eta_{J}^{B} \frac{\partial}{\partial x^{A B}},
$$

which obey

$$
\left\{D_{A}^{I}, D_{B}^{J}\right\}=-4 \Omega^{I J} P_{A B} .
$$

Next, we let $\mathbb{P}^{3}$ be the complex projective 3 -space and define the correspondence space $F^{9 \mid 8 n}:=\mathbb{C}^{6 \mid 8 n} \times \mathbb{P}^{3}$. It can be equipped with coordinates $\left(x^{A B}, \eta_{I}^{A}, \lambda_{A}\right)$ where $\lambda_{A}$ are homogeneous coordinates on $\mathbb{P}^{3}$. On the correspondence space, we introduce the twistor distribution, denoted by $D$, which is an integrable distribution of rank $3 \mid 6 n$ generated by the vector fields

$$
D:=\operatorname{span}\left\{V^{A}, V^{I A B}\right\} \quad \text { with } \quad V^{A}:=\lambda_{B} \partial^{A B} \quad \text { and } \quad V^{I A B}:=\frac{1}{2} \varepsilon^{A B C D} \lambda_{C} D_{D}^{I} .
$$

The supertwistor space $P^{6 \mid 2 n}$ is then defined to be the associated leaf space $P^{6 \mid 2 n}:=$ $F^{9 \mid 8 n} / D$. We can now establish a twistor correspondence which is captured by the double fibration

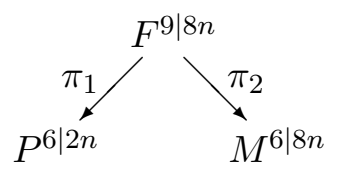

where $\pi_{2}$ is the trivial projection, while

$$
\pi_{1}:\left(x^{A B}, \eta_{I}^{A}, \lambda_{A}\right) \mapsto\left(z^{A}, \eta_{I}, \lambda_{A}\right)=\left(\left(x^{A B}+\Omega^{I J} \eta_{I}^{A} \eta_{J}^{B}\right) \lambda_{B}, \eta_{I}^{A} \lambda_{A}, \lambda_{A}\right)
$$

contains the so-called incidence relation

$$
z^{A}=\left(x^{A B}+\Omega^{I J} \eta_{I}^{A} \eta_{J}^{B}\right) \lambda_{B} \quad \text { and } \quad \eta_{I}=\eta_{I}^{A} \lambda_{A} .
$$

This incidence relation yields a geometric correspondence between points $x \in M^{6 \mid 8 n}$ and complex projective 3 -spaces $\hat{x}=\pi_{1}\left(\pi_{2}^{-1}(x)\right) \hookrightarrow P^{6 \mid 2 n}$ as well as points $p \in P^{6 \mid 2 n}$ in twistor space and $3 \mid 6 n$-superplanes $\pi_{2}\left(\pi_{1}^{-1}(p)\right) \hookrightarrow M^{6 \mid 8 n}$ which is a supersymmetric extension of a totally null 3-plane in $\mathbb{C}^{6}$. It also follows that $P^{6 \mid 2 n}$ the quadric hypersurface given by the zero locus

$$
z^{A} \lambda_{A}-\Omega^{I J} \eta_{I} \eta_{J}=0
$$

inside the total space of the holomorphic fibration $\mathbb{C}^{4 \mid 2 n} \otimes \mathcal{O}_{\mathbb{P}^{3}}(1) \rightarrow \mathbb{P}^{3}$ with fibre coordinates $z^{A}$ and $\eta_{I}$ as well as base coordinates $\lambda_{A}$. 
Remark 6.1. In our subsequent discussion, we shall always choose the standard Stein cover $\hat{\mathfrak{U}}=\left\{\hat{U}_{a}\right\}$ on the twistor space $P^{6 \mid 2 n} \rightarrow \mathbb{P}^{3}$ (generated by the standard Stein cover on $\left.\mathbb{P}^{3}\right)$ and the induced cover $\mathfrak{U}^{\prime}:=\left\{U_{a}^{\prime}:=\pi_{1}^{-1}\left(U_{a}\right)\right\}$ on the correspondence space $F^{9 \mid 8 n}$, respectively.

\subsection{Penrose-Ward transform}

To formulate the Penrose-Ward transform, we first need to introduce a few basic notions. In particular, we will need the sheaf of holomorphic relative differential $p$-forms, denoted by $\Omega_{\pi_{1}}^{p}$, on $F^{9 \mid 8 n}$ along the fibration $\pi_{1}: F^{9 \mid 8 n} \rightarrow P^{6 \mid 2 n}$. It is defined by the short exact sequence

$$
0 \longrightarrow \pi_{1}^{*} \Omega_{P^{6 \mid 2 n}}^{1} \wedge \Omega_{F^{9 \mid 8 n}}^{p-1} \longrightarrow \Omega_{F^{9 \mid 8 n}}^{p} \longrightarrow \Omega_{\pi_{1}}^{p} \longrightarrow 0 .
$$

In addition, if $\operatorname{pr}_{\pi_{1}}: \Omega_{F 9 \mid 8 n}^{p} \rightarrow \Omega_{\pi_{1}}^{p}$ denotes the quotient mapping, we can define the relative exterior derivative $\mathrm{d}_{\pi_{1}}$ by setting

$$
\mathrm{d}_{\pi_{1}}:=\operatorname{pr}_{\pi_{1}} \circ \mathrm{d}: \Omega_{\pi_{1}}^{p} \rightarrow \Omega_{\pi_{1}}^{p+1},
$$

where $\mathrm{d}$ denotes the usual holomorphic exterior derivative on the correspondence space. In the local coordinates $\left(x^{A B}, \eta_{I}^{A} \lambda_{A}\right)$ on $F^{9 \mid 8 n}, \mathrm{~d}_{\pi_{1}}$ is presented in terms of the vector fields of the twistor distribution (6.5); see also (6.21) below. The relative exterior derivative characterises the so-called relative holomorphic de Rham complex, which is the complex that is given in terms of an injective resolution of the topological inverse $\pi_{1}^{-1} \mathcal{O}_{P^{6 \mid 2 n}}$ of the sheaf $\mathcal{O}_{P^{6 \mid 2 n}}$ on the correspondence space $F^{9 \mid 8 n}$ :

$$
0 \longrightarrow \pi_{1}^{-1} \mathcal{O}_{P^{6 \mid 2 n}} \longrightarrow \mathcal{O}_{F^{9 \mid 8 n}} \stackrel{\mathrm{d}_{\pi_{1}}}{\longrightarrow} \Omega_{\pi_{1}}^{1} \stackrel{\mathrm{d}_{\pi_{1}}}{\longrightarrow} \Omega_{\pi_{1}}^{2} \stackrel{\mathrm{d}_{\pi_{1}}}{\longrightarrow} \cdots
$$

Note that $\pi_{1}^{-1} \mathcal{O}_{P^{6 \mid 2 n}}$ consists of those holomorphic functions that are locally constant along the fibres of $\pi_{1}: F^{9 \mid 8 n} \rightarrow P^{6 \mid 2 n}$.

Next, let $\Phi^{\prime}$ be a holomorphic semistrict principal 2-bundles on the correspondence space $F^{9 \mid 8 n}$, with $\mathscr{G}=(M, N)$ as its semistrict structure 2-group. As before, we denote the 2-term $L_{\infty}$-algebra associated with $\mathscr{G}$ by $\mathfrak{v} \stackrel{\mu_{1}}{\longrightarrow} \mathfrak{w}$, where $\mathfrak{w}:=T_{\mathrm{id}_{e}} M$ and $\mathfrak{v}:=$ $\operatorname{ker}(\mathrm{t}) \subseteq T_{\mathrm{id}_{\mathrm{id}_{e}}} N$. The bundle $\Phi^{\prime}$ is described by holomorphic $\mathscr{G}$-valued transition functions $\left(\left\{m_{a b}^{\prime}\right\},\left\{n_{a b c}^{\prime}\right\}\right)$ relative to the cover $\mathfrak{U}^{\prime}$.

As we shall see momentarily, the Penrose-Ward transform will be based on so-called relative degree-2 Deligne cohomology. For this reason, we wish to equip $\Phi^{\prime}$ with a relative connective structure and study its behaviour under equivalence transformations. Concretely, $\Phi^{\prime}$ is then described by a degree-2 Deligne cocycle ${ }^{17}$

$$
\left(\left\{m_{a b}^{\prime}\right\},\left\{n_{a b c}^{\prime}\right\},\left\{A_{a}^{\prime}\right\},\left\{B_{a}^{\prime}\right\},\left\{\Lambda_{a b}^{\prime}\right\}\right)
$$

with $\left\{m_{a b}^{\prime}\right\} \in C_{\pi_{1}}^{1,0}\left(\mathfrak{U}^{\prime}, M\right),\left\{n_{a b c}^{\prime}\right\} \in C_{\pi_{1}}^{2,0}\left(\mathfrak{U}^{\prime}, N\right),\left\{\Lambda_{a b}^{\prime}\right\} \in C_{\pi_{1}}^{1,1}\left(\mathfrak{U}^{\prime}, \mathfrak{v}\right),\left\{A_{a}^{\prime}\right\} \in C_{\pi_{1}}^{0,1}\left(\mathfrak{U}^{\prime}, \mathfrak{w}\right)$, and $\left\{B_{a}^{\prime}\right\} \in C_{\pi_{1}}^{0,2}\left(\mathfrak{U}^{\prime}, \mathfrak{v}\right)$. Here, the subscript ' $\pi_{1}$ ' indicates that these are relative differential forms. For instance, the $\Lambda_{a b}^{\prime}$ and $A_{a}^{\prime}$ take values in $\Omega_{\pi_{1}}^{1} \otimes \mathfrak{v}$ and $\Omega_{\pi_{1}}^{1} \otimes \mathfrak{w}$, respectively, while

\footnotetext{
${ }^{17}$ To simplify notation, we shall suppress the superscript 0 in the $\Lambda$-part of the cocycle here and in the following.
} 
the $B_{a}^{\prime}$ take values in $\Omega_{\pi_{1}}^{2} \otimes \mathfrak{v}$. In addition, we call the relative connective structure flat whenever, apart from the vanishing of 2 -form fake curvature,

$$
\mathcal{F}_{a}^{\prime}=\mathrm{d}_{\pi_{1}} A_{a}^{\prime}+\frac{1}{2} \mu_{2}\left(A_{a}^{\prime}, A_{a}^{\prime}\right)-\mu_{1}\left(B_{a}^{\prime}\right)=0
$$

inherent to 2-degree Deligne cocycles, also the 3-form curvature vanishes

$$
H_{a}^{\prime}=\mathrm{d}_{\pi_{1}} B_{a}^{\prime}+\mu_{2}\left(A_{a}^{\prime}, B_{a}^{\prime}\right)-\frac{1}{3 !} \mu_{3}\left(A_{a}^{\prime}, A_{a}^{\prime}, A_{a}^{\prime}\right)=0 .
$$

The final ingredient we shall need is a holomorphic semistrict principal 2-bundle $\hat{\Phi}$ on $P^{6 \mid 2 n}$ with $\mathscr{G}=(M, N)$ as its semistrict structure 2-group. The bundle $\hat{\Phi}$ is described by holomorphic $\mathscr{G}$-valued transition functions $\left(\left\{\hat{m}_{a b}\right\},\left\{\hat{n}_{a b c}\right\}\right)$ relative to the cover $\hat{\mathfrak{U}}$. Following Manin [55], $\hat{\Phi}$ will be called $M^{6 \mid 8 n}$-trivial whenever it is holomorphically trivial on $\hat{x}=\pi_{1}\left(\pi_{2}^{-1}(x)\right) \hookrightarrow P^{6 \mid 2 n}$ for all $x \in M^{6 \mid 8 n}$; see also Definition 3.19.

Then we have the following result.

Proposition 6.2. Consider $\pi_{1}: F^{9 \mid 8 n} \rightarrow P^{6 \mid 2 n}$ of the double fibration (6.6). There is a bijection between

(i) equivalence classes of topologically trivial $M^{6 \mid 8 n}$-trivial holomorphic semistrict principal 2-bundles on $P^{6 \mid 2 n}$ and

(ii) equivalence classes of holomorphically trivial semistrict principal 2-bundles on $F^{9 \mid 8 n}$ equipped with a relative connective structure which is globally flat.

Proof. (i) $\rightarrow$ (ii) Let $\hat{\Phi}$ be an $M^{6 \mid 8 n}$-trivial holomorphic semistrict principal 2-bundle on the twistor space $P^{6 \mid 2 n}$ described by holomorphic transition functions $\left(\left\{\hat{m}_{a b}\right\},\left\{\hat{n}_{a b c}\right\}\right)$. Furthermore, let $\Phi^{\prime}=\pi_{1}^{*} \hat{\Phi}$ be its pullback to the correspondence space $F^{9 \mid 8 n}$; see also Definition 3.18. It is described by holomorphic transition functions $\left(\left\{m_{a b}^{\prime}\right\},\left\{n_{a b c}^{\prime}\right\}\right)$ which are annihilated by the relative exterior derivative $d_{\pi_{1}}$. More precisely, it is described by the relative degree-2 Deligne cocycle

$$
\left(\left\{m_{a b}^{\prime}=\pi_{1}^{*} \hat{m}_{a b}\right\},\left\{n_{a b c}^{\prime}=\pi_{1}^{*} \hat{n}_{a b c}\right\},\left\{\Lambda_{a b}^{\prime}=0\right\},\left\{A_{a}^{\prime}=0\right\},\left\{B_{a}^{\prime}=0\right\}\right) .
$$

Since $\hat{\Phi}$ is $M^{6 \mid 8 n}$-trivial, its pullback $\Phi^{\prime}$ is holomorphically trivial on all of $F^{9 \mid 8 n}$. Therefore, there exists a relative degree-2 Deligne cochain relating the degree-2 Deligne cocycle (6.16) to the cocycle

$$
\left(\left\{m_{a b}^{\prime \prime}=\operatorname{id}_{e_{a}}\right\},\left\{n_{a b c}^{\prime \prime}=\operatorname{id}_{\mathrm{id}_{e_{a}}}\right\},\left\{\Lambda_{a b}^{\prime \prime} \neq 0\right\},\left\{A_{a}^{\prime \prime} \neq 0\right\},\left\{B_{a}^{\prime \prime} \neq 0\right\}\right) .
$$

From (5.32), we realise that $\Lambda_{a b}^{\prime \prime}: A_{b}^{\prime \prime}-A_{a}^{\prime \prime} \Rightarrow 0$ and

$$
\Lambda_{a c}^{\prime \prime}=\Lambda_{a b}^{\prime \prime}+\Lambda_{b c}^{\prime \prime} .
$$

Hence, $\left\{\Lambda_{a b}^{\prime \prime}\right\}$ is a representative of an element in the Abelian Čech cohomology group $H^{1}\left(F^{9 \mid 8 n}, \Omega_{\pi_{1}}^{1} \otimes \mathfrak{v}\right)$. This cohomology group, however, vanishes as follows immediately from the arguments presented in $[9,11]$ (see also [12]). Therefore, we have a splitting

$$
\Lambda_{a b}^{\prime \prime}=\Lambda_{a}^{\prime \prime}-\Lambda_{b}^{\prime \prime} \quad \text { with } \quad \Lambda_{a}^{\prime \prime}: A_{a}^{\prime \prime \prime}-A_{a}^{\prime \prime} \Rightarrow 0
$$


where the $A_{a}^{\prime \prime \prime}$ define a globally defined $\mathfrak{w}$-valued relative 1 -form $A_{\pi_{1}}^{\prime \prime \prime} \in H^{0}\left(F^{9 \mid 8 n}, \Omega_{\pi_{1}}^{1} \otimes \mathfrak{w}\right)$, that is, $A_{a}^{\prime \prime \prime}=\left.A_{\pi_{1}}^{\prime \prime \prime}\right|_{U_{a}^{\prime}}$ and $A_{a}^{\prime \prime \prime}=A_{b}^{\prime \prime \prime}$ on $U_{a}^{\prime} \cap U_{b}^{\prime}$. Thus, using (5.35) with $\Lambda_{a}^{\prime \prime}$, we see that the degree-2 Deligne cocycle (6.17) is cohomologous to

$$
\left(\left\{m_{a b}^{\prime \prime \prime}=\operatorname{id}_{e_{a}}\right\},\left\{n_{a b c}^{\prime \prime \prime}=\operatorname{id}_{\operatorname{id}_{e_{a}}}\right\},\left\{\Lambda_{a b}^{\prime \prime \prime}=0\right\},\left\{A_{a}^{\prime \prime \prime} \neq 0\right\},\left\{B_{a}^{\prime \prime \prime} \neq 0\right\}\right),
$$

where the $B_{a}^{\prime \prime \prime}$ yield a globally defined $\mathfrak{v}$-valued relative 2-form $B_{\pi_{1}}^{\prime \prime \prime} \in H^{0}\left(F^{9 \mid 8 n}, \Omega_{\pi_{1}}^{2} \otimes \mathfrak{v}\right)$, that is, $B_{a}^{\prime \prime \prime}=\left.B_{\pi_{1}}^{\prime \prime \prime}\right|_{U_{a}^{\prime}}$ and $B_{a}^{\prime \prime \prime}=B_{b}^{\prime \prime \prime}$ on $U_{a}^{\prime} \cap U_{b}^{\prime}$.

Altogether, we have obtained a holomorphically trivial semistrict principal 2-bundle $\Phi^{\prime}$ on the correspondence space, equipped with a globally defined relative connective structure represented by $\left(A_{\pi_{1}}, B_{\pi_{2}}\right)$. As this relative connective structure is pure gauge, its curvatures necessarily vanish, and, therefore, the relative connective structure is globally flat.

(ii) $\rightarrow$ (i) Conversely, starting from a holomorphically trivial semistrict principal 2bundle $\Phi^{\prime}$ on the correspondence space represented by a relative degree-2 Deligne cocycle of the form (6.20) with a relative connective structure that is flat, we can use a generalised Poincaré lemma [56] to find a relative degree-2 Deligne cochain to transform (6.20) into a cocycle of the form (6.17). This cocycle descends down to twistor space to a relative degree-2 Deligne cocycle of the form (6.16).

Note that there are equivalence transformations acting on the ingredients of this construction. For instance, constructing the degree-2 Deligne cochains explicitly that mediate between the different degree-2 Deligne cocycles amounts to solving Riemann-Hilbert problems whose solutions are not unique. We shall come back to this in Remark 6.6.

Next, we write the relative exterior derivative explicitly as

$$
\mathrm{d}_{\pi_{1}}=e_{A} V^{A}+e_{I A B} V^{I A B}=e_{[A} \lambda_{B]} \partial^{A B}+e_{I}^{A B} \lambda_{A} D_{B}^{I},
$$

thereby introducing the relative 1 -forms $e_{A}$ and $e_{I A B}=\frac{1}{2} \varepsilon_{A B C D} e_{I}^{C D}$ which are defined dually to $V^{A}$ and $V^{I A B}$. Notice that since $\lambda_{A} V^{A}=\lambda_{A} V^{I A B}=0$, these differential 1-forms are defined modulo terms proportional to $\lambda_{A}$; see also $[9,11]$ for more details.

Lemma 6.3. Let $\alpha_{\pi_{1}} \in H^{0}\left(F^{9 \mid 8 n}, \Omega_{\pi_{1}}^{1}\right), \beta_{\pi_{1}} \in H^{0}\left(F^{9 \mid 8 n}, \Omega_{\pi_{1}}^{2}\right)$, and $\gamma_{\pi_{1}} \in H^{0}\left(F^{9 \mid 8 n}, \Omega_{\pi_{1}}^{2}\right)$. These relative differential forms are then expanded in $\lambda_{A}$ according to

$$
\begin{aligned}
\alpha_{\pi_{1}}= & e_{[A} \lambda_{B]} \alpha^{A B}+e_{I}^{A B} \lambda_{A} \alpha_{B}^{I}, \\
\beta_{\pi_{1}}= & -\frac{1}{4} e_{A} \wedge e_{B} \lambda_{C} \varepsilon^{A B C D} \beta_{D}^{E} \lambda_{E}+\frac{1}{2} e_{A} \lambda_{B} \wedge e_{I}^{E F} \lambda_{E} \varepsilon^{A B C D} \beta_{C D F}^{I}+ \\
& +\frac{1}{2} e_{I}^{C A} \lambda_{C} \wedge e_{J}^{D B} \lambda_{D} \beta_{A B}^{I J}, \\
\gamma_{\pi_{1}}= & -\frac{1}{3} e_{A} \wedge e_{B} \wedge e_{C} \lambda_{D} \varepsilon^{A B C D} \gamma^{E F} \lambda_{E} \lambda_{F}+ \\
& -\frac{1}{4} e_{A} \wedge e_{B} \lambda_{C} \varepsilon^{A B C D} \wedge e_{I}^{E F} \lambda_{E}\left(\gamma_{D}^{G I}\right)_{0} \lambda_{G}+ \\
& +\frac{1}{4} e_{A} \lambda_{B} \wedge e_{I}^{E F} \lambda_{E} \wedge e_{J}^{G H} \lambda_{G} \varepsilon^{A B C D}\left(\gamma_{C D}^{I J}\right)_{0}+ \\
& +\frac{1}{6} e_{I}^{D A} \lambda_{D} \wedge e_{J}^{E B} \lambda_{E} \wedge e_{K}^{F C} \lambda_{F} \gamma_{A B C}^{I J K},
\end{aligned}
$$


where the coefficient functions depend only on the superspace coordinates $\left(x^{A B}, \eta_{I}^{A}\right) \in$ $M^{6 \mid 8 n}$. The component $\left(\gamma_{A}^{B I}\right)_{0}$ is the totally trace-less part of $\gamma_{A}^{B I} C$ while $\left(\gamma_{A B}^{I J}\right)_{0}$ denotes the part of $\gamma_{A B}^{I J}$ that vanishes under contraction with $\varepsilon^{A B C D}$.

Proof. This is a direct consequence of the explicit form of direct images of the sheaves $\Omega_{\pi_{1}}^{1}$ and $\Omega_{\pi_{1}}^{2}$ under the projection $\pi_{2}: F^{9 \mid 8 n} \rightarrow M^{6 \mid 8 n}$. See references $[9,11]$ for a detailed derivation.

Remark 6.4. Note that differential 1-, 2- and 3-forms $\alpha, \beta$, and $\gamma$ on chiral superspace $M^{6 \mid 8 n}$ have components

$$
\left(\alpha_{A B}, \alpha_{B}^{I}\right), \quad\left(\beta_{A}^{B}, \beta_{A B C}^{I}, \beta_{A B}^{I J}\right), \quad \text { and } \quad\left(\gamma_{A B}, \gamma^{A B}, \gamma_{A}^{B I}{ }_{C}, \gamma_{A B C D}^{I J}, \gamma_{A B C}^{I J K}\right)
$$

where $\gamma_{A}{ }_{C}^{B I}$ is traceless over the $A B$ indices. By virtue of Lemma 6.3, we realise that all of these components for the 1- and 2-forms and some of these components for the 3-form appear in the expansion of relative 1-, 2- and 3-forms $\alpha_{\pi_{1}}, \beta_{\pi_{1}}$, and $\gamma_{\pi_{1}}$ on the correspondence $F^{9 \mid 8 n}$. Note further that the components $\left(\gamma_{A B}, \gamma^{A B}\right)$ represent the self-dual and anti-self dual parts of a Graßmann-even differential 3-form $\gamma$ on $M^{6 \mid 0}$.

These considerations then enable us to prove the following Penrose-Ward transform.

Theorem 6.5. Consider the double fibration (6.6). There is a bijection between

(i) equivalence classes of topologically trivial $M^{6 \mid 8 n}$-trivial holomorphic semistrict principal 2-bundles on $P^{6 \mid 2 n}$ and

(ii) gauge equivalence classes of (complex holomorphic) solutions to the constraint equations

$$
\mathcal{F}_{A}^{B}=0, \quad \mathcal{F}_{A B C}^{I}=0, \quad \text { and } \quad \mathcal{F}_{A B}^{I J}=0,
$$

and

$$
\begin{aligned}
H^{A B} & =0, \\
H_{A}^{B I} C & =\delta_{C}^{B} \psi_{A}^{I}-\frac{1}{4} \delta_{A}^{B} \psi_{C}^{I}, \\
H_{A B C D}^{I J} & =\varepsilon_{A B C D} \phi^{I J}, \\
H_{A B C}^{I J K} & =0
\end{aligned}
$$

on chiral superspace $M^{6 \mid 8 n}$. Here, the curvatures read explicitly as

$$
\begin{aligned}
\mathcal{F}_{A}^{B} & =\partial^{B C} A_{C A}-\partial_{C A} A^{B C}+\mu_{2}\left(A^{B C}, A_{C A}\right)-\mu_{1}\left(B_{A}{ }^{B}\right) \\
\mathcal{F}_{A B C} I & =\partial_{A B} A_{C}^{I}-D_{C}^{I} A_{A B}+\mu_{2}\left(A_{A B}, A_{C}^{I}\right)-\mu_{1}\left(B_{A B C}\right) \\
\mathcal{F}_{A B}^{I J} & =D_{A}^{I} A_{B}^{J}+D_{B}^{J} A_{A}^{I}+\mu_{2}\left(A_{A}^{I}, A_{B}^{J}\right)+4 \Omega^{I J} A_{A B}-\mu_{1}\left(B_{A B}^{I J}\right)
\end{aligned}
$$


and

$$
\begin{aligned}
& H_{A B}=\nabla_{C(A} B_{B)}{ }^{C}+\mu_{3}\left(A_{C(A}, A^{C D}, A_{B) D}\right), \\
& H^{A B}=\nabla^{C(A} B_{C}{ }^{B)}+\mu_{3}\left(A^{C(A}, A_{C D}, A^{B) D}\right), \\
& H_{A}{ }_{C}{ }_{C}^{I}=\nabla_{C}^{I} B_{A}^{B}-\nabla^{D B} B_{D A C}^{I}+\nabla_{D A} B_{C}^{D B I}-\mu_{3}\left(A_{C}^{I}, A^{B D}, A_{D A}\right) \text {, } \\
& H_{A B C D}^{I J}=\nabla_{A B} B_{C D}^{I J}-\nabla_{C}^{I} B_{A B} \stackrel{J}{D}-\nabla_{D}^{J} B_{A B C}^{I}- \\
& -2 \Omega^{I J}\left(\varepsilon_{A B F[C} B_{D]}^{F}-\varepsilon_{C D F\left[{ }_{A} B_{B]}{ }^{F}\right.}\right)-\mu_{3}\left(A_{A B}, A_{C}^{I}, A_{D}^{J}\right), \\
& H_{A B C}^{I J K}=\nabla_{A}^{I} B_{B C}^{J K}+\nabla_{B}^{J} B_{A C}^{I K}+\nabla_{C}^{K} B_{A B}^{I J}+ \\
& +4 \Omega^{I J} B_{A B C}+4 \Omega^{I K} B_{A C B}^{J}+4 \Omega^{J K} B_{B C A}^{I}-\mu_{3}\left(A_{A}^{I}, A_{B}^{J}, A_{C}^{K}\right) \text {. }
\end{aligned}
$$

Before proving the theorem, let us make a few comments. The fields $\psi_{A}^{I}$ are Graßmannodd spinor fields while the fields $\phi^{I J}$ are Graßmann-even scalar fields. The condition $H^{A B}=0$ implies that the Graßmann-even part of the 3-form $H$ is self-dual, cf. Remark 6.4. Altogether, $\left(H_{A B}, \psi_{A}^{I}, \phi^{I J}\right)$ constitutes an $\mathcal{N}=(n, 0)$ tensor multiplet for $n=0,1,2$. Note that only for $n=2$, the condition $\phi^{I J} \Omega_{I J}=0$ arises, so that we always find the correct number of scalar fields. See also Saemann \& Wolf [9-11] for more details on this point.

Proof of theorem. (i) $\rightarrow$ (ii) By virtue of Proposition 6.2, topologically trivial $M^{6 \mid 8 n}$-trivial holomorphic semistrict principal 2-bundles on twistor space correspond to holomorphically trivial semistrict principal 2-bundles on $F^{9 \mid 8 n}$ equipped with a relative connective structure which is globally flat and vice versa. Therefore, such a bundle on twistor space yields a globally defined relative connective structure $\left(A_{\pi_{1}}, B_{\pi_{1}}\right) \in H^{0}\left(F^{9 \mid 8 n}, \Omega_{\pi_{1}}^{1} \otimes \mathfrak{w}\right) \oplus$ $H^{0}\left(F^{9 \mid 8 n}, \Omega_{\pi_{1}}^{2} \otimes \mathfrak{v}\right)$ on the correspondence space which is flat, that is,

$$
\begin{aligned}
& \mathcal{F}_{\pi_{1}}=\mathrm{d}_{\pi_{1}} A_{\pi_{1}}+\frac{1}{2} \mu_{2}\left(A_{\pi_{1}}, A_{\pi_{1}}\right)-\mu_{1}\left(B_{\pi_{1}}\right)=0, \\
& H_{\pi_{1}}=\mathrm{d}_{\pi_{1}} B_{\pi_{1}}+\mu_{2}\left(A_{\pi_{1}}, B_{\pi_{1}}\right)-\frac{1}{3 !} \mu_{3}\left(A_{\pi_{1}}, A_{\pi_{1}}, A_{\pi_{1}}\right)=0 .
\end{aligned}
$$

Upon using (6.21) and the expansions given in Lemma 6.3, we arrive at the constraint equations (6.24) and (6.25) after a few algebraic manipulations.

(ii) $\rightarrow$ (i) The converse is also readily derived. Given a solution to (6.24) and (6.25), by Lemma 6.3 we can always construct a globally defined relative connective structure $\left(A_{\pi_{1}}, B_{\pi_{1}}\right) \in H^{0}\left(F^{9 \mid 8 n}, \Omega_{\pi_{1}}^{1} \otimes \mathfrak{w}\right) \oplus H^{0}\left(F^{9 \mid 8 n}, \Omega_{\pi_{1}}^{2} \otimes \mathfrak{v}\right)$ on the correspondence space which is flat. This defines a holomorphically trivial semistrict principal 2-bundles on $F^{9 \mid 8 n}$ equipped with a flat relative connective structure. The construction of a topologically trivial $M^{6 \mid 8 n_{-}}$ trivial holomorphic semistrict principal 2-bundles on twistor space then follows directly from Proposition 6.2.

Remark 6.6. Finally, we would like to mention that the gauge transformations of the connective structure $\left(A_{A B}, A_{A}^{I}, B_{A}{ }^{B}, B_{A B}{ }_{C}^{I}, B_{A B}^{I J}\right)$ on $M^{6 \mid 8 n}$ follow directly from the large class of equivalence relations between relative Deligne 2-cocycles of the form (6.20) on $F^{9 \mid 8 n}$. The Deligne 1-cochains parametrising the equivalence relations between relative Deligne 2-cocycles of the form (6.20) are expressed in terms of $p \in H^{0}\left(F^{9 \mid 8 n}, M\right)$ and 
$\Lambda_{\pi_{1}} \in H^{0}\left(F^{9 \mid 8 n}, \Omega_{\pi_{1}}^{1} \otimes \mathfrak{v}\right)$. Their $\lambda_{A^{-} \text {-expansions read as }}$

$$
p=p(x, \eta) \quad \text { and } \quad \Lambda_{\pi_{1}}=e_{[A} \lambda_{B]} \Lambda^{A B}(x, \eta)+e_{I}^{A B} \lambda_{A} \Lambda_{B}^{I}(x, \eta) .
$$

Such Deligne 1-cochains are therefore described by $p(x, \eta), \Lambda_{A B}(x, \eta)$, and $\Lambda_{A}^{I}(x, \eta)$ which themselves form a Deligne 1-cochain encoding an equivalence relation between Deligne 2cocycles on the chiral superspace $M^{6 \mid 8 n}$. The gauge transformations are then simply of the form given in Proposition 5.9.

\section{Acknowledgments}

We are grateful to James Grant for helpful discussions and comments. The work of BJ was supported by grant GAČR P201/12/G028. The work of CS was supported by a Career Acceleration Fellowship from the U.K. Engineering and Physical Sciences Research Council.

\section{A Strong homotopy Lie algebras}

In this appendix, we recall the definitions of strong homotopy Lie algebras and their Chevalley-Eilenberg algebras as well as the homotopy Maurer-Cartan equation together with their infinitesimal gauge symmetries.

Recall that a permutation $\sigma$ of $i+j$ elements is called an $(i, j)$-unshuffle, if the first $i$ and the last $j$ images of $\sigma$ are ordered: $\sigma(1)<\cdots<\sigma(i)$ and $\sigma(i+1)<\cdots<\sigma(i+j)$. Moreover, the graded Koszul sign $\chi\left(\sigma ; x_{1}, \ldots, x_{n}\right)$ of elements $x_{i}$ of a graded vector space is defined via the equation

$$
x_{1} \wedge \cdots \wedge x_{n}=\chi\left(\sigma ; x_{1}, \ldots, x_{n}\right) x_{\sigma(1)} \wedge \cdots \wedge x_{\sigma(n)}
$$

in the free graded algebra $\wedge\left(x_{1}, \ldots, x_{n}\right)$, where $\wedge$ is considered graded antisymmetric.

Definition A.1. [41, 57, 58] An $L_{\infty}$-algebra or strong homotopy Lie algebra is a $\mathbb{Z}$-graded vector space $L=\oplus_{p \in \mathbb{Z}} L_{p}$ endowed with $n$-ary multilinear totally antisymmetric products $\mu_{n}, n \in \mathbb{N}^{*}$, of degree $2-n$, that satisfy the homotopy Jacobi identities

$$
\sum_{i+j=n} \sum_{\sigma} \chi\left(\sigma ; x_{1}, \ldots, x_{n}\right)(-1)^{i \cdot j} \mu_{i+1}\left(\mu_{j}\left(x_{\sigma(1)}, \ldots, x_{\sigma(j)}\right), x_{\sigma(j+1)}, \ldots, x_{\sigma(i+j)}\right)=0
$$

for all $n \in \mathbb{N}^{*}$, where the sum over $\sigma$ is taken over all $(i, j)$-unshuffles.

An alternative sign convention is given in [59], which is obtained from the above one by inverting the signs of all elements of $L$. The homotopy Jacobi identities (A.2) then read as

$$
\sum_{i+j=n} \sum_{\sigma} \chi\left(\sigma ; x_{1}, \ldots, x_{n}\right) \mu_{j+1}\left(\mu_{i}\left(x_{\sigma(1)}, \ldots, x_{\sigma(i)}\right), x_{\sigma(i+1)}, \ldots, x_{\sigma(i+j)}\right)=0 .
$$

A simple example of an $L_{\infty}$-algebra is a differential graded Lie algebra, for which $\mu_{1}$ is the differential, $\mu_{2}$ is the Lie bracket and $\mu_{i}=0$ for $i \geq 3$. Another example of an $L_{\infty}$-algebra is given by the 2 -term $L_{\infty}$-algebras of Definition 2.39 . 
Definition A.2. $A \mathbb{Z}$-graded coalgebra is a $\mathbb{Z}$-graded vector space $L=\oplus_{p \in \mathbb{Z}} L_{p}$ endowed with a coproduct $\Delta: A \rightarrow A \otimes A$ of degree 0 such that $(\mathbb{1} \otimes \Delta) \circ \Delta=(\Delta \otimes \mathbb{1}) \circ \Delta$. A coderivation of degree $k$ on a coalgebra $C$ is a linear map $D: C \rightarrow C$ of degree $k$ such that $\Delta \circ D=(\mathbb{1} \otimes D+D \otimes \mathbb{1}) \circ \Delta$. A differential graded coalgebra is a graded coalgebra endowed with a coderivation $D$ of degree 1 such that $D \circ D=0$.

Each $L_{\infty}$-algebra yields naturally a differential graded coalgebra. We start from an $L_{\infty^{-}}$ algebra $L$, and shift the degree of each element by -1 , arriving at $L[-1]$. The symmetric tensor algebra $\odot{ }^{\bullet}[-1]$ of $L[-1]$ can be regarded as a graded coalgebra with coproduct

$$
\Delta\left(\ell_{1} \odot \cdots \odot \ell_{n}\right):=\sum_{i=0}^{n} \sum_{\sigma}\left(\ell_{\sigma(1)} \otimes \cdots \otimes \ell_{\sigma(i)}\right) \otimes\left(\ell_{\sigma(i+1)} \otimes \cdots \otimes \ell_{\sigma(n)}\right),
$$

where the sum over $\sigma$ is taken over all $(i, n-i)$-unshuffles. Note that on $L[-1]$, the higher products $\mu_{n}$ all have degree 1 and we can add them to a differential $D$, which acts as $\mu_{i}$ on $L[-1]^{\otimes i}$ and on higher tensor powers of $L[-1]$ as a coderivation. The property $D \circ D=0$ is then equivalent to the homotopy Jacobi identities [41].

On the other hand, given a commutative differential graded coalgebra, we can derive a corresponding $L_{\infty}$-algebra. Altogether, we arrive at the following proposition.

Proposition A.3. An $L_{\infty}$-algebra is equivalent to a commutative differential graded coalgebra.

Instead of working with coalgebras, it is usually more convenient to work directly with differential graded algebras. Assuming that the vector subspaces $L_{p} \subseteq L$ are finite dimensional, we can consider the dual complex $L[-1]^{\vee}$ to $L[-1]$.

Definition A.4. The Chevalley-Eilenberg algebra of an $L_{\infty}$-algebra $L$ is the dual of the differential graded coalgebra $\odot^{\bullet} L[-1]$. In particular, $\operatorname{CE}(L):=\odot^{\bullet}\left(L[-1]^{\vee}\right)$ and the differential $\mathrm{d}_{\mathrm{CE}}:=D^{\vee}$ is the dual of the differential $D$ in $\odot \cdot L[-1]$.

It is straightforward to verify the $\mathrm{CE}(L)$ is indeed a differential graded algebra.

The Chevalley-Eilenberg algebra of a Lie algebra $\mathfrak{g}$ is a differential graded algebra that encodes the Lie bracket via the equation

$$
\mathrm{d}_{\mathrm{CE}} \check{\tau}^{k}+\frac{1}{2} f_{i j}^{k} \check{\tau}^{i} \wedge \check{\tau}^{j}=0
$$

where the $\check{\tau}^{i}$ form a basis of the dual $\mathfrak{g}^{\vee}$ of $\mathfrak{g}$ and $f_{i j}^{k}$ are the structure constants of $\mathfrak{g}$ with respect to the dual basis $\left(\tau_{i}\right)$ with $\check{\tau}^{i}\left(\tau_{j}\right)=\delta_{j}^{i}$. Evaluated at an element $a \in \mathfrak{g}[-1]$, we have

$$
\mathrm{d}_{\mathrm{CE}} a+\frac{1}{2}[a, a]=0,
$$

the Maurer-Cartan equation of the differential graded algebra. This equation can be generalised to the case of $L_{\infty}$-algebras. 
Definition A.5. An element $\phi$ of an $L_{\infty}$-algebra is called a homotopy Maurer-Cartan element whenever it satisfies the homotopy Maurer-Cartan equation

$$
\sum_{i=1}^{\infty} \frac{(-1)^{i(i+1) / 2}}{i !} \mu_{i}(\phi, \ldots, \phi)=0 .
$$

Theorem A.6. The homotopy Maurer-Cartan equation is invariant under the following infinitesimal symmetries parameterised by an element $\gamma \in L_{0}$ :

$$
\phi \rightarrow \phi+\delta \phi \quad \text { with } \quad \delta \phi=\sum_{i} \frac{(-1)^{i(i-1) / 2}}{(i-1) !} \mu_{i}(\gamma, \phi, \ldots, \phi) .
$$

Proof. The general proof of this theorem can be found, for instance, in [60]. Here, we give a shortened version for the case $\phi \in L_{1}$, which is the one we are interested in. We start by computing the homotopy Jacobi identities (A.2) for the tuple $(\gamma, \phi, \ldots, \phi)$, obtaining

$$
\begin{aligned}
\sum_{i+j=n} & \left(\begin{array}{c}
n-1 \\
j-1
\end{array}\right)(-1)^{i j} \mu_{i+1}\left(\mu_{j}(\gamma, \phi, \ldots, \phi), \phi, \ldots, \phi\right)+ \\
& +\sum_{i+j=n, i \geq 1}\left(\begin{array}{c}
n-1 \\
j
\end{array}\right)(-1)^{i j+n-1} \mu_{i+1}\left(\mu_{j}(\phi, \ldots, \phi), \phi, \ldots, \phi, \gamma\right)=0
\end{aligned}
$$

or

$$
\begin{aligned}
& \sum_{i+j=n} \frac{1}{(j-1) ! i !}(-1)^{1+i(n-i)-\frac{n}{2}+\frac{n^{2}}{2}} \mu_{i+1}\left(\mu_{j}(\phi, \ldots, \phi, \gamma), \phi, \ldots, \phi\right)+ \\
& \quad+\sum_{i+j=n, i \geq 1} \frac{(-1)^{1+i(n-i)+n-1-\frac{n}{2}+\frac{n^{2}}{2}}}{j !(i-1) !} \mu_{i+1}\left(\mu_{j}(\phi, \ldots, \phi), \phi, \ldots, \phi, \gamma\right)=0 .
\end{aligned}
$$

Next, we note the following identities for $i+j=n$ :

$$
\begin{aligned}
& (-1)^{1+i(n-i)+n-1-\frac{n}{2}+\frac{n^{2}}{2}}=(-1)^{i(n-i)+\frac{n}{2}+\frac{n^{2}}{2}}=(-1)^{i(i+1) / 2+j(j+1) / 2}, \\
& (-1)^{((i+1)(i+2)+j(j-1)) / 2}=(-1)^{1+2 i+i^{2}-\frac{n}{2}-i n+\frac{n^{2}}{2}}=(-1)^{1+i(n-i)-\frac{n}{2}+\frac{n^{2}}{2}} .
\end{aligned}
$$

Now we can compute the variation of (A.7) under the transformations (A.8):

$$
\begin{aligned}
\delta\left(\sum_{i=1}^{\infty} \frac{(-1)^{i(i+1) / 2}}{i !} \mu_{i}(\phi, \ldots, \phi)\right)=\sum_{i=1}^{\infty} \frac{(-1)^{i(i+1) / 2}}{(i-1) !} \mu_{i}(\delta \phi, \ldots, \phi) \\
\quad=\sum_{i=1}^{\infty} \sum_{j=1}^{\infty} \frac{(-1)^{(i(i+1)+j(j-1)) / 2}}{(i-1) !(j-1) !} \mu_{i}\left(\mu_{j}(\gamma, \phi, \ldots, \phi), \phi, \ldots, \phi\right) \\
=\sum_{n=1}^{\infty} \sum_{i+j=n}^{\infty} \frac{(-1)^{((i+1)(i+2)+j(j-1)) / 2}}{i !(j-1) !} \mu_{i+1}\left(\mu_{j}(\gamma, \phi, \ldots, \phi), \phi, \ldots, \phi\right) \\
=-\sum_{n=1}^{\infty} \sum_{i+j=n, i \geq 1} \frac{(-1)^{i(i+1) / 2+j(j+1) / 2}}{j !(i-1) !} \mu_{i+1}\left(\mu_{j}(\phi, \ldots, \phi), \phi, \ldots, \phi, \gamma\right) \\
=-\sum_{i=1}^{\infty} \frac{(-1)^{i(i+1) / 2}}{(i-1) !} \mu_{i+1}\left(\sum_{j=1}^{\infty} \frac{(-1)^{j(j+1) / 2}}{j !} \mu_{j}(\phi, \ldots, \phi), \phi, \ldots, \phi, \gamma\right) \\
=0
\end{aligned}
$$

as a consequence of the homotopy Maurer-Cartan equation (A.7). 


\section{B Groupoid bundles}

In this appendix, we present the parameterisation of a functor from the category of supermanifolds to the category of groupoid bundles with preferred section, completing the discussion of Deligne 1-cocycles with values in a semistrict Lie 2-group. Such cocycles arise from functors between the Cech groupoid and the Lie 2-group (regarded as a monoidal category). Our discussion follows closely the lines of that in section 4, and we shall therefore be concise.

We start from $\mathcal{G}=(M, N)$-valued descent data on surjective submersions $\mathbb{R}^{0 \mid 1} \times X \rightarrow$ $X$, which are represented my $M$-valued 1-cells $\left\{m_{0}:=m\left(\theta_{0}\right)\right\}$ and $N$-valued 2-cells $\left\{n_{01}:=\right.$ $\left.n\left(\theta_{0}, \theta_{1}\right)\right\}$ such that

$$
n_{01}: m_{1} \Rightarrow m_{0} \quad \text { and } \quad n_{01} \circ n_{12}=n_{02} \text {. }
$$

Note that $n_{01}=n_{10}^{-1}$ and we can normalise $m_{0}=m\left(\theta_{0}\right)=\operatorname{id}_{e}+\alpha \theta_{0}$ with $\alpha \in \mathfrak{m}[-1]$. These descent data are trivialised by degree-1 Deligne coboundaries $\left\{n_{0}:=n\left(\theta_{0}\right)\right\}$ with

$$
n_{0}: m_{0} \Rightarrow \operatorname{id}_{e} \text { and } n_{01}=n_{0}^{-1} \circ n_{1} \text {, }
$$

where $n_{0}:=n\left(\theta_{0}\right)=n\left(0, \theta_{0}\right)$. Such a coboundary, and therefore the whole functor under consideration, is parameterised by a $\beta \in \mathfrak{v}=\operatorname{ker}(\mathrm{t}) \subseteq T_{\text {id }_{\text {id }_{e}}} N$ according to

$$
n_{0}=\mathrm{id}_{\mathrm{id}}+\beta \theta_{0},
$$

and we conclude that $m_{0}=\mathrm{id}_{e}+\mathbf{s}(\beta) \theta_{0}$. Equivalence relations on such descent data are described by degree-1 Deligne coboundaries according to

$$
q_{0}: \tilde{m}_{0} \Rightarrow m_{0} \quad \text { and } \quad \tilde{n}_{01}=q_{0}^{-1} \circ n_{01} \circ q_{1}
$$

where

$$
q_{0}=q-\mathrm{d}_{\mathrm{K}} q \theta, \quad q \in N \quad \text { with } \quad \mathrm{s}(q)=\mathrm{t}(q)=\mathrm{id}_{e} .
$$

The trivialising coboundary between $\left(\left\{\tilde{n}_{01}\right\},\left\{\tilde{m}_{0}\right\}\right)$ and the trivial cocycle $\left(\left\{\operatorname{id}_{\mathrm{id}_{e}}\right\},\left\{\mathrm{id}_{e}\right\}\right)$ is then given by the composition

$$
n_{0}^{\prime}:=n_{0} \circ q_{0} .
$$

To compare this coboundary with $n_{0}$, we have to bring it to the form $\tilde{n}_{0}=\tilde{n}\left(0, \theta_{0}\right)$ by a modification transformation. Note that the coboundary relation

$$
\tilde{n}_{01}=q_{0}^{-1} \circ n_{0}^{-1} \circ n_{1} \circ q_{1}=\left(n_{0}^{\prime}\right)^{-1} \circ n_{1}^{\prime}
$$

is invariant under the modification transformation

$$
n_{0}^{\prime} \rightarrow \tilde{n}_{0}=o \circ n_{0}^{\prime}
$$

for some $o \in N$. The modification we need here is given by $o=q^{-1}$. Then

$$
\tilde{n}_{0}=q^{-1} \circ n_{0} \circ q_{0}=q^{-1} \circ\left(\operatorname{id}_{\mathrm{id}_{e}}+\beta \theta\right) \circ\left(q-\mathrm{d}_{\mathrm{K}} q \theta\right)=\mathrm{id}_{\mathrm{id}_{e}}+\tilde{\beta} \theta .
$$


To evaluate the concatenation, we introduce the following linearised forms:

$$
q \circ\left(\mathrm{id}_{\mathrm{id}_{e}}+\rho \theta\right):=q \circ\left(\mathrm{id}_{\mathrm{id}_{e}}+\rho \theta\right) \text { and }\left(\mathrm{id}_{\mathrm{id}_{e}}+\rho \theta\right) \circ q \theta:=\left(\mathrm{id}_{\mathrm{id}_{e}}+\rho \theta\right) \circ q,
$$

which implies

$$
\left(q_{1}+\rho_{1} \theta\right) \circ\left(q_{2}+\rho_{2} \theta\right)=q_{1} \circ q_{2}+\left(\rho_{1} \circ q_{2}\right) \circ\left(q_{1} \circ \rho_{2}\right) \theta .
$$

for all $q, q_{1,2} \in N$ and $\rho, \rho_{1,2} \in \mathfrak{n}[-1]$. With this notation, equation (B.9) simplifies to

$$
\tilde{n}_{0}=\mathrm{id}_{\mathrm{id}_{e}}+\tilde{\beta} \theta=\mathrm{id}_{\mathrm{id}_{e}}+\left(q^{-1} \circ \beta \circ q\right) \circ\left(q^{-1} \circ\left(-\mathrm{d}_{\mathrm{K}} q\right)\right) \theta .
$$

We can now readily read off the cocycle conditions and coboundary relations for the $\left\{B_{a} \in\right.$ $\left.C^{0,1}(\mathfrak{U}, \mathfrak{v})\right\}$ contained in the degree-1 Deligne cochain with values in a semistrict Lie 2group.

Open Access. This article is distributed under the terms of the Creative Commons Attribution License (CC-BY 4.0), which permits any use, distribution and reproduction in any medium, provided the original author(s) and source are credited.

\section{References}

[1] L. Breen and W. Messing, Differential geometry of gerbes, Adv. Math. 198 (2005) 732 [math/0106083] [INSPIRE].

[2] P. Aschieri, L. Cantini and B. Jurčo, Non-Abelian bundle gerbes, their differential geometry and gauge theory, Commun. Math. Phys. 254 (2005) 367 [hep-th/0312154] [INSPIRE].

[3] T. Bartels, Higher gauge theory I: 2-bundles, math/0410328.

[4] J.C. Baez and A.D. Lauda, Higher-dimensional algebra V: 2-groups, Theory Appl. Categ. 12 (2004) 423 , http://www.kurims.kyoto-u.ac.jp/EMIS/journals/TAC/volumes/12/14/12-14.pdf [math/0307200].

[5] J.C. Baez, D. Stevenson, A.S. Crans and U. Schreiber, From loop groups to 2-groups, Homology Homotopy Appl. 9 (2007) 101 [math/0504123] [INSPIRE].

[6] E. Witten, Some comments on string dynamics, in proceedings of Strings '95, Los Angeles U.S.A. (1995) [hep-th/9507121] [InSPIRE].

[7] J. Bagger, N. Lambert, S. Mukhi and C. Papageorgakis, Multiple membranes in M-theory, Phys. Rept. 527 (2013) 1 [arXiv: 1203.3546] [InSPIRE].

[8] D. Fiorenza, H. Sati and U. Schreiber, Multiple M5-branes, string 2-connections and $7 d$ nonabelian Chern-Simons theory, Adv. Theor. Math. Phys. 18 (2014) 229 [arXiv:1201.5277] [INSPIRE].

[9] C. Sämann and M. Wolf, Non-Abelian tensor multiplet equations from twistor space, Commun. Math. Phys. 328 (2014) 527 [arXiv: 1205.3108] [INSPIRE].

[10] C. Säemann and M. Wolf, Six-dimensional superconformal field theories from principal 3-bundles over twistor space, Lett. Math. Phys. 104 (2014) 1147 [arXiv:1305.4870] [INSPIRE]. 
[11] C. Sämann and M. Wolf, On twistors and conformal field theories from six dimensions, J. Math. Phys. 54 (2013) 013507 [arXiv:1111.2539] [INSPIRE].

[12] L.J. Mason, R.A. Reid-Edwards and A. Taghavi-Chabert, Conformal field theories in six-dimensional twistor space, J. Geom. Phys. 62 (2012) 2353 [arXiv:1111.2585] [InSPIRE].

[13] H. Samtleben, E. Sezgin and R. Wimmer, (1,0) superconformal models in six dimensions, JHEP 12 (2011) 062 [arXiv:1108.4060] [INSPIRE].

[14] H. Samtleben, E. Sezgin, R. Wimmer and L. Wulff, New superconformal models in six dimensions: gauge group and representation structure, PoS(CORFU2011) 071 [arXiv:1204.0542] [INSPIRE].

[15] H. Samtleben, E. Sezgin and R. Wimmer, Six-dimensional superconformal couplings of non-abelian tensor and hypermultiplets, JHEP 03 (2013) 068 [arXiv: 1212.5199] [INSPIRE].

[16] I. Bandos, H. Samtleben and D. Sorokin, Duality-symmetric actions for non-Abelian tensor fields, Phys. Rev. D 88 (2013) 025024 [arXiv:1305.1304] [InSPIRE].

[17] S.-L. Ko, D. Sorokin and P. Vanichchapongjaroen, The M5-brane action revisited, JHEP 11 (2013) 072 [arXiv: 1308.2231] [INSPIRE].

[18] C.-S. Chu, A theory of non-Abelian tensor gauge field with non-Abelian gauge symmetry $G \times G$, Nucl. Phys. B 866 (2013) 43 [arXiv: 1108.5131] [InSPIRE].

[19] C.-S. Chu and S.-L. Ko, Non-Abelian action for multiple five-branes with self-dual tensors, JHEP 05 (2012) 028 [arXiv: 1203.4224] [INSPIRE].

[20] S. Palmer and C. Sämann, Six-dimensional $(1,0)$ superconformal models and higher gauge theory, J. Math. Phys. 54 (2013) 113509 [arXiv: 1308.2622] [InSPIRE].

[21] E. Getzler, Lie theory for nilpotent $L_{\infty}$-algebras, Ann. Math. 170 (2009) 271 [math/0404003].

[22] A. Henriques, Integrating $L_{\infty}$-algebras, Comp. Math. 144 (2008) 1017 [math/0603563].

[23] P. Severa, $L_{\infty}$-algebras as 1-jets of simplicial manifolds (and a bit beyond), math/0612349.

[24] R. Zucchini, AKSZ models of semistrict higher gauge theory, JHEP 03 (2013) 014 [arXiv: 1112.2819] [INSPIRE].

[25] Y. Sheng and C. Zhu, Integration of Lie 2-algebras and their morphisms, Lett. Math. Phys. 102 (2012) 223 [arXiv:1109.4002].

[26] S. Palmer and C. Sämann, Self-dual string and higher instanton solutions, Phys. Rev. D 89 (2014) 065036 [arXiv:1312.5644] [INSPIRE].

[27] D. Fiorenza, U. Schreiber and J. Stasheff, Čech cocycles for differential characteristic classes: an $\infty$-Lie theoretic construction, Adv. Theor. Math. Phys. 16 (2012) 149 [arXiv:1011.4735] [INSPIRE].

[28] A. Kotov and T. Strobl, Characteristic classes associated to Q-bundles, Int. J. Geom. Meth. Mod. Phys. 12 (2014) 1550006 [arXiv:0711.4106] [InSPIRE].

[29] J. Bénabou, Lecture Notes in Mathematics. Vol. 4\%: Introduction to bicategories, Springer, Heidelberg Germany (1967).

[30] T. Leinster, Basic bicategories, math/9810017.

[31] T. Leinster, Higher operads, higher categories, Cambridge University Press, Cambridge U.K. (2004). 
[32] G. Kelly, On MacLane's conditions for coherence of natural associativities, commutativities, etc., J. Algebra 1 (1964) 397.

[33] D. Verity, Enriched categories, internal categories and change of base, Reprints Theory Appl. Categ. 20 (2011) 1.

[34] K. Hardie, K. Kamps and R. Kieboom, A homotopy bigroupoid of a topological space, Appl. Categ. Struct. 9 (2001) 311.

[35] S. Mac Lane, Categories for the working mathematician, Graduate Texts in Mathematics, second edition, Springer, Heidelberg Germany (1998).

[36] D. Roytenberg, On weak Lie 2-algebras, in: XXVI Workshop on Geometrical Methods in Physics 2007, P. Kielanowski et al. eds., AIP Conf. Proc. 956 (2007) 180 [arXiv:0712.3461].

[37] J.C. Baez and A.S. Crans, Higher-dimensional algebra VI: Lie 2-algebras, Theory Appl. Categor. 12 (2004) 492 [math/0307263] [INSPIRE].

[38] G. Segal, Classifying spaces and spectral sequences, Publ. Math. IHÉS 34 (1968) 105.

[39] B. Jurčo, From simplicial Lie algebras and hypercrossed complexes to differential graded Lie algebras via 1-jets, J. Geom. Phys. 62 (2012) 2389 [arXiv:1110.0815].

[40] M. Kontsevich, Deformation quantization of Poisson manifolds. I, Lett. Math. Phys. 66 (2003) 157 [q-alg/9709040].

[41] T. Lada and J. Stasheff, Introduction to sh Lie algebras for physicists, Int. J. Theor. Phys. 32 (1993) 1087 [hep-th/9209099] [INSPIRE].

[42] H. Sati, U. Schreiber and J. Stasheff, $L_{\infty}$ algebra connections and applications to String- and Chern-Simons n-transport, in: Quantum Field Theory, B. Fauser, J. Tolksdorf and E. Zeidler eds., Birkhäuser, Boston U.S.A. (2009), pg. 303 [arXiv: 0801 .3480] [INSPIRE].

[43] J.-L. Brylinski, Loop spaces, characteristic classes and geometric quantization, Birkhäuser, Boston U.S.A. (2007).

[44] U. Schreiber and K. Waldorf, Connections on non-Abelian gerbes and their holonomy, Theory Appl. Categ. 28 (2013) 476 [arXiv:0808.1923].

[45] W. Wang, On 3-gauge transformations, 3-curvatures and Gray-categories, J. Math. Phys. 55 (2014) 043506 [arXiv:1311.3796] [INSPIRE].

[46] B. Jurčo, Crossed module bundle gerbes: classification, string group and differential geometry, Int. J. Geom. Meth. Mod. Phys. 8 (2011) 1079 [math/0510078] [INSPIRE].

[47] B. Jurčo, Nonabelian bundle 2-gerbes, arXiv:0911.1552 [INSPIRE].

[48] M.K. Murray, A Penrose transform for the twistor space of an even dimensional conformally flat Riemannian manifold, Ann. Global Anal. Geom. 4 (1986) 71.

[49] L.P. Hughston and W.T. Shaw, Minimal curves in six-dimensions, Class. Quant. Grav. 4 (1987) 869 [INSPIRE].

[50] L.P. Hughston, The wave equation in even dimensions, Twistor Newsletter 9 (1979) 60.

[51] L.P. Hughston, Applications of $\mathrm{SO}(8)$ spinors, in: Gravitation and Geometry: a volume in honour of Ivor Robinson, W. Rindler and A. Trautman eds., Bibliopolis, Naples Italy (1987).

[52] L.P. Hughston and L.J. Mason, A generalized Kerr-Robinson theorem, Class. Quant. Grav. 5 (1988) 275 [INSPIRE]. 
[53] R.J. Baston and M.G. Eastwood, The Penrose transform, Oxford University Press, Oxford U.K. (1990).

[54] L.J. Mason and R.A. Reid-Edwards, The supersymmetric Penrose transform in six dimensions, arXiv:1212.6173 [INSPIRE].

[55] Y.I. Manin, Grundlehren der mathematischen Wissenschaften. Vol. 289: Gauge field theory and complex geometry, Springer, Berlin Germany (1988).

[56] G.A. Demessie and C. Saemann, The Poincaré lemma in categorified differential geometry, work in progress.

[57] T. Lada and M. Markl, Strongly homotopy Lie algebras, Commun. Alg. 23 (1995) 2147 [hep-th/9406095] [INSPIRE].

[58] M. Markl, S. Shnider and J. Stasheff, Operads in algebra, topology and physics, Mathematical Surveys and Monographs, American Mathematical Society Press, Providence U.S.A. (2002).

[59] J. Stasheff, Deformation theory and the Batalin-Vilkovisky master equation, Proceedings of the Conference on Deformation Theory, Ascona Switzerland (1996) [q-alg/9702012] [INSPIRE].

[60] C. Lazaroiu, String field theory and brane superpotentials, JHEP 10 (2001) 018 [hep-th/0107162] [INSPIRE]. 